FIAN/TD/03-13

January 2013

\title{
Operator algebra of free conformal currents via twistors
}

\author{
O.A. Gelfond ${ }^{1}$ and M.A. Vasiliev ${ }^{2}$ \\ ${ }^{1}$ Institute of System Research of Russian Academy of Sciences, \\ Nakhimovsky prospect 36-1, 117218, Moscow, Russia \\ ${ }^{2}$ I.E.Tamm Department of Theoretical Physics, Lebedev Physical Institute, \\ Leninsky prospect 53, 119991, Moscow, Russia
}

\begin{abstract}
Operator algebra of (not necessarily free) higher-spin conformal conserved currents in generalized matrix spaces, that include $3 d$ Minkowski space-time as a particular case, is shown to be determined by an associative algebra $M$ of functions on the twistor space. For free conserved currents, $M$ is the universal enveloping algebra of the higher-spin algebra. Proposed construction greatly simplifies computation and analysis of correlators of conserved currents. Generating function for $n$-point functions of $3 d$ (super)currents of all spins, built from $\mathcal{N}$ free constituent massless scalars and spinors, is obtained in a concise form of certain determinant. Our results agree with and extend earlier bulk computations in the HS $A d S_{4} / C F T_{3}$ framework. Generating function for $n$-point functions of $4 d$ conformal currents is also presented.
\end{abstract}




\section{Contents}

1 Introduction 3

2 Fields, currents and charges 5

2.1 Fields . . . . . . . . . . . . . . . . . . . . 5

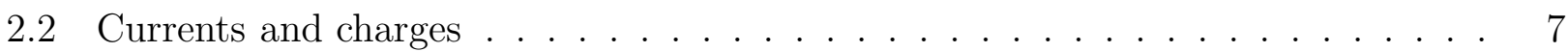

3 D-functions

4 Quantization

5 Algebra of charges

5.1 Charges ........................... . . . 17

5.2 Higher-spin algebra . . . . . . . . . . . . . . . . . . . 20

6 Twistor current operator algebra 21

6.1 OPE of twistor currents . . . . . . . . . . . . . . . . . 22

6.2 Star-product interpretation . . . . . . . . . . . . . . . . 24

6.3 Butterfly algebras . . . . . . . . . . . . . . . . . 25

6.4 Butterfly formulae for multiple products and $n$-point functions . . . . . . . . 27

7 Butterfly formulae from multiparticle algebra $\quad 29$

7.1 Multiple operator products from multiparticle algebra . . . . . . . . . . . 29

7.2 Proof of fundamental relations . . . . . . . . . . . . . . . . 31

8 Space-time current operator algebra 33

8.1 A-currents . . . . . . . . . . . . . . . . . . . 33

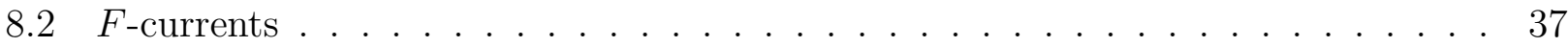

9 Correlators of free conformal currents 38

9.1 Any $M$ and $3 d \ldots \ldots \ldots \ldots$

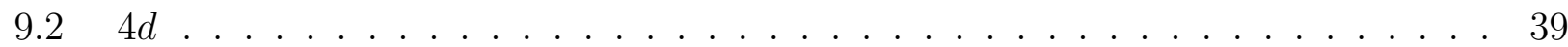

10 Examples 41

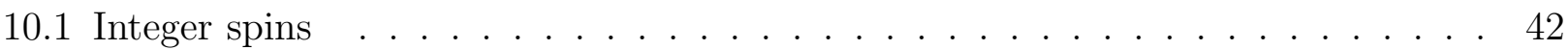

10.2 Half-integer spins . . . . . . . . . . . . . . . . . . . . 43

10.3 Correlators of primary currents of integer spins . . . . . . . . . . . . 44

10.3.1 General case . . . . . . . . . . . . . . . . . . . 44

10.3.2 Two-point function and unitarity . . . . . . . . . . . . . . . . . . . . . . . . . . .

10.3.3 Three-point function . . . . . . . . . . . . . . . . .

11 Conclusion 47

References 


\section{Introduction}

We apply methods of unfolded dynamics to derivation of the operator algebra of conserved currents in three and higher dimensions. The problem is analyzed within the formulation in generalized matrix space $\mathcal{M}_{M}$ that provides a natural framework for description of conformal fields in various space-time dimensions [1, 2, 3, 4, 4, 6, 6]. In the case of $\mathcal{M}_{2}$ equivalent to usual $3 d$ Minkowski space, our analysis reproduces operator algebra of free conformal conserved currents of all spins built from free $3 d$ massless scalars and spinors. We derive full operator algebra of free conserved currents and, in particular, the explicit form of $n$-point functions, including relative coefficients. Obtained results agree with previously available in the literature on two-point, three-point (see, e.g. [7, 8, 9, 10, 11, 12, 13, 14] and references therein) and $n$-point functions [15] for conserved currents of any spin, extending them to supercurrents.

Our construction exhibits covariance under an infinite-dimensional symmetry which extends usual higher-spin (HS) symmetries, that act individually on $n$-point functions with definite $n$, to much larger multiparticle symmetries deduced in [16] from the research of this paper. Mixing states with different number of particles, multiparticle symmetries relate $n$-point functions with different $n$. Being represented by the universal enveloping algebra of the HS algebra, multiparticle algebra $\mathbf{M}$ can be realized [16] in terms of an infinite set of oscillators reminiscent of those of String Theory. Recognition of this symmetry as a promising candidate for the symmetry of a HS generalization of String Theory is an important by-product of our analysis.

The key observation is that, when all spins are involved, conserved currents are described by unrestricted functions $J\left(Y_{i} \mid X\right)$ of the doubled number of spinor (twistor) variables $Y_{i}^{A}$, $i=1,2(A=1, \ldots M)$ [17]. In these terms, free $3 d$ massless fields are described by functions of two-component spinors $y^{\alpha}$ and space-time coordinates $x^{\alpha \beta}=x^{\beta \alpha}$, where $\alpha, \beta=1,2$ are $3 d$

spinor indices, while $3 d$ conserved currents $J\left(y_{i}^{\alpha} \mid x^{\alpha \beta}\right)$ depend on a pair of spinors $y_{1}^{\alpha}, y_{2}^{\alpha}$. Spin $s$ primary HS currents are

$$
J_{\alpha_{1} \ldots \alpha_{2 s}}^{ \pm}(x)=\left.\frac{\partial^{2 s}}{\partial y_{ \pm}^{\alpha_{1}} \ldots \partial y_{ \pm}^{\alpha_{2 s}}} J\left(y_{i}^{\alpha} \mid x\right)\right|_{y=0}, \quad y_{ \pm}^{\alpha}=y_{1}^{\alpha} \pm y_{2}^{\alpha}
$$

OPE of $J\left(y_{i}^{\alpha} \mid x\right) J\left(y_{i}^{\prime \alpha} \mid x^{\prime}\right)$ develops a singularity at $(x, y) \rightarrow\left(x^{\prime}, y^{\prime}\right)$. In particular, for $x=x^{\prime}$, $J\left(y_{i}^{\alpha} \mid x\right) J\left(y_{i}^{\prime \alpha} \mid x\right)$ is a distribution with respect to $y^{\alpha}-y^{\prime \alpha}$. The form of OPE at the same $x$ is very simple in terms of the twistor variables. However, making no sense at $y=y^{\prime}$, such OPE cannot be directly used for the analysis of currents (1.1) of definite spins evaluated at $y=0$. Hence, to compute OPE for currents of definite spins it is necessary to separate their space-time coordinates $x, x^{\prime}$ while for the tower of all spins OPE can be defined at $x=x^{\prime}$.

In more detail, introducing for general $M$,

$$
\mathcal{I}_{g}^{2}=\int_{\mathbb{R}^{2 M}} d^{2 M} Y g\left(Y_{1}, Y_{2}\right) J\left(Y_{1}, Y_{2} \mid 0\right),
$$

where $g\left(Y_{1}, Y_{2}\right)$ is an arbitrary (test) function, operator algebra has the form

$$
\mathcal{I}_{g}^{2} \mathcal{I}_{g^{\prime}}^{2}=: \mathcal{I}_{g}^{2} \mathcal{I}_{g^{\prime}}^{2}:+\mathcal{I}_{\left(g \triangleleft g^{\prime}+g^{\prime} \triangleright g\right)}^{2}+t r_{\triangleright}\left(g \triangleleft g^{\prime}\right) I d,
$$


where $\triangleleft$ and $\triangleright$ denote certain convolution products related to the star product via half-Fourier transform and representing particular cases of certain "butterfly" product law (see Section 6). The first term in (1.3) is regular (terms of this type are usually skipped). The other two are singular. The second term implies that currents generate the HS algebra (see Section 5). The third term is central. The number $\mathcal{N}$ of free fields from which the currents are constructed enters through the definition of $t_{\triangleright}$ which includes usual trace over color indices. Extension of this formula to $\mathcal{I}_{g}^{2}(x) \mathcal{I}_{g^{\prime}}^{2}\left(x^{\prime}\right)$ is uniquely determined by the unfolded current conservation equations, reproducing usual formulas.

Current operator algebra is fully determined in terms of the multiparticle algebra $\mathbf{M}$, leading to the remarkably simple generating function for all $n$-point functions, expressed in terms of certain determinant with respect to butterfly product. This butterfly formula works both for $3 d$ and for $4 d$ Minkowski space. Since all other operators have zero VEV, a $n$-point function coincides with the coefficient in front of the unit operator $I d$ of the operator algebra. As announced in (1.3), such coefficients are expressed in terms of a trace over the butterfly-product algebra. Being similar to the Ansatz of bulk computation in [18, 14, 15], here it results from direct computation which specifies the Green functions involved. Namely, we compute Wightman functions. Of course, at least naively (i.e., away from singularities), from these results it is easy to obtain $T$-ordered correlators by inserting appropriate step-functions in time coordinates.

Our original motivation was to study further $A d S_{4} / C F T_{3}$ HS correspondence that acquired considerable attention after important work of Klebanov and Polyakov [19] where it was argued that the HS gauge theory of [20] should be dual to the $3 d O(\mathcal{N})$ sigma model in the $\mathcal{N} \rightarrow \infty$ limit (for fermionic counterpart see [21]). This conjecture was checked by Giombi and Yin [11] (and references therein) who were able to show in particular how the bulk computation in HS gauge theory reproduces conformal correlators in the free $3 d$ theory. Recently, Maldacena and Zhiboedov [22, 23] addressed the question on restrictions imposed on a boundary $3 d$ conformal theory by HS conformal symmetries. Assuming unitarity and locality they were able to show that a $3 d$ conformal theory, that possesses a HS conserved current, should be free. This conclusion seemingly suggests that any $A d S_{4}$ HS theory should be equivalent to a free boundary theory at least in the most symmetric vacuum. However, in [24] it was shown that, beyond $\mathcal{N} \rightarrow \infty$ limit, holographically dual of the $A d S_{4}$ HS theory is a nonlinear $3 d H S$ theory of conformal currents interactings with $3 d$ conformal HS gauge fields which were not allowed in the analysis of 22, 23].

Results of this paper further support the idea of manifest duality of the bulk $A d S_{4} \mathrm{HS}$ theory and that of boundary currents (plus boundary conformal fields) via unfolded dynamics approach [24]. The appearance of convolution product in the OPE (1.3) is related via a halfFourier transform to the star product underlying minimal HS interactions (see Section 6.2). This fits the standard expectation that the nontrivial part of boundary correlators should result from interactions in the bulk. Moreover, as explained in Sections 2.2 and 11, via replacement of boundary propagators of currents by bulk-to-boundary propagators of bulk fields, the boundary computation of the OPE admits the bulk deformation that does not affect the final result.

From the perspective of a multiparticle HS theory, the computation of $n$-point functions is essentially classical. There are two elements that make the result quantum from usual perspec- 
tive. One is that algebra $\mathbf{M}$ is itself non-commutative as a result of non-commutativity of the HS algebra which is the algebra of oscillators (i.e., Weyl algebra). Another is the origin of the central term proportional to the number $\mathcal{N}$ of free constituent fields in the example of currents of this paper. As discussed in [16], the ambiguity in $\mathcal{N}$ is encoded in the definition of trace on $\mathbf{M}$, which enters the definition of $n$-point function. Hence, both of these quantum effects acquire "classical" interpretation in terms of HS-like computations in multiparticle theory. It is tempting to speculate that such "classical" multiparticle consideration should be equivalent to quantum HS theory with all its multiparticle states involved.

The rest of the paper is organized as follows. In Section 2, we recall relevant facts of the unfolded formulation of fields of different ranks, conserved currents and charges. In Section 3, the construction and properties of $\mathcal{D}$-functions of the unfolded massless field equations are recalled. In Section 4 , quantization prescription for free fields in the generalized matrix space is recalled and quantum currents are introduced. In Section 5, algebra of charges is shown to be isomorphic to the star-product algebra associated with conformal HS algebra. In Section 6, operator algebra of currents in twistor space is introduced and determined for any $M$ in terms of butterfly-product algebra. Derivation of butterfly formulae form the multiparticle algebra of [16] is given in Section 0. Space-time operator algebra is presented in Section 8. General formulae for $3 d$ and $4 d n$-point functions are obtained in Section 9. Numerous examples are considered in Section 10. Conclusions and perspectives are discussed in Section 11 .

\section{Fields, currents and charges}

\section{$2.1 \quad$ Fields}

As shown in [4], conformal fields in various dimensions are conveniently described in terms of generalized space-time $\mathcal{M}_{M}$ with symmetric matrix coordinates $X^{A B}=X^{B A}(A, B=1 \ldots M)$. In the unfolded formulation of [4], conformal fields are formulated in terms of 0 -forms $C^{ \pm}(Y \mid X)$ that depend both on the coordinates $X^{A B}$ and on the spinor (twistor) coordinates $Y^{A}$. Rankone unfolded equations are

$$
\left(\frac{\partial}{\partial X^{A B}} \pm i \frac{\partial^{2}}{\partial Y^{A} \partial Y^{B}}\right) C^{ \pm}(Y \mid X)=0
$$

In the case of $M=2$, these are massless equations for scalar and spinor fields in three space-time dimensions, described, respectively, by even $\left(C^{ \pm}(Y \mid X)=C^{ \pm}(-Y \mid X)\right)$ and odd $\left(C^{ \pm}(Y \mid X)=\right.$ $\left.-C^{ \pm}(-Y \mid X)\right)$ functions of $Y$ [25].

For $M>2$, equations (2.1) are related to conformal equations in higher dimensions as discussed in [2, 3, 4, 5, 6]. In particular, at $M=4$ they describe a tower of $4 d$ conformal (massless) fields of all spins [2, 3, 国. In this section we consider the case of arbitrary $M$, keeping in mind that it is related to the $A d S_{4} / C F T_{3} \mathrm{HS}$ correspondence at $M=2$.

Unfolded formulation [26] is useful in many respects (for more detail and references see [27]). 
In particular, given function $C(Y \mid 0)$ of spinors $Y^{A}$, it reconstructs a solution of Eq. (2.1) by

$$
C^{ \pm}(Y \mid X)=\exp \left(\mp i X^{A B} \frac{\partial^{2}}{\partial Y^{A} \partial Y^{B}}\right) C(Y \mid 0) .
$$

As explained in [28], + and - in Eq. (2.1) distinguish between positive and negative frequencies, i.e., particles and antiparticles. Namely,

$$
C^{ \pm}(Y \mid X)=\frac{1}{(2 \pi)^{\frac{M}{2}}} \int d^{M} \xi c^{ \pm}(\xi) \exp \left[ \pm i\left(\xi_{A} \xi_{B} X^{A B}+Y^{B} \xi_{B}\right)\right]
$$

are complex conjugated to each other

$$
c^{-}(\xi)=\overline{c^{+}(\xi)}, \quad C^{-}(Y \mid X)=\overline{C^{+}(Y \mid X)} .
$$

It is useful to analytically continue $C^{ \pm}(Y \mid X)$ to $C^{ \pm}(\mathcal{Y} \mid \mathcal{X})$ [28] where

$$
\begin{gathered}
\mathcal{X}^{A B}=X^{A B}+i \mathbf{X}^{A B}, \quad X^{A B}=\operatorname{Re} \mathcal{X}^{A B}, \quad \mathbf{X}^{A B}=\operatorname{Im} \mathcal{X}^{A B}, \\
\mathcal{Y}^{A}=Y^{A}+i \mathbf{Y}^{A}, \quad Y^{A}=\operatorname{Re} \mathcal{Y}^{A}, \quad \mathbf{Y}^{A}=\operatorname{Im} \mathcal{Y}^{A} .
\end{gathered}
$$

The real part $X^{A B}$ of $\mathcal{X}^{A B}$ is identified with the coordinates of the generalized space-time containing Minkowski space as a subspace. The imaginary part $\mathbf{X}^{A B}$ is required to be positive definite and was treated in [5] as a regulator that makes the Gaussian integrals well-defined (i.e., physical quantities are obtained in the limit $\mathbf{X}^{A B} \rightarrow 0$; note, that the complex coordinates $Z^{A B}$ of [5] are related to $\mathcal{X}^{A B}$ as $\left.\mathcal{X}^{A B}=i \bar{Z}^{A B}\right) . \mathcal{X}^{A B}$ belong to the upper Siegel half-space $\mathfrak{H}_{M}$ [29, 30]. Evidently, $-\overline{\mathcal{X}}^{A B} \in \mathfrak{H}_{M}$ provided that $\mathcal{X}^{A B} \in \mathfrak{H}_{M}$ and vice versa. The complex variables $\mathcal{Y}^{A}$ extend Siegel space to Fock-Siegel space $\mathfrak{H}_{M} \times \mathbb{C}^{M}$.

Continuation of the functions $C^{ \pm}(2.3)$ to Fock-Siegel space $\mathfrak{H}_{M} \times \mathbb{C}^{M}$ is

$$
\begin{aligned}
C^{+}(\mathcal{Y} \mid \mathcal{X}) & =\frac{1}{(2 \pi)^{\frac{M}{2}}} \int d^{M} \xi c^{+}(\xi) \exp \left[i\left(\xi_{A} \xi_{B} \mathcal{X}^{A B}+\xi_{A} \mathcal{Y}^{A}\right)\right] \\
C^{-}(\overline{\mathcal{Y}} \mid \overline{\mathcal{X}}) & =\frac{1}{(2 \pi)^{\frac{M}{2}}} \int d^{M} \xi c^{-}(\xi) \exp \left[-i\left(\overline{\mathcal{X}}^{A B} \xi_{A} \xi_{B}+\xi_{A} \overline{\mathcal{Y}}^{A}\right)\right]
\end{aligned}
$$

Generalization of the conventional classical field to unfolded dynamics approach is provided by mutually conjugated full fields

$$
\Phi_{j}(Y \mid X)=C_{j}^{+}(Y \mid X)+i^{\mathfrak{p}_{j}} C_{j}^{-}(i Y \mid X), \quad \bar{\Phi}_{j}(Y \mid X)=C_{j}^{-}(Y \mid X)+i^{\mathfrak{p}_{j}} C_{j}^{+}(i Y \mid X),
$$

where $j=1, \ldots, \mathcal{N}$ is the color index, and parity $\mathfrak{p}_{j}=0,1$ distinguishes between bosons and fermions

$$
c_{j}^{ \pm}(-\xi)=(-1)^{\mathfrak{p}_{j}} c_{j}^{ \pm}(\xi) .
$$

Note that, in terms of representations of the $A d S_{4}$ symmetry $s p(4 ; \mathbb{R}), 3 d$ conformal fields correspond to singletons. Hence, adding a singleton is equivalent to the change $\mathcal{N} \rightarrow \mathcal{N}+1$ of 
the number $\mathcal{N}$ of $3 d$ boundary conformal fields, which fact is in agreement with the results of [31] where, however, singleton was described as a bulk field.

Let $\rho$ be an involutive linear map

$$
\begin{gathered}
\rho\left(C_{j}^{ \pm}(Y \mid X)\right)=(i)^{\mathfrak{p}_{j}} C_{j}^{\mp}(i Y \mid X), \\
\rho^{2}\left(C_{j}^{ \pm}(Y \mid X)\right)=(-1)^{\mathfrak{p}_{j}} C_{j}^{ \pm}(-Y \mid X)=C_{j}^{ \pm}(Y \mid X) \quad: \quad \rho^{2}=I d .
\end{gathered}
$$

Then

$$
\Phi_{j}(Y \mid X)=(1+\rho)\left(C_{j}^{+}(Y \mid X)\right), \quad \bar{\Phi}_{j}(Y \mid X)=(1+\rho)\left(C_{j}^{-}(Y \mid X)\right) .
$$

Hence

$$
\rho\left(\Phi_{j}(Y \mid X)\right)=\Phi_{j}(Y \mid X), \quad \rho\left(\bar{\Phi}_{j}(Y \mid X)\right)=\bar{\Phi}_{j}(Y \mid X)
$$

Note that

$$
\Phi_{j}(Y \mid X)=i^{\mathfrak{p}_{j}} \bar{\Phi}_{j}(i Y \mid X), \quad \bar{\Phi}_{j}(Y \mid X)=i^{\mathfrak{p}_{j}} \Phi_{j}(i Y \mid X) .
$$

The factors of $i$ in (2.9) are introduced in such a way that, containing positive and negative frequencies, $\Phi_{j}(Y \mid X)$ and $\Phi_{j}(Y \mid X)$ obey unfolded equations with definite signs of the second terms

$$
\left(\frac{\partial}{\partial X^{A B}}+i \frac{\partial^{2}}{\partial Y^{A} \partial Y^{B}}\right) \Phi_{j}(Y \mid X)=0, \quad\left(\frac{\partial}{\partial X^{A B}}-i \frac{\partial^{2}}{\partial Y^{A} \partial Y^{B}}\right) \Phi_{j}(Y \mid X)=0 .
$$

\subsection{Currents and charges}

Let us recall, following [28], main properties of the unfolded formulation of conserved currents. Rank-two field equation reads as [17]

$$
\left(\frac{\partial}{\partial X^{A B}}-i \frac{\partial^{2}}{\partial U^{(A} \partial V^{B)}}\right) J(U, V \mid X)=0 .
$$

$J(U, V \mid X)$, that satisfies Eq. (2.17), will be called rank-two current field (or, simply, current). Clearly, rank-two fields can be interpreted as bi-local fields in the twistor space. In this respect they are somewhat analogous to space-time bi-local fields sometimes used for the description of currents (see e.g [32, 33] and references therein). The important difference between these two approaches is however that rank-two fields are unconstrained in the twistor space while bilocal space-time fields should obey additional differential conditions with respect to the doubled coordinates. It is this property that makes twistor description efficient.

In unfolded formulation, fundamental dynamical fields, which are primaries in the conformal setup, are described by cohomology of the operator $\sigma_{-}$. For Eq. (2.17), this is the cohomology group $H^{0}\left(\sigma_{-}\right)$where

$$
\sigma_{-}=d X^{A B} \frac{\partial^{2}}{\partial U^{A} \partial V^{B}}
$$

As shown in [17], the primary currents at any $M$ are represented by the following components $j$ of $J(U, V \mid X)$

$$
j_{1}(U \mid X)=J(U, 0 \mid X), \quad j_{2}(V \mid X)=J(0, V \mid X), \quad j_{1,2}=C_{A B}(X) U^{A} V^{B}
$$


where $C_{A B}(X)=-C_{B A}(X)$. All other components of $J(U, V \mid X)$ are expressed via derivatives of the primary currents by unfolded equations (2.17).

Any current $J(U, V \mid X)$ can be decomposed into a sum of currents $J^{h}(U, V \mid X)$ of different "helicities" $h$, that satisfy

$$
H J^{h}(U, V \mid X)=h J^{h}(U, V \mid X),
$$

where the helicity operator

$$
H=\frac{1}{2}\left(V^{A} \frac{\partial}{\partial V^{A}}-U^{A} \frac{\partial}{\partial U^{A}}\right)
$$

commutes to the operator on the l.h.s. of Eq. (2.17), and $h$ is some integer or half-integer.

Since $J(U, V \mid X)$ obeys (2.17), one can use the evolution formula analogous to (2.2)

$$
J(U, V \mid X)=\exp \left(i X^{A B} \frac{\partial^{2}}{\partial V^{A} \partial U^{B}}\right) J(U, V \mid 0) .
$$

A rank-two current field gives rise to the $M$-forms [28]

$$
\Omega(J)=\left.\frac{1}{2}\left(i d X^{A B} \frac{\partial}{\partial U^{B}}+d V^{A}\right)^{M} J(U, V \mid X)\right|_{U=0}
$$

and

$$
\widetilde{\Omega}(J)=\left.\frac{1}{2}\left(i d X^{A B} \frac{\partial}{\partial V^{B}}+d U^{A}\right)^{M} J(U, V \mid X)\right|_{V=0},
$$

that are closed by virtue of (2.17). As a result, on solutions of (2.17), the charges

$$
Q(J)=\int_{\Sigma} \Omega(J), \quad \widetilde{Q}(J)=\int_{\Sigma} \widetilde{\Omega}(J)
$$

are independent of local variations of a $M$-dimensional integration surface $\Sigma \subset \mathcal{M}_{M} \otimes \mathbb{R}^{M}$. For currents that decrease fast enough at space infinity of $\mathcal{M}_{M}$, the charges (2.25) turn out to be independent of the time parameter in $\mathcal{M}_{M}$, thus being conserved.

An important realization of a rank-two current field is provided by the generalized stress tensor constructed from bilinears of free rank-one fields $C_{i}^{ \pm}$in $\mathcal{M}_{M} \otimes \mathbb{R}^{M}$, that satisfy (2.1) and carry color indices $i, j=1,2, \ldots \mathcal{N}$

$$
T_{i j}(U, V \mid X)=C_{i}^{+}(V-U \mid X) C_{j}^{-}(U+V \mid X) .
$$

Eq. (2.17) is equivalent to

$$
\left(\frac{\partial}{\partial X^{A B}}+i \frac{\partial^{2}}{\partial Y_{1}^{A} \partial Y_{1}^{B}}-i \frac{\partial^{2}}{\partial Y_{2}^{A} \partial Y_{2}^{B}}\right) \mathcal{T}_{i j}\left(Y_{1}, Y_{2} \mid X\right)=0,
$$

where

$$
\mathcal{T}_{i j}\left(Y_{1}, Y_{2} \mid X\right)=T_{i j}(U(Y), V(Y) \mid X), \quad V(Y)=\frac{1}{2}\left(Y_{1}+Y_{2}\right), \quad U(Y)=\frac{1}{2}\left(Y_{2}-Y_{1}\right)
$$


The first-order differential operators $\mathcal{A}(Y \mid X)$

$$
\mathcal{A}_{a}{ }^{B}(Y \mid X)=2 i X^{A B} \frac{\partial}{\partial Y^{A}}-a Y^{B}, \quad \mathcal{A}_{a C}(Y \mid X)=\frac{\partial}{\partial Y^{C}}, \quad a=+,-
$$

which have the property

$$
\mathcal{A}_{ \pm}(-i Y \mid X)=i \mathcal{A}_{\mp}(Y \mid X)
$$

obey

$$
\left[\frac{\partial}{\partial X^{A B}} \pm i \frac{\partial^{2}}{\partial Y^{A} \partial Y^{B}}, \mathcal{A}_{ \pm}(Y \mid X)\right]=0
$$

Hence, any polynomial of these operators $\eta\left(\mathcal{A}_{+}\left(Y_{1} \mid X\right), \mathcal{A}_{-}\left(Y_{2} \mid X\right)\right)$ obeys

$$
\left[\left(\frac{\partial}{\partial X^{A B}}+i \frac{\partial^{2}}{\partial Y_{1}^{A} \partial Y_{1}^{B}}-i \frac{\partial^{2}}{\partial Y_{2}^{A} \partial Y_{2}^{B}}\right), \eta\left(\mathcal{A}_{+}\left(Y_{1} \mid X\right), \mathcal{A}_{-}\left(Y_{2} \mid X\right)\right)\right]=0 \text {. }
$$

Therefore,

$$
J_{\eta}(Y \mid X)=\eta^{i j}\left(\mathcal{A}_{+}\left(Y_{1} \mid X\right), \mathcal{A}_{-}\left(Y_{2} \mid X\right)\right) \mathcal{T}_{i j}\left(Y_{1}, Y_{2} \mid X\right)
$$

obeys the current equation (2.17) with the identification (2.28).

Most of polynomials $\eta^{i j}(\mathcal{A})$ lead to exact forms $(2.23)$ and (2.24) and, hence, zero charges (2.25). Indeed, consider the following operators

$$
\begin{aligned}
\mathcal{B}_{A}(U, V \mid X) & =\frac{\partial}{\partial U^{A}}=-\mathcal{A}_{+A}\left(Y_{1} \mid X\right)+\mathcal{A}_{-A}\left(Y_{2} \mid X\right) \\
\mathcal{B}^{B}(U, V \mid X) & =i X^{A B} \frac{\partial}{\partial U^{A}}+V^{B}=\frac{1}{2}\left(-\mathcal{A}_{+}{ }^{B}\left(Y_{1} \mid X\right)+\mathcal{A}_{-}{ }^{B}\left(Y_{2} \mid X\right)\right) \\
\widetilde{\mathcal{B}}_{A}(U, V \mid X) & =\frac{\partial}{\partial V^{A}}=\mathcal{A}_{+A}\left(Y_{1} \mid X\right)+\mathcal{A}_{-A}\left(Y_{2} \mid X\right) \\
\widetilde{\mathcal{B}}^{B}(U, V \mid X) & =i X^{A B} \frac{\partial}{\partial V^{A}}+U^{B}=\frac{1}{2}\left(\mathcal{A}_{+}{ }^{B}\left(Y_{1} \mid X\right)+\mathcal{A}_{-}{ }^{B}\left(Y_{2} \mid X\right)\right)
\end{aligned}
$$

which obey Heisenberg commutation relations

$$
\left[\widetilde{\mathcal{B}}_{B}(U, V \mid X), \mathcal{B}^{C}(U, V \mid X)\right]=\delta_{B}^{C}, \quad\left[\mathcal{B}_{B}(U, V \mid X), \widetilde{\mathcal{B}}^{C}(U, V \mid X)\right]=\delta_{B}^{C}
$$

As shown in [34, the form $\Omega\left(J_{\eta}\right)$ (2.23) is exact provided that

$$
\eta(\mathcal{B}, \widetilde{\mathcal{B}})=\widetilde{\mathcal{B}}^{B}(U, V \mid X) \eta_{B}(\mathcal{B}, \widetilde{\mathcal{B}})+\widetilde{\mathcal{B}}_{A}(U, V \mid X) \eta^{A}(\mathcal{B}, \widetilde{\mathcal{B}})
$$

for some $\eta_{A}$ and $\eta^{A}$. Analogously, $\eta(\mathcal{B}, \widetilde{\mathcal{B}})$ of the form

$$
\eta(\mathcal{B}, \widetilde{\mathcal{B}})=\mathcal{B}^{B}(U, V \mid X) \eta_{B}(\mathcal{B}, \widetilde{\mathcal{B}})+\mathcal{B}_{A}(U, V \mid X) \eta^{A}(\mathcal{B}, \widetilde{\mathcal{B}})
$$


leads to the exact form $\widetilde{\Omega}\left(J_{\eta}\right)$ (2.24). As a result, nonzero charges $Q$ and $\widetilde{Q}$ are represented, respectively, by $\widetilde{\mathcal{B}}$-independent $\eta(\mathcal{B}(U, V \mid X))$ and $\mathcal{B}$-independent $\eta(\widetilde{\mathcal{B}}(U, V \mid X))$ which can be interpreted as parameters of global HS symmetry transformations generated by the charges

$$
Q\left(J_{\eta}\right)=Q\left(J_{\eta(\mathcal{B})}\right), \quad \widetilde{Q}\left(J_{\eta}\right)=\widetilde{Q}\left(J_{\eta(\widetilde{\mathcal{B}})}\right) .
$$

Since

$$
H(\mathcal{B})=-\frac{1}{2} \mathcal{B}, \quad H(\widetilde{\mathcal{B}})=\frac{1}{2} \widetilde{\mathcal{B}},
$$

where $H$ is the helicity operator (2.21), the charges $\widetilde{Q}$ and $Q$ are supported by the parameters of non-negative and non-positive helicities, respectively. As shown in Section 0 , the charges $\widetilde{Q}$ and $Q$ generate the $N=2$ supersymmetric HS algebra where the number of charges of a given spin is doubled.

Consider involutive maps $P_{1}, P_{2}\left(\left(P_{i}\right)^{2}=1\right)$

$$
P_{1}\left(A\left(Y_{1}, Y_{2}\right)\right)=A\left(-Y_{1}, Y_{2}\right), \quad P_{2}\left(A\left(Y_{1}, Y_{2}\right)\right)=A\left(Y_{1},-Y_{2}\right) .
$$

Equivalently, in terms of $U, V$,

$$
P_{1}(\mathcal{B}(V, U))=\mathcal{B}(U, V), \quad P_{2}(\mathcal{B}(V, U))=\mathcal{B}(-U,-V) .
$$

Since from Eqs. (2.34), (2.35) it follows that $\widetilde{\mathcal{B}} \Leftrightarrow \mathcal{B}$ when $U \Leftrightarrow V$, we observe that

$$
\begin{array}{cl}
P_{1}(\mathcal{B}(U, V \mid X))=\widetilde{\mathcal{B}}(U, V \mid X), & P_{1}(\widetilde{\mathcal{B}}(U, V \mid X))=\mathcal{B}(U, V \mid X), \\
P_{2}(\mathcal{B}(U, V \mid X))=\widetilde{\mathcal{B}}(-U,-V \mid X), & P_{2}(\widetilde{\mathcal{B}}(U, V \mid X))=\mathcal{B}(-U,-V \mid X) .
\end{array}
$$

For the bilinear stress tensor $T_{i j}(2.26)$ this gives

$$
\widetilde{T}_{i j}(U, V \mid X):=P_{1}\left(T_{i j}(U, V \mid X)\right)=C_{i}^{+}(-V+U \mid X) C_{j}^{-}(U+V \mid X) .
$$

Since $C_{i}^{+}(-Y \mid X)$ solves (2.1) provided that $C_{i}^{+}(Y \mid X)$ does, $\widetilde{T}_{i j}(U, V \mid X)$ is also a bilinear stress tensor (2.26). Hence

$$
P_{1}\left(\Omega\left(J_{\eta(\mathcal{B})}\right)\right)=\widetilde{\Omega}\left(\widetilde{J}_{\eta(\widetilde{\mathcal{B}})}\right), \quad \widetilde{J}_{\eta(\widetilde{\mathcal{B}})}=\eta^{i j}(\widetilde{\mathcal{B}}) \widetilde{T}_{i j}(U, V \mid X)
$$

and

$$
Q\left(J_{\eta(\mathcal{B})}\right)=\widetilde{Q}\left(\widetilde{J}_{\eta(\widetilde{\mathcal{B}})}\right) .
$$

The construction of currents admits several generalizations. In particular, rank- $2 r$ currents considered in [28] obey

$$
\left(\frac{\partial}{\partial X^{A B}}-i \sum_{j}^{r} \frac{\partial^{2}}{\partial U_{j}^{A} \partial V_{j}^{B}}\right) J^{2 r}(U, V \mid X)=0 .
$$


A multilinear "stress tensor" is

$$
T^{2 r}(U, V \mid X)=\prod_{j=1}^{r} C_{i_{j}}^{+}\left(V_{j}-U_{j} \mid X\right) C_{k_{j}}^{-}\left(U_{j}+V_{j} \mid X\right),
$$

where $C_{m}^{ \pm}(Y \mid X)$ are solutions of positive- or negative-frequency rank-one equations. Then

$$
J^{2 r}(U, V \mid X)=\eta(\mathcal{B}, \widetilde{\mathcal{B}}) T^{2 r}(U, V \mid X)
$$

where $\eta(\mathcal{B}, \widetilde{\mathcal{B}})$ are polynomial parameters that depend on $\mathcal{B}_{j}(2.34)$ and $\widetilde{\mathcal{B}_{j}}(2.35)$ with $U \rightarrow U_{j}$ and $V \rightarrow V_{j}$, solves (2.46). The multilinear stress tensor (2.47) is nothing but the bilinear stress tensor (2.26) built from the rank-one solutions in $\mathcal{M}_{r M}$, that result from rank- $r$ solutions in $\mathcal{M}_{M}$ realized by the $r$-linear products of rank-one solutions in $\mathcal{M}_{M}$.

Alternatively, one can consider multilinear currents of the form

$$
\mathcal{J}_{\eta_{1} \ldots \eta_{n}}^{2 r}\left(Y_{1}, \ldots, Y_{2 r} \mid X_{1}, \ldots, X_{r}\right)=\prod_{j} \mathcal{J}_{\eta_{j}}\left(Y_{2 j-1}, Y_{2 j} \mid X_{j}\right)
$$

where $\eta_{j}=\eta_{j}\left(\mathcal{A}\left(Y_{2 j-1}, Y_{2 j} \mid X_{j}\right)\right)$ with $\mathcal{A}(2.29)$.

It is important to note that unfolded equation (2.46) as well as its particular cases of ranks one (2.1) and two (2.17) have a form of covariant constancy conditions

$$
D_{0} \mathcal{C}=0, \quad D_{0}=d+\omega_{0},
$$

where $\mathcal{C}$ is any of the fields $C, \Phi$ or $J$ and $D_{0}$ is the covariant derivative of the conformal algebra $s p(4)$ for $M=2$ or its extension $s p(2 M)$ for higher $M$ (see e.g. 画). To describe a conformally flat space-time, the connection $\omega_{0}$ is required to be flat

$$
D_{0}^{2}=0 \text {. }
$$

The equations considered in this paper represent (2.50) in the particular case of $\omega_{0}$ corresponding to Cartesian coordinate system where the only nonzero component is vielbein associated with the translations in the conformal group. However, one can equally well describe the same systems in any other conformally invariant coordinates associated with different $\omega_{0}$.

Moreover, in accordance with the general analysis of [16], this allows one immediately extend consideration to a larger space exhibiting the same symmetry simply by adding additional coordinates. The particularly important case is the extension of $d$-dimensional Minkowski space to $A d S_{d+1}$. (For the constructive definition of the respective connections see [16].) As discussed in some more detail in the preamble of Section 6, this simple observation makes the correspondence between boundary and bulk computations nearly tautological.

\section{D-functions}

$\mathcal{D}$-functions of the massless Klein-Gordon-like equation

$$
\left(\frac{\partial^{2}}{\partial X^{A B} \partial X^{C D}}-\frac{\partial^{2}}{\partial X^{A C} \partial X^{B D}}\right) b(X)=0
$$


and the Dirac-like equation

$$
\frac{\partial}{\partial X^{A B}} f_{C}(X)-\frac{\partial}{\partial X^{A C}} f_{B}(X)=0
$$

in $\mathcal{M}_{M}$ were introduced in [5] as their singular solutions resulting from the integral representation (2.3)

$$
\mathcal{D}^{ \pm}(X)=\mp i(2 \pi)^{-M} \int d^{M} \xi \exp \left[ \pm i\left(\xi_{A} \xi_{B} X^{A B}\right)\right] .
$$

The $Y$ dependence of $\mathcal{D}$-functions reconstructed in [28] via the unfolded equations (2.1) is

$$
\mathcal{D}^{ \pm}(Y \mid X)=\mp i(2 \pi)^{-M} \int d^{M} \xi \exp \left[ \pm i\left(\xi_{A} \xi_{B} X^{A B}+\xi_{A} Y^{A}\right)\right] .
$$

Note that normalization of $\mathcal{D}^{ \pm}$(3.3), (3.4) differs from that of [5, 28] by the factor of $2^{-M}$ to achieve

$$
\left.\mathcal{D}^{ \pm}(Y \mid X)\right|_{X \rightarrow 0}=\mp i \delta^{M}(Y) .
$$

Hence, $\mathcal{D}^{ \pm}(Y \mid X)$ (3.4) belong to the class of solutions of (2.1), that includes distributions in $Y$.

Recall that $\mathcal{D}$-functions have to be distinguished from Green functions. The former solve homogeneous field equations with $\delta$-functional initial data while the latter solve inhomogeneous field equations with the $\delta$-functional right-hand-side. Nevertheless they have similar form away from singularity. As discussed in [5], the difference is essentially due to step-functions in time. As a result, away from singularity, computation of chronologically ordered functions with causal propagators gives the same result as Wightman functions with appropriately ordered arguments. In this paper we compute Wightman functions based on $\mathcal{D}$-functions.

Continuation of $\mathcal{D}^{+}$to Fock-Siegel space

$$
\mathcal{D}^{+}(\mathcal{Y} \mid \mathcal{X})=-i(2 \pi)^{-M} \int d^{M} \xi \exp \left[i\left(\mathcal{X}^{A B} \xi_{A} \xi_{B}+\mathcal{Y}^{A} \xi_{A}\right)\right],
$$

gives

$$
\mathcal{D}^{+}(\mathcal{Y} \mid \mathcal{X})=\frac{-i}{2^{M} \pi^{M / 2}} s^{-1} \exp \left(-\frac{i}{4} \mathcal{X}_{A B}^{-1} \mathcal{Y}^{A} \mathcal{Y}^{B}\right), \quad \mathcal{X}_{A B}^{-1} \mathcal{X}^{B C}=\delta_{A}^{C},
$$

where

$$
s^{2}(\mathcal{X})=\operatorname{det}(-i \mathcal{X})
$$

Evidently,

$$
\mathcal{D}^{-}(\mathcal{Y} \mid \mathcal{X})=\overline{\mathcal{D}}^{+}(\overline{\mathcal{Y}} \mid \overline{\mathcal{X}})
$$

As shown in [5], for non-degenerate $X^{A B}$

$$
\mathcal{D}^{ \pm}(X)=\left.\mp \frac{i}{2^{M} \pi^{\frac{M}{2}}} \exp \left( \pm \frac{i \pi I_{X}}{4}\right) \frac{1}{\sqrt{|\operatorname{det}(\mathcal{X})|}}\right|_{\operatorname{Im} \mathcal{X} \rightarrow 0}
$$


where

$$
I_{X}=n_{+}-n_{-}
$$

is the inertia index of the matrix $X^{A B}$ with $n_{+}$and $n_{-}$being, respectively, the numbers of positive and negative eigenvalues of $X^{A B}$. Integral representation (3.6) provides definition of the regularized expression in the complex space.

From (3.7) and (3.10) it follows that

$$
\begin{gathered}
\mathcal{D}^{ \pm}(Y \mid X)=\mathcal{D}^{ \pm}(X) \exp \left(\mp \frac{i}{4} X_{A B}^{-1} Y^{A} Y^{B}\right)= \\
\left.\mp \frac{i}{2^{M} \pi^{\frac{M}{2}}} \exp \left( \pm \frac{i \pi I_{X}}{4}\right) \frac{1}{\sqrt{|\operatorname{det}(\mathcal{X})|}} \exp \left(\mp \frac{i}{4} \mathcal{X}_{A B}^{-1} Y^{A} Y^{B}\right)\right|_{\operatorname{Im} \mathcal{X} \rightarrow 0} .
\end{gathered}
$$

$\mathcal{D}$-functions provide integral representation for solutions of field equations. The simplest way to see this is to observe that $\mathcal{D}^{ \pm}\left(Y^{\prime}-Y \mid X^{\prime}-X\right)$ solves (2.1) with respect to both $X^{\prime}, Y^{\prime}$ and $X, Y$. Hence, with respect to primed variables,

$$
\mathcal{D}^{ \pm}\left(V^{\prime} \mp U^{\prime}-Y \mid X^{\prime}-X\right) C^{\mp}\left(V^{\prime} \pm U^{\prime} \mid X^{\prime}\right)
$$

is a bilinear stress tensor of the form (2.26) that depends on $Y$ and $X$ as parameters. The respective (spin one) conserved charge gives a solution $C^{\mp}(Y \mid X)$ of (2.1) independent of the choice of integration surface and reproducing $C^{\mp}\left(Y^{\prime} \mid X^{\prime}\right)$ at $X=X^{\prime}, Y=Y^{\prime}$ due to (3.5).

In this paper, we will use the evolution formulae in the space of $Y$ variables [28], which in the limit $\operatorname{Im} \mathcal{X} \rightarrow 0$ can be conveniently written as

$$
C^{a}(Y \mid X)=i \int d^{M} Y^{\prime} \mathcal{D}_{a}\left(Y^{\prime}-Y \mid X^{\prime}-X\right) C^{a}\left(Y^{\prime} \mid X^{\prime}\right) \quad \text { no summation over } a=+,-,
$$

where

$$
\mathcal{D}_{a}(Y \mid X)=\varepsilon_{a b} \mathcal{D}^{b}(Y \mid X), \quad \varepsilon_{-+}=-\varepsilon_{+-}=1 .
$$

The following simple consequence of (3.14) plays the key role in the analysis of Section 8

$$
\int d^{M} p \mathcal{D}_{-}(p-Y \mid X) \mathcal{D}_{+}\left(p-Y^{\prime} \mid X^{\prime}\right)=-i \mathcal{D}_{-}\left(Y-Y^{\prime} \mid X-X^{\prime}\right)=-i \mathcal{D}_{+}\left(Y-Y^{\prime} \mid X^{\prime}-X\right) .
$$

Away from singularities, i.e., at $\operatorname{det} X \neq 0$, from Eqs. (3.12) it follows that

$$
\begin{gathered}
\mathcal{D}_{ \pm}(Y \mid X)=\mathcal{D}_{ \pm}(X) \exp \left( \pm \frac{i}{4} X_{A B}^{-1} Y^{A} Y^{B}\right), \quad \mathcal{D}_{+}(Y \mid X)=\mathcal{D}_{-}(Y \mid-X), \\
\mathcal{D}_{-}(i Y \mid-X)=\mathcal{D}_{+}(i Y \mid X)=\exp \left(\frac{i \pi I_{X}}{2}\right) \mathcal{D}_{-}(Y \mid X)=\exp \left(\frac{i \pi I_{X}}{2}\right) \mathcal{D}_{+}(Y \mid-X) .
\end{gathered}
$$

Formula (3.14), providing at fixed $X^{\prime}$ the map from the twistor space to (boundary) $X$ space, allows us to call $\mathcal{D}_{a}$ twistor-to-boundary $\mathcal{D}$-functions. In accordance with the discussion in the end of Section 2.2, their extension to twistor-to-bulk $\mathcal{D}$-functions with the same initial data (3.5) provides an extension of the whole setup to the bulk. 


\section{Quantization}

As usual, the decomposition into positive- and negative-frequency parts (2.3) gives rise to the quantum creation and annihilation operators [0] $\hat{\mathfrak{c}}_{j}^{ \pm}(\xi)$ ( $j$ is color index). The (anti)commutation relations are [5]

$$
\left[\hat{\mathfrak{c}}_{j}^{-}\left(\xi_{1}\right), \hat{\mathfrak{c}}_{k}^{+}\left(\xi_{2}\right)\right]_{ \pm}=\delta_{j k} \frac{1}{2}\left(\delta^{M}\left(\xi_{1}-\xi_{2}\right)+(-1)^{\mathfrak{p}_{j} \mathfrak{p}_{k}} \delta^{M}\left(\xi_{1}+\xi_{2}\right)\right), \quad\left[\hat{\mathfrak{c}}_{j}^{ \pm}\left(\xi_{1}\right), \hat{\mathfrak{c}}_{k}^{ \pm}\left(\xi_{2}\right)\right]_{ \pm}=0
$$

where $\mathfrak{p}_{j}=0,1$ is the boson-fermion parity (2.10). For quantum fields

$$
\hat{C}_{j}^{ \pm}(Y \mid X)=(2 \pi)^{-\frac{M}{2}} \int d^{M} \xi \hat{\mathfrak{c}}_{j}^{ \pm}(\xi) \exp \left[ \pm i\left(\xi_{A} \xi_{B} X^{A B}+\xi_{A} Y^{A}\right)\right]
$$

this gives

$\left[\hat{C}_{j}^{-}(Y \mid X), \hat{C}_{k}^{+}\left(Y^{\prime} \mid X^{\prime}\right)\right]_{ \pm}=\frac{1}{2 i} \delta_{j k}\left\{\mathcal{D}^{-}\left(Y-Y^{\prime} \mid X-X^{\prime}\right)+(-1)^{\mathfrak{p}_{j} \mathfrak{p}_{k}} \mathcal{D}^{-}\left(Y+Y^{\prime} \mid X-X^{\prime}\right)\right\}$,

with $\mathcal{D}^{-}$(3.4). By virtue of (3.5), at $X=X^{\prime}$ this gives

$$
\left[\hat{C}_{j}^{-}(Y \mid X), \hat{C}_{k}^{+}\left(Y^{\prime} \mid X\right)\right]_{ \pm}=\frac{1}{2} \delta_{j k}\left(\delta\left(Y-Y^{\prime}\right)+(-1)^{\mathfrak{p}_{j} \mathfrak{p}_{k}} \delta\left(Y+Y^{\prime}\right)\right)
$$

Generalized quantum HS stress tensor is

$$
\hat{\mathcal{T}}_{j k}\left(Y_{1}, Y_{2} \mid X\right)=: \hat{\Phi}_{j}\left(Y_{1} \mid X\right) \hat{\bar{\Phi}}_{k}\left(Y_{2} \mid X\right):
$$

with the quantized full fields (2.9) $\hat{\Phi}_{j}, \hat{\bar{\Phi}}_{j}$. As usual, normal ordering sends $\hat{C}^{+}$to the left and $\hat{C}^{-}$to the right, i.e.,

$$
: \hat{C}_{k}^{-}\left(Y_{2} \mid X\right) \hat{C}_{j}^{+}\left(Y_{1} \mid X\right):=(-1)^{\mathfrak{p}_{j} \mathfrak{p}_{k}} \hat{C}_{j}^{+}\left(Y_{1} \mid X\right) \hat{C}_{k}^{-}\left(Y_{2} \mid X\right) .
$$

Substitution of (2.9) into (4.5) gives

$$
\hat{\mathcal{T}}_{j k}\left(Y_{1}, Y_{2} \mid X\right)=\sum_{a, b=+,-}\left(\kappa_{1}^{a}\right)^{\mathfrak{p}_{j}}\left(\kappa_{2}^{b}\right)^{\mathfrak{p}_{k}} \hat{T}_{j k}^{a b}\left(\kappa_{1}^{a} Y_{1}, \kappa_{2}^{b} Y_{2} \mid X\right),
$$

where

$$
\hat{\mathrm{T}}_{j k}^{a b}\left(W_{1}, W_{2} \mid X\right)=: \hat{C}_{j}^{a}\left(W_{1} \mid X\right) \hat{C}_{k}^{b}\left(W_{2} \mid X\right):
$$

and

$$
\kappa_{1}^{+}=\kappa_{2}^{-}=1, \quad \kappa_{2}^{+}=\kappa_{1}^{-}=i
$$

Note that

$$
\begin{aligned}
& \hat{\mathrm{T}}_{j k}^{a b}\left(W_{1}, W_{2} \mid X\right)=(-1)^{\mathfrak{p}_{j} \mathfrak{p}_{k}} \hat{\mathrm{T}}_{k j}^{b a}\left(W_{2}, W_{1} \mid X\right), \\
& \hat{\mathrm{T}}_{j k}^{a b}\left(W_{1}, W_{2} \mid X\right)=(-1)^{\mathfrak{p}_{j}} \hat{\mathrm{T}}_{j k}^{a b}\left(-W_{1}, W_{2} \mid X\right)=(-1)^{\mathfrak{p}_{k}} \hat{\mathrm{T}}_{j k}^{a b}\left(W_{1},-W_{2} \mid X\right)
\end{aligned}
$$


and

$$
\left(\kappa_{1}^{a}\right)^{-1}=a \kappa_{1}^{a}, \quad\left(\kappa_{2}^{a}\right)^{-1}=-a \kappa_{2}^{a}, \quad i \kappa_{2}^{a}=-a \kappa_{1}^{a} .
$$

Let us extend the action of $\rho$ (2.11) to $\hat{\mathcal{T}}_{j k}\left(Y_{1}, Y_{2} \mid X\right)$. Assuming that $\rho$ is an antiautomorphism and using (2.14) we obtain

$$
\rho\left(\hat{\mathcal{T}}_{j k}\left(Y_{1}, Y_{2} \mid X\right)\right)=: \rho\left(\hat{\bar{\Phi}}_{k}\left(Y_{2} \mid X\right)\right) \rho\left(\hat{\Phi}_{j}\left(Y_{1} \mid X\right)\right):=(-1)^{\mathfrak{p}_{k} \mathfrak{p}_{j}} \hat{\mathcal{T}}_{j k}\left(Y_{1}, Y_{2} \mid X\right) .
$$

Also, by virtue of (2.15) along with

$$
i^{\mathfrak{p}_{j, k}}=i^{\mathfrak{p}_{j}+\mathfrak{p}_{k}}(-1)^{\mathfrak{p}_{k} \mathfrak{p}_{j}}, \quad \mathfrak{p}_{j, k}=\left(\mathfrak{p}_{j}+\mathfrak{p}_{k}\right) \quad \bmod 2 \quad(\{p \bmod 2\}=0 \text { or } 1),
$$

where $\mathfrak{p}_{j, k}$ is the boson-fermion parity of $\hat{\mathcal{T}}_{j k}\left(Y_{1}, Y_{2} \mid X\right)$,

$$
\hat{\mathcal{T}}_{j k}\left(Y_{1}, Y_{2} \mid X\right)=i^{\mathfrak{p}_{j, k}} \hat{\mathcal{T}}_{k j}\left(i Y_{2}, i Y_{1} \mid X\right) .
$$

From

$$
\hat{\Phi}_{j}^{\dagger}(Y \mid X)=\hat{\bar{\Phi}}_{j}(Y \mid X),
$$

where $\nmid$ denotes Hermitian conjugation, it follows that

$$
\hat{\mathcal{T}}_{j k}^{\dagger}\left(Y_{1}, Y_{2} \mid X\right)=\hat{\mathcal{T}}_{k j}\left(Y_{2}, Y_{1} \mid X\right)
$$

and, hence,

$$
\hat{\mathcal{T}}_{j k}^{\dagger}\left(Y_{1}, Y_{2} \mid X\right)=i^{\mathfrak{p}_{j, k}+2 s} \hat{\mathcal{T}}_{j k}\left(Y_{1}, Y_{2} \mid X\right),
$$

since spin $s$ of a current is defined as the homogeneity degree in $Y$

$$
\hat{\mathcal{T}}_{j k}\left(\lambda Y_{1}, \lambda Y_{2} \mid X\right)=\lambda^{2 s} \hat{\mathcal{T}}_{j k}\left(Y_{1}, Y_{2} \mid X\right) .
$$

In particular, this implies that $\hat{\mathcal{T}}_{j k}$ is (anti)Hermitian for (odd)even spins.

Consider the full current

$$
\mathcal{J}_{\eta}\left(Y_{1}, Y_{2} \mid X\right)=\eta^{j k}(\mathcal{A}) \hat{\mathcal{T}}_{j k}\left(Y_{1}, Y_{2} \mid X\right),
$$

called later on $F$-current, with parameters $\eta^{j k}(\mathcal{A})$ depending on $\mathcal{A}_{ \pm}(Y \mid X)(2.29)$. Since $\hat{\Phi}_{j}$, $\hat{\bar{\Phi}}_{j}$ obey the unfolded equations (2.16), $\mathcal{J}_{\eta}\left(Y_{1}, Y_{2} \mid X\right)$ obeys Eq. (2.27). Hence, $\Omega\left(\mathcal{J}_{\eta}\right)$ (2.23) is a closed $M$-form, defining a conserved charge (2.25). F-currents are analogues of classical currents in unfolded dynamics. Space-time currents are

$$
\mathcal{J}_{\eta}(X)=\left.\mathcal{J}_{\eta}\left(Y_{1}, Y_{2} \mid X\right)\right|_{Y=0} .
$$

To relate statistics of $\eta^{j k}$ to that of $\hat{\mathcal{T}}_{j k}$, i.e., $\mathfrak{p}_{j, k}$ (4.13), we require

$$
\mathcal{J}_{\eta}\left(-Y_{1},-Y_{2} \mid X\right)=\mathcal{J}_{\eta}\left(Y_{1}, Y_{2} \mid X\right): \quad \eta^{j k}(-\mathcal{A})=(-1)^{\mathfrak{p}_{j, k}} \eta^{j k}(\mathcal{A})
$$


with Grassmann (odd)even $\eta^{j k}$ for $\mathfrak{p}_{j, k}=(1) 0$, i.e.,

$$
\eta^{j k}(\mathcal{A}(Y \mid X)) \eta^{\prime m n}\left(\mathcal{A}\left(Y^{\prime} \mid X^{\prime}\right)\right)=(-1)^{\mathfrak{p}_{j, k} \mathfrak{p}_{m, n}} \eta^{\prime m n}\left(\mathcal{A}\left(Y^{\prime} \mid X^{\prime}\right)\right) \eta^{j k}(\mathcal{A}(Y \mid X))
$$

In addition, symmetry parameters are required to have the same $Y$-parities as elementary fields $\eta^{k m}\left(\mathcal{A}_{+}\left(Y_{1} \mid X\right), \mathcal{A}_{-}\left(Y_{2} \mid X\right)\right)=(-1)^{\mathfrak{p}_{k}} \eta^{k m}\left(\mathcal{A}_{+}\left(-Y_{1} \mid X\right), \mathcal{A}_{-}\left(Y_{2} \mid X\right)\right)=(-1)^{\mathfrak{p}_{m}} \eta^{k m}\left(\mathcal{A}_{+}\left(Y_{1} \mid X\right), \mathcal{A}_{-}\left(-Y_{2} \mid X\right)\right)$.

Taking into account properties $(2.30)$ of $\mathcal{A}$, let $\rho$ act on parameters $\eta^{j k}(\mathcal{A})$ as

$$
\rho\left(\eta^{j k}\left(\mathcal{A}_{+}\left(Y_{1} \mid X\right), \mathcal{A}_{-}\left(Y_{2} \mid X\right)\right)\right)=i^{\mathfrak{p}_{j, k}} \eta^{k j}\left(i \mathcal{A}_{-}\left(Y_{2} \mid X\right), i \mathcal{A}_{+}\left(Y_{1} \mid X\right)\right)
$$

Hence, by virtue of (4.21), (4.23) and (4.24), the action of $\rho$ remains involutive on $\eta$. As a result,

$$
\eta=\eta^{+}+\eta^{-}, \quad \eta^{ \pm}=\frac{1}{2}(\eta \pm \rho(\eta))
$$

The terms with $\eta^{-}$do not contribute to the space-time currents $\mathcal{J}_{\eta(X)}$. Indeed, due to (2.30)

$$
\begin{aligned}
\left.\mathcal{J}_{\eta^{ \pm}}(Y \mid X)\right|_{Y=0} & =\left.\eta^{ \pm j k}\left(\mathcal{A}_{+}\left(Y_{1} \mid X\right), \mathcal{A}_{-}\left(Y_{2} \mid X\right)\right) \hat{\mathcal{T}}_{j k}\left(Y_{1}, Y_{2} \mid X\right)\right|_{Y=0} \\
& = \pm\left.\eta^{ \pm j k}\left(\mathcal{A}_{+}\left(i Y_{2} \mid X\right), \mathcal{A}_{-}\left(i Y_{1} \mid X\right)\right) \hat{\mathcal{T}}_{j k}\left(i Y_{2}, i Y_{1} \mid X\right)\right|_{Y=0}= \pm\left.\mathcal{J}_{\eta^{ \pm}}(Y \mid X)\right|_{Y=0}
\end{aligned}
$$

since the substitution $Y \Rightarrow i Y$ along with the exchange $1 \Leftrightarrow 2$ has no effect at $Y_{1,2}=0$.

More generally, we introduce $A$-currents that contain different frequency parts and can be used for computation of amplitudes

$$
\mathrm{J}_{\gamma}\left(Y_{1}, Y_{2} \mid X\right)=\sum_{a, b} \mathrm{~J}_{\gamma}^{a b}\left(Y_{1}, Y_{2} \mid X\right)
$$

where

$$
\mathrm{J}_{\gamma}^{a b}\left(Y_{1}, Y_{2} \mid X\right)=\left(\kappa_{1}^{a}\right)^{\mathfrak{p}_{j}}\left(\kappa_{2}^{b}\right)^{\mathfrak{p}_{k}} \gamma_{a b}^{j k}\left(\mathcal{A}_{+}\left(Y_{1} \mid X\right), \mathcal{A}_{-}\left(Y_{2} \mid X\right)\right) \hat{\mathrm{T}}_{j k}^{a b}\left(\kappa_{1}^{a} Y_{1}, \kappa_{2}^{b} Y_{2} \mid X\right)
$$

with arbitrary $\gamma_{a b}^{j k}\left(\mathcal{A}_{+}\left(Y_{1} \mid X\right), \mathcal{A}_{-}\left(Y_{2} \mid X\right)\right)$.

$F$-current $\mathcal{J}_{\eta}$ is a particular case of $\mathrm{J}_{\gamma}$ at $\gamma_{a b}^{k j}=\eta^{k j} \forall a, b$, i.e.,

$$
\mathcal{J}_{\eta}=\sum_{a, b} \mathcal{J}_{\eta}^{a b}, \quad \mathcal{J}_{\eta}^{a b}\left(Y_{1}, Y_{2} \mid X\right)=\left.\mathrm{J}_{\gamma}^{a b}\left(Y_{1}, Y_{2} \mid X\right)\right|_{\gamma_{a b}=\eta}
$$

The action of $\rho$ on parameters $\gamma_{a b}^{j k}\left(\mathcal{A}_{+}\left(Y_{1} \mid X\right), \mathcal{A}_{-}\left(Y_{2} \mid X\right)\right)$ is defined analogously

$$
\rho\left(\gamma_{a b}^{j k}\left(\mathcal{A}_{+}\left(Y_{1} \mid X\right), \mathcal{A}_{-}\left(Y_{2} \mid X\right)\right)\right)=i^{\mathfrak{p}_{j, k}} \gamma_{b a}^{k j}\left(i \mathcal{A}_{-}\left(Y_{2} \mid X\right), i \mathcal{A}_{+}\left(Y_{1} \mid X\right)\right)
$$

Again,

$$
\gamma=\gamma^{+}+\gamma^{-}, \quad \gamma^{ \pm}=\frac{1}{2}(\gamma \pm \rho(\gamma))
$$


Analogously to (4.22), we require

$$
\gamma_{a b}^{j k}(\mathcal{A}(Y \mid X)) \gamma_{c d}^{\prime m n}\left(\mathcal{A}\left(Y^{\prime} \mid X\right)\right)=(-1)^{\mathfrak{p}_{j, k} \mathfrak{p}_{m, n}} \gamma_{c d}^{\prime m n}\left(\mathcal{A}\left(Y^{\prime} \mid X\right)\right) \gamma_{a b}^{j k}(\mathcal{A}(Y \mid X)) .
$$

Now we are in a position to consider algebra of quantum charges and OPE of currents. The current operator algebra contains all quantum $2 r$-linear currents (2.49) which are normalordered products of the bilinear ones

$$
\mathcal{J}_{\eta_{1} \ldots \eta_{r}}^{2 r}\left(Y_{1}, \ldots, Y_{2 r} \mid X_{1}, \ldots, X_{r}\right)=\eta^{j_{1} k_{1} ; \ldots ; j_{r} k_{r}}\left(\mathcal{A}_{1}, \ldots, \mathcal{A}_{r}\right) \hat{\mathcal{T}}_{j_{1} k_{1} ; \ldots ; j_{r} k_{r}}\left(Y_{1}, \ldots, Y_{2 r} \mid X_{1}, \ldots, X_{r}\right),
$$

where

$$
\begin{array}{r}
\eta^{j_{1} k_{1} ; \ldots ; j_{r} k_{r}}\left(\mathcal{A}_{1}, \ldots, \mathcal{A}_{r}\right)=\prod_{\alpha=1}^{r}\left(\eta_{\alpha}^{j_{\alpha} k_{\alpha}}\left(\mathcal{A}_{+}\left(Y_{2 \alpha-1} \mid X_{\alpha}\right), \mathcal{A}_{-}\left(Y_{2 \alpha} \mid X_{\alpha}\right)\right)\right), \\
\hat{\mathcal{T}}_{j_{1} k_{1} ; \ldots ; j_{r} k_{r}}\left(Y_{1}, \ldots, Y_{2 r} \mid X_{1}, \ldots, X_{r}\right)=:\left(\prod_{\alpha=1}^{r} \hat{\mathcal{T}}_{j_{\alpha} k_{\alpha}}\left(Y_{2 \alpha-1}, Y_{2 \alpha} \mid X_{\alpha}\right)\right):
\end{array}
$$

\section{Algebra of charges}

\section{$5.1 \quad$ Charges}

As shown in Section थ, nonzero charges $Q$ and $\widetilde{Q}(2.25)$ are supported by currents with parameters $\eta(\mathcal{B})$ and $\widetilde{\eta}(\widetilde{\mathcal{B}})$ with $\mathcal{B}(2.34)$ and $\widetilde{\mathcal{B}}(2.35)$, respectively. The decomposition (4.27) of currents induces a similar decomposition of the differential forms (2.23) and charges (2.25)

$$
Q_{\eta}=\sum_{a b} Q_{\eta}^{a b}, \quad Q_{\eta}^{a b}=\frac{1}{2} \int_{\Sigma^{a b}} \Omega\left(J_{\eta}^{a b}\right)
$$

and similarly for $\widetilde{Q}$.

Let us first consider the charges $Q$ with parameters $\eta(\mathcal{B})$ independent of $\widetilde{\mathcal{B}}$. In this case,

$\Omega\left(J_{\eta}^{a b}\right)=\left.\frac{1}{2 M !} d W^{A_{1}} \wedge \ldots \wedge d W^{A_{M}} \varepsilon_{A_{1} \ldots A_{M}} J_{\eta}^{a b}(U, V \mid X)\right|_{U=0}, \quad d W^{A}=i d X^{A B} \frac{\partial}{\partial U^{B}}+d V^{A}$,

where $\varepsilon_{A_{1} \ldots A_{M}}$ is the fully antisymmetric tensor $\left(\varepsilon_{1 \ldots M}=1\right)$ and

$$
J_{\eta}^{a b}(U, V \mid X)=\mathcal{J}_{\eta}^{a b}(V-U, V+U \mid X)
$$

with $\mathcal{J}_{\eta}^{a b}(4.29)$. As a first step, we show that

$$
Q_{\eta}^{++}=Q_{\eta}^{--}=0, \quad Q_{\eta}=Q_{\eta}^{+-}+Q_{\eta}^{-+}=Q_{\eta^{a}}, \quad Q_{\eta^{a}}^{-+}=Q_{\eta^{a}}^{+-}, \quad a=\operatorname{sign}\left(i^{M}\right)
$$

with $\eta^{a}$ (4.25) ( $a=+,-, M$ is even).

In particular, for $M=2$, a bosonic parameter $\eta^{\operatorname{sign}\left(i^{2}\right)}$ is antisymmetric, while for $M=4$ $\eta^{\mathrm{sign}\left(i^{4}\right)}$ is symmetric. Note that, in the case of $M=4$, the fields and currents depend on 
coordinates $X^{A B}$ of the ten-dimensional matrix space. As shown in [28], global symmetry parameters of the current in the matrix space should be singular to reproduce a nonzero current in Minkowski space. This singularity is $\rho$-odd. Hence genuine symmetry parameters of the currents in Minkowski space are also $\rho$-odd. This phenomenon is expected to occur for higher $M$ as well.

Let us show that $\Omega\left(J_{\eta}^{++}\right)$(5.2) with $\eta=\eta\left(\mathcal{B}_{A}, \mathcal{B}^{A}\right)$ is exact. Indeed, by virtue of (4.2) and (4.11), for any parameter $\eta^{j k}=\eta^{j k}(\mathcal{A})$ with $\mathcal{A}(2.29) \mathcal{J}_{\eta}^{a b}$ (4.29) acquires the form

$$
\begin{gathered}
\mathcal{J}_{\eta}^{a b}\left(Y_{1}, Y_{2} \mid X\right)=\frac{\left(\kappa_{1}^{a}\right)^{\mathfrak{p}_{j}}\left(\kappa_{2}^{b}\right)^{\mathfrak{p}_{k}}}{(2 \pi)^{M}} \int_{\mathbb{R}^{2 M}} d^{M} \xi_{1} d^{M} \xi_{2}: \hat{c}_{j}^{a}\left(\xi_{1}\right) \hat{c}_{k}^{b}\left(\xi_{2}\right): \\
\eta^{j k} \exp \left(a i\left(\xi_{1 A} \xi_{1 B} X^{A B}+\kappa_{1}^{a} Y_{1}^{B} \xi_{1 B}\right)+b i\left(\xi_{2 A} \xi_{2 B} X^{A B}+\kappa_{2}^{b} Y_{2}^{B} \xi_{2 B}\right)\right),
\end{gathered}
$$

where

$\eta^{j k}\left(\mathcal{A}_{+}{ }^{A}\left(Y_{1} \mid X\right), \mathcal{A}_{+A}\left(Y_{1} \mid X\right), \mathcal{A}_{-}{ }^{A}\left(Y_{2} \mid X\right), \mathcal{A}_{-A}\left(Y_{2} \mid X\right)\right) \rightarrow \eta^{j k}\left(i \kappa_{1}^{a} \frac{\partial}{\partial \xi_{1 A}}, a i \kappa_{1}^{a} \xi_{1 A}, i \kappa_{2}^{b} \frac{\partial}{\partial \xi_{2 A}}, b i \kappa_{2}^{b} \xi_{2 A}\right)$.

Hence, by virtue of (2.34) and partial integration, (5.2) with $\eta=\left(\mathcal{B}_{A}, \mathcal{B}^{A}\right)$ gives

$$
\begin{gathered}
\Omega\left(J_{\eta}^{++}\right)=(i)^{\mathfrak{p}_{k}} \frac{1}{2(2 \pi)^{M} M !} \int_{\mathbb{R}^{2 M}} d^{M} \xi d^{M} \lambda d W^{A_{1}} \wedge \ldots \wedge d W^{A_{M}} \varepsilon_{A_{1} \ldots A_{M}} \\
\exp \left(i X^{A B}\left(\xi_{A} \xi_{B}+\lambda_{A} \lambda_{B}\right)+i V^{C}\left(i \lambda_{C}+\xi_{C}\right)\right) \eta^{j k}\left(-i(\xi-i \lambda), i \frac{\partial}{\partial(\xi+i \lambda)}\right) \hat{\mathfrak{c}}_{j}^{+}(\xi) \hat{\mathfrak{c}}_{k}^{+}(\lambda),
\end{gathered}
$$

where $d W^{A}=\left(i d X^{A B}(\xi-i \lambda)_{B}+d V^{A}\right)$. It is easy to see that $\Omega\left(J_{\eta}^{++}\right)=d \omega_{M-1}^{++}$where

$$
\begin{array}{r}
\omega_{M-1}^{++}=\frac{(i)^{\mathfrak{p}_{k}+1}}{2 M !(2 \pi)^{M}} \int_{\mathbb{R}^{2 M}} d^{M} \xi d^{M} \lambda d W^{A_{1}} \wedge \ldots \wedge d W^{A_{M-1}} \varepsilon_{A_{1} \ldots A_{M}} \frac{\mathcal{P}^{A_{M} B}\left(\xi_{B}-i \lambda_{B}\right)}{\mathcal{P}^{C D}\left(\xi_{C} \xi_{D}+\lambda_{C} \lambda_{D}\right)} \\
\exp \left(i\left(X^{A B}\left(\xi_{B}-i \lambda_{B}\right)+V^{A}\right)\left(\xi_{A}+i \lambda_{A}\right)\right) \eta^{j k}\left(-i(\xi-i \lambda), i \frac{\partial}{\partial(\xi+i \lambda)}\right) \hat{\mathfrak{c}}_{j}^{+}(\xi) \hat{\mathfrak{c}}_{k}^{+}(\lambda)
\end{array}
$$

with any positive definite matrix $\mathcal{P}^{C D}$. Here it is important that the singularity in (5.8) is integrable for $M \geq 2$. Analogously, $\Omega\left(J_{\eta}^{--}\right)$(5.2) is also exact. Hence,

$$
Q_{\eta}^{++}=Q_{\eta}^{--}=0 \text {. }
$$

To prove the remaining relations in Eq. (5.4), consider $Q_{\eta}^{+-}$. Following [35] (see also [28]), we choose the integration surface $\Sigma^{+-}$to be a $M$-dimensional plane in $\mathcal{M}_{M} \times \mathbb{C}^{M}$ parametrized by coordinates $x^{i}$ with $i=1 \ldots M$, i.e.,

$$
X^{A B}=U_{i}^{A B} x^{i}, \quad V^{A}=U^{A}=0
$$

for some $U_{i}^{A B}$. Using (5.5) and (5.10) along with (2.28) and (2.34), we obtain from (5.2)

$$
\begin{aligned}
Q_{\eta}^{+-}= & \frac{1}{2}(2 \pi)^{-M} \int_{\mathbb{R}^{M}} d^{M} x \int_{\mathbb{R}^{2 M}} d^{M}(\xi+\lambda) d^{M}(\xi-\lambda) \operatorname{det}|\mathfrak{O}\{\xi+\lambda\}| c_{j}^{+}(\xi) c_{k}^{-}(\lambda) \\
& \eta^{j k}\left(-i(\xi+\lambda),-i\left(\frac{\partial}{\partial \xi}-\frac{\partial}{\partial \lambda}\right)\right) \exp \left(i \mathfrak{O}_{n}^{A}\{\xi+\lambda\}\left(\xi_{A}-\lambda_{A}\right) x^{n}\right),
\end{aligned}
$$


where

$$
\mathfrak{O}_{n}^{A}\{\xi+\lambda\}=U_{n}^{A B}\left(\xi_{B}+\lambda_{B}\right) .
$$

Let $U_{n}^{A B}$ in (5.10) be chosen in such a way that the roots of det $|\mathfrak{O}(\xi)|$ are independent functions of $\xi$ (that is generically true). Observing that the measure in (5.11) is equivalent to $\delta^{M}(\xi-\lambda)$, we obtain

$$
Q_{\eta}^{+-}=\left.\frac{1}{2} \int d^{M} \xi \eta^{j k}\left(-2 i \xi, \frac{i}{2} \frac{\partial}{\partial \nu}\right) \hat{\mathfrak{c}}_{j}^{+}(\xi+\nu) \hat{\mathfrak{c}}_{k}^{-}(\xi-\nu)\right|_{\nu=0} .
$$

Analogously

$$
Q_{\eta}^{-+}=\left.\frac{1}{2} i^{\mathfrak{p}(\eta)+M} \int d^{M} \xi \eta^{k j}\left(-2 \xi, \frac{1}{2} \frac{\partial}{\partial \nu}\right) \hat{\mathfrak{c}}_{j}^{+}(\xi+\nu) \hat{\mathfrak{c}}_{k}^{-}(\xi-\nu)\right|_{\nu=0} .
$$

Here the factor of $i^{M}$ results from $U$-derivatives in the operator $\left(d X^{A B} \frac{\partial}{\partial U^{B}}\right)^{M}$ in (5.2).

To complete the proof of (5.4), by virtue of (2.34) and (4.24) we obtain

$$
Q_{\eta}=\left.\frac{1}{2} \int d^{M} \xi\left(1+i^{M} \rho\right) \eta^{j k}\left(-2 i \xi, \frac{i}{2} \frac{\partial}{\partial \nu}\right) \hat{\mathfrak{c}}_{j}^{+}(\xi+\nu) \hat{\mathfrak{c}}_{k}^{-}(\xi-\nu)\right|_{\nu=0} .
$$

Integration of charges over the twistor space with coordinates $V$ using

$$
\left.\eta^{m n}\left(\mathcal{B}_{A}(U, V \mid X), \mathcal{B}^{A}(U, V \mid X)\right)\right|_{X=0}=\eta^{m n}\left(\partial_{U}, V\right)
$$

gives equivalent results. However, there is a subtlety that, to keep integrals well-defined, one has to integrate different components $Q^{a b}$ over different cycles in the complex twistor space, which is possible because every $Q^{a b}$ represents a conserved charge. To give precise meaning to integrals it is useful to use the formulation in Fock-Siegel space which makes the $\xi$ integration well-defined. Skipping details, the proper definition of the charges (5.1) is

$$
\begin{aligned}
& Q_{\eta}^{+-}=\left.\frac{1}{2} \int_{\Sigma^{+-}} d^{M} v \quad \eta^{j k}\left(\partial_{u}, v\right) \hat{\mathcal{T}}_{j k}^{+-}(v-u, v+u \mid 0)\right|_{u=0}, \\
& Q_{\eta}^{++}=\left.\frac{1}{2} i^{\mathfrak{p}} \int_{\Sigma^{++}} d^{M} z \quad \eta^{j k}\left(\partial_{\bar{z}}, z\right) \hat{\mathcal{T}}_{j k}^{++}(z-\bar{z}, i(z+\bar{z}) \mid 0)\right|_{\bar{z}=0}, \\
& Q_{\eta}^{--}=\left.\frac{1}{2} i^{\mathfrak{p}} \int_{\Sigma^{--}} d^{M} z \quad \eta^{j k}\left(\partial_{\bar{z}}, z\right) \hat{\mathcal{T}}_{j k}^{--}(i(z-\bar{z}), z+\bar{z} \mid 0)\right|_{\bar{z}=0}, \\
& Q_{\eta}^{-+}=\left.\frac{1}{2} i^{M} \int_{\Sigma^{-+}} d^{M} v \quad \eta^{j k}\left(i \partial_{u}, i v\right) \hat{\mathcal{T}}_{j k}^{-+}(-v-u, u-v \mid 0)\right|_{u=0},
\end{aligned}
$$

where $\Sigma^{-+}=\Sigma^{+-}=\mathbb{R}^{M}$ while $\Sigma^{--}=\Sigma^{++}=\gamma^{M}$ where $\gamma^{M}$ is any $M$-dimensional closed cycle in $\mathbb{C}^{M}(z, \bar{z})$. With this definition, the charges $Q_{\eta}^{++}=Q_{\eta}^{--}=0$ as being represented by integrals of analytic functions. (Using formulation in Fock-Siegel space, the corresponding contours can 
be deformed to infinity where the integrals are zero.) For $Q_{\eta}^{+-}$and $Q_{\eta}^{-+}$this prescription again gives (5.4).

By (2.45), the analysis of charges $\widetilde{Q}$ is analogous, giving

$$
\begin{aligned}
\widetilde{Q}_{\widetilde{\eta}(\widetilde{\mathcal{B}})}^{+-} & =\left.\frac{1}{2} \int d^{M} \xi \widetilde{\eta}^{j k}\left(2 i \xi,-\frac{i}{2} \frac{\partial}{\partial \nu}\right) \hat{\mathfrak{c}}_{j}^{+}(\xi+\nu) \hat{\mathfrak{c}}_{k}^{-}(\xi-\nu)\right|_{\nu=0}, \\
\widetilde{Q}_{\widetilde{\eta}(\widetilde{\mathcal{B}})}^{-+} & =\left.\frac{1}{2} i^{M+\mathfrak{p}(\eta)} \int d^{M} \xi \widetilde{\eta}^{j k}\left(-2 \xi, \frac{1}{2} \frac{\partial}{\partial \nu}\right) \hat{\mathfrak{c}}_{j}^{+}(\xi+\nu) \hat{\mathfrak{c}}_{k}^{-}(\xi-\nu)\right|_{\nu=0} .
\end{aligned}
$$

By virtue of (4.24) and (2.35), the counterpart of (5.15) is

$$
\widetilde{Q}_{\widetilde{\eta}(\widetilde{\mathcal{B}})}=\left.\frac{1}{2} \int d^{M} \xi\left(1+i^{M} \rho\right) \widetilde{\eta}^{j k}\left(2 i \xi,-\frac{i}{2} \frac{\partial}{\partial \nu}\right) \hat{\mathfrak{c}}_{j}^{+}(\xi+\nu) \hat{\mathfrak{c}}_{k}^{-}(\xi-\nu)\right|_{\nu=0} .
$$

\subsection{Higher-spin algebra}

Let us show that, for $\rho$-odd $\eta$ and $\eta^{\prime}$,

$$
\left[Q_{\eta}, Q_{\eta^{\prime}}\right]=Q_{\left[\eta, \eta^{\prime}\right]_{*}},
$$

where

$$
\left[Q_{\eta}, Q_{\eta^{\prime}}\right]=Q_{\eta} Q_{\eta^{\prime}}-Q_{\eta^{\prime}} Q_{\eta}, \quad[a, b]_{*}=a * b-b * a
$$

and

$$
(a * b)^{k n}(u, v)=\delta_{j m} a^{k j}(u, v) \exp \left(\frac{\overleftarrow{\partial}}{\partial u_{C}} \frac{\vec{\partial}}{\partial v^{C}}-\frac{\overleftarrow{\partial}}{\partial v^{C}} \frac{\vec{\partial}}{\partial u_{C}}\right) b^{m n}(u, v)
$$

An important property of the star product is that it possesses the antiautomorphism $\rho$ [36]

$$
\rho\left(a^{m n}(u, v)\right):=(i)^{\mathfrak{p}(a)} a^{n m}(i u, i v), \quad a^{m n}(-u,-v)=(-1)^{\mathfrak{p}(a)} a^{m n}(u, v),
$$

i.e., assuming that the coefficients of $a(u, v)$ are endowed with the Grassmann parity $\mathfrak{p}(a)$,

$$
\rho(a * b)=\rho(b) * \rho(a) .
$$

Hence, for $\rho$-odd $a$ and $a^{\prime},\left[a, a^{\prime}\right]_{*}$ is also $\rho$-odd.

The proof of (5.24) is straightforward. By virtue of (5.4),

$$
\begin{aligned}
{\left[Q_{\eta}, Q_{\eta^{\prime}}\right]=} & \int d^{M} v d^{M} v^{\prime} \eta^{j k}\left(\partial_{u}, v\right) \eta^{\prime m n}\left(\partial_{u^{\prime}}, v^{\prime}\right) \\
& {\left.\left[\widehat{C}_{j}^{+}(v-u \mid 0) \widehat{C}_{k}^{-}(v+u \mid 0), \widehat{C}_{m}^{+}\left(v^{\prime}-u^{\prime} \mid 0\right) \widehat{C}_{n}^{-}\left(v^{\prime}+u^{\prime} \mid 0\right)\right]\right|_{u=u^{\prime}=0} }
\end{aligned}
$$

Using (4.4) along with (4.22), we obtain

$$
\begin{aligned}
{\left[Q_{\eta}, Q_{\eta^{\prime}}\right]=} & \delta_{j m} \int d^{M} v\left(\eta^{k j}\left(\partial_{u}, v-u^{\prime}\right) \eta^{\prime m n}\left(\partial_{u^{\prime}}, v+u\right)-\eta^{\prime k j}\left(\partial_{u^{\prime}}, v-u\right) \eta^{m n}\left(\partial_{u}, v+u^{\prime}\right)\right) \\
& \left.\widehat{C}_{k}^{+}\left(v^{\prime}-\left(u+u^{\prime}\right) \mid 0\right) \widehat{C}_{n}^{-}\left(v^{\prime}+u+u^{\prime} \mid 0\right)\right|_{u=u^{\prime}=0}
\end{aligned}
$$


Hence

$\left[Q_{\eta}, Q_{\eta^{\prime}}\right]=\left.\left[\int d^{M} v\left(\left(\eta * \eta^{\prime}\right)^{m j}\left(\partial_{u}, v\right)-\left(\eta^{\prime} * \eta\right)^{m j}\left(\partial_{u}, v\right)\right) \widehat{C}_{m}^{+}(v-u \mid 0) \widehat{C}_{j}^{-}(v+u \mid 0)\right]\right|_{u=0}$,

which implies (5.24) by virtue of (5.4), (5.17), (5.20).

Using that $Q$ is related to $\widetilde{Q}$ via (2.45) it can be shown that

$$
\left[\widetilde{Q}_{\tilde{\eta}}, \widetilde{Q}_{\tilde{\eta}^{\prime}}\right]=Q_{\left[K \tilde{\eta}, K \tilde{\eta}^{\prime}\right]_{*}}, \quad\left[\widetilde{Q}_{\tilde{\eta}}, Q_{\eta^{\prime}}\right]=\widetilde{Q}_{K\left[K \tilde{\eta}, \eta^{\prime}\right]_{*}}
$$

with the convention

$$
K^{2}=1, \quad K \eta=(-1)^{\mathfrak{p}(\eta)} \eta K .
$$

These commutation relations coincide with those of the HS subalgebra of the algebra of [37] described by the symmetry parameters $\eta(u, v ; K)$ that, in addition to spinor variables, depend on the Klein operator $K$ possessing properties (5.31). In other words, combining $Q$ and $\widetilde{Q}$ into $\mathcal{Q}$ with the parameter

$$
\varepsilon=\eta+K \tilde{\eta},
$$

the commutation relations (5.24) and (5.30) combine to

$$
\left[\mathcal{Q}_{\varepsilon}, \mathcal{Q}_{\varepsilon^{\prime}}\right]=\mathcal{Q}_{\left[\varepsilon, \varepsilon^{\prime}\right]_{*}}
$$

More precisely, in the case of $M=2$, the resulting algebra of charges $\mathcal{Q}$ is isomorphic to the HS algebra $h o(\mathcal{N}, \mathcal{N} \mid 4)$ in notations of [38. In this paper, it appears as the $3 d$ conformal HS algebra acting on $3 d$ conformal fields realized as rank-one $M=2$ fields of Section 2 . In accordance with consideration of [38], the doubling of fields is because the $3 d$ conformal HS symmetry acts both on bosons and fermions. In particular, its even subalgebra decomposes into direct sum of two isomorphic algebras acting on $3 d$ massless scalar and spinor, respectively. All other types of HS algebras $h u(n, m \mid 4), h o(n, m \mid 4)$ and $h u s p(n, m \mid 4)$ can be obtained along the lines of [38] as proper subalgebras of $h o(\mathcal{N}, \mathcal{N} \mid 4)$ preserving appropriate additional structures (bilinear forms or gradings) with respect to color indices.

Note that multiparticle algebra $\mathbf{M}$ of [16] can be realized as the algebra of all polynomials of $Q_{\varepsilon}$ with various $\varepsilon$, that includes unity. By virtue of (5.33) it can be realized as the algebra of symmetrized polynomials of $\varepsilon$, or, equivalently, as the algebra of polynomials of a single $\varepsilon$. This is the realization considered in [16].

\section{Twistor current operator algebra}

Free bilinear twistor currents are most conveniently described as

$$
\mathcal{I}_{g}^{2}=\sum_{a, b=+,-} \int d^{2 M} W g_{a b}^{m n}\left(W_{1}, W_{2}\right) \hat{\mathrm{T}}_{m n}^{a b}\left(W_{1}, W_{2} \mid 0\right)
$$


with $\hat{\mathrm{T}}_{k j}^{a b}\left(W_{1}, W_{2} \mid X\right)$ (4.8). Symmetry properties (4.10) of $\hat{\mathrm{T}}_{m n}^{a b}\left(W_{1}, W_{2} \mid 0\right)$ imply that

$$
\begin{aligned}
g_{a b}^{m n}\left(W_{1}, W_{2}\right) & =(-1)^{\mathfrak{p}_{m}} g_{a b}^{m n}\left(-W_{1}, W_{2}\right)=(-1)^{\mathfrak{p}_{n}} g_{a b}^{m n}\left(W_{1},-W_{2}\right), \\
\mu(g) & =g
\end{aligned}
$$

where

$$
\mu(g)_{a b}^{m n}\left(W_{1}, W_{2}\right):=(-1)^{\mathfrak{p}_{m} \mathfrak{p}_{n}} g_{b a}^{n m}\left(W_{2}, W_{1}\right)
$$

Also, we require

$$
g_{b a}^{n m} g_{c d}^{\prime j k}=(-1)^{\mathfrak{p}_{n, m} \mathfrak{p}_{j, k}} g_{c d}^{\prime j k} g_{b a}^{n m}
$$

with $\mathfrak{p}_{j, k}$ (4.13). Otherwise, the function $g\left(W_{1}, W_{2}\right)$ remains arbitrary. Note that due to (6.4)

$$
: \mathcal{I}_{f}^{2} \mathcal{I}_{g}^{2}:=: \mathcal{I}_{g}^{2} \mathcal{I}_{f}^{2}: \quad \forall g, f .
$$

In Section 8, using evolution formula (3.14), the space-time dependent current will be represented as the twistor current with the function $g\left(W_{1}, W_{2}\right)$ that depends in a specific way on space-time coordinates as parameters. This trick fully reduces construction of space-time operator product algebra to the much simpler problem in the twistor space. More generally, via uplifting of twistor-to-boundary $\mathcal{D}$-functions to twistor-to-bulk ones as discussed in the end of Section 3, it can be straightforwardly extended to the bulk where the boundary conformal algebra acts.

\subsection{OPE of twistor currents}

A basis of current operator algebra consists of $2 n$ twistor currents with various $n$

$$
: \prod_{j=1}^{n} \mathcal{I}_{g_{j}}^{2}:, \quad \mathcal{I}^{0}=I d .
$$

Since currents (6.6) are symmetric multilinear functionals of $g_{j}$, the twistor operator algebra can be represented by nonlinear functionals of a single function $g\left(W_{1}, W_{2}\right)$ (skipping color indices). This leads to the construction of algebra $\mathbf{M}$ described in [16]. To determine full current operator algebra it suffices to compute $\mathcal{I}^{2 n} \mathcal{I}^{2}$ for any $n$. Though the final results can be derived by methods of [16], here we will use a slightly different setup based on interesting algebraic structures most appropriate for description of $n$-point functions.

The cases of $A$-currents with the parameters $g_{a b}^{m n}\left(W_{1}, W_{2}\right)$, that are independent for different values of indices $a, b$ modulo symmetry properties (6.2), and $F$-currents with $g_{a b}^{m n}\left(W_{1}, W_{2}\right)$ at different $a, b$ related to each other in accordance with (4.5), are different. We start with the more general case of $A$-currents.

From the commutation relations (4.4) along with (6.2) and (6.4) it follows that

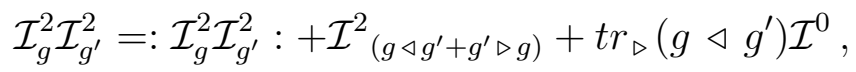

where

$$
\left(g \triangleleft g^{\prime}\right)_{a b}^{m n}\left(W_{1}, W_{2}\right)=2 \delta_{k j} \tau^{d c} \int d p g_{a c}^{m k}\left(W_{1}, p\right) g_{d b}^{\prime j n}\left(p, W_{2}\right),
$$




$$
\begin{gathered}
\left(g \triangleright g^{\prime}\right)_{a b}^{m n}\left(W_{1}, W_{2}\right)=2 \delta_{k j} \tau^{c d} \int d p g_{a c}^{m k}\left(W_{1},-p\right) g_{d b}^{\prime j n}\left(p, W_{2}\right), \\
\tau^{a b}=\delta_{+}^{a} \delta_{-}^{b}
\end{gathered}
$$

and

$$
t r_{\triangleleft}(g)=\delta_{m n} \tau^{a b} \int d p g_{a b}^{m n}(-p, p), \quad t r_{\triangleright}(g)=\delta_{m n} \tau^{b a} \int d p g_{a b}^{m n}(p, p) .
$$

The product laws $\triangleleft$ and $\triangleright$ are both associative similarly to the product of matrices $\left(h h^{\prime}\right)_{a b}=$ $h_{a c} t^{c d} h_{d b}^{\prime}$ with some $t^{c d}$. Moreover, they are mutually associative, i.e.,

$$
\begin{aligned}
& \left(\left(g \triangleright g^{\prime}\right) \triangleleft f\right)_{a b}^{m n}\left(W_{1}, W_{2}\right)=\left(g \triangleright\left(g^{\prime} \triangleleft f\right)\right)_{a b}^{m n}\left(W_{1}, W_{2}\right), \\
& \left(g \triangleleft\left(g^{\prime} \triangleright f\right)\right)_{a b}^{m n}\left(W_{1}, W_{2}\right)=\left(\left(g \triangleleft g^{\prime}\right) \triangleright f\right)_{a b}^{m n}\left(W_{1}, W_{2}\right),
\end{aligned}
$$

hence providing an example of a biassociative algebra discussed in [16]. One can see that

$$
\operatorname{tr}_{\triangleright}(g)=t r_{\triangleleft}(\mu(g)),
$$

and

$$
t r_{\triangleleft}(g \triangleleft f)=t r_{\triangleleft}(f \triangleleft g), \quad t r_{\triangleright}(g \triangleright f)=t r_{\triangleright}(f \triangleright g),
$$

i.e., $t r_{\triangleleft}$ and $t r_{\triangleright}$ are supertraces on the algebras $A_{\triangleleft}$ and $A_{\triangleright}$ with the respective products. In addition, the following relation holds

$$
t r_{\triangleright}(g \triangleleft f)=t r_{\triangleleft}(f \triangleright g)
$$

By virtue of (6.3) and (6.4),

$$
g \triangleright g^{\prime}=\mu\left(\mu\left(g^{\prime}\right) \triangleleft \mu(g)\right) .
$$

From (6.16) it follows in particular that if $g$ and $g^{\prime}$ obey (6.2), $\left(g \triangleleft g^{\prime}+g^{\prime} \triangleright g\right)$ also does, which, in fact, is the reason for appearance of this combination in Eq. (6.7). Also note that, by virtue of $(\underline{6.13})$ and $(6.16)$,

$$
\operatorname{tr}_{\triangleleft}(g \triangleleft f)=\operatorname{tr}_{\triangleright}(g \triangleright f), \quad \forall g=\mu(g), f=\mu(f) .
$$

Consider the commutator

$$
\left[\mathcal{I}_{g}^{2}, \mathcal{I}_{g^{\prime}}^{2}\right]=\mathcal{I}_{g}^{2} \mathcal{I}_{g^{\prime}}^{2}-\mathcal{I}_{g^{\prime}}^{2} \mathcal{I}_{g}^{2}
$$

Because of Eq. (6.7), $\mathcal{I}^{4}$ does not contribute. Taking into account (6.15) we obtain

$$
\left[\mathcal{I}_{g}^{2}, \mathcal{I}_{g^{\prime}}^{2}\right]=\mathcal{I}_{\left[g, g^{\prime}\right]_{\star}}^{2}+\frac{1}{2}\left(t r_{\triangleright}+t r_{\triangleleft}\right)\left(\left[g, g^{\prime}\right]_{\star}\right) \mathcal{I}^{0}
$$

where

$$
\begin{gathered}
{\left[g, g^{\prime}\right]_{\star}=g \star g^{\prime}-g^{\prime} \star g} \\
\left(g \star g^{\prime}\right)_{a b}^{m n}\left(W_{1}, W_{2}\right)=\left(g \triangleleft g^{\prime}\right)_{a b}^{m n}\left(W_{1}, W_{2}\right)-\left(g \triangleright g^{\prime}\right)_{a b}^{m n}\left(W_{1}, W_{2}\right) .
\end{gathered}
$$


The product law $\star$ is associative. More generally, from (6.12) it follows that all linear combinations of $\triangleleft$ and $\triangleright$ are mutually associative, forming a particular case of multi-associative algebra by which we mean a linear space $A$ over the field $\mathbb{K}$ endowed with a set of such mutually associative products $*_{1}, \ldots, *_{k}$ that any their linear combination $\alpha^{1} *_{1}+\ldots+\alpha^{k_{*}}, \alpha^{i} \in \mathbb{K}$ is associative. In particular, it is useful to introduce

$$
\left(g \cdot g^{\prime}\right)_{a b}^{m n}\left(W_{1}, W_{2}\right)=\left(g \triangleleft g^{\prime}\right)_{a b}^{m n}\left(W_{1}, W_{2}\right)+\left(g \triangleright g^{\prime}\right)_{a b}^{m n}\left(W_{1}, W_{2}\right) .
$$

Analogously to the product (6.7) of two bilinear currents, it is not difficult to obtain the product of a $2 n$-linear current (6.6) and a bilinear current using (4.4), (6.2), (6.4) and (6.12)

$$
\begin{gathered}
: \mathcal{I}_{g_{1}}^{2} \ldots \mathcal{I}_{g_{n}}^{2}: \mathcal{I}_{f}^{2}=: \mathcal{I}_{g_{1}}^{2} \ldots \mathcal{I}_{g_{n}}^{2} \mathcal{I}_{f}^{2}:+\sum_{k}: \mathcal{I}_{g_{1}}^{2} \ldots \widehat{\mathcal{I}_{g_{k}}^{2}} \ldots \mathcal{I}_{g_{n}}^{2} \mathcal{I}_{\left(g_{k} \triangleleft f+f \triangleright g_{k}\right)}^{2}: \\
+\sum_{k} \operatorname{tr}_{\triangleright}\left(g_{k} \triangleleft f\right): \mathcal{I}_{g_{1}}^{2} \ldots \widehat{\mathcal{I}_{g_{k}}^{2}} \ldots \mathcal{I}_{g_{n}}^{2}:+\sum_{k>p}: \mathcal{I}_{g_{1}}^{2} \ldots \widehat{\mathcal{I}_{g_{p}}^{2}} \ldots \widehat{\mathcal{I}_{g_{k}}^{2}} \ldots \mathcal{I}_{g_{n}}^{2} \mathcal{I}_{\left(g_{k} \triangleleft f \triangleright g_{p}+g_{p} \triangleleft f \triangleright g_{k}\right)}^{2}:,
\end{gathered}
$$

where $\widehat{\mathcal{I}_{g_{k}}^{2}}, \widehat{\mathcal{I}_{g_{p}}^{2}}$ denote omitted terms.

Using (6.12), (6.14), (6.15) and (6.21), one can obtain, in particular,

$$
\begin{aligned}
\mathcal{I}_{g_{1}}^{2} \mathcal{I}_{g_{2}}^{2} \mathcal{I}_{g_{3}}^{2} & =: \mathcal{I}_{g_{1}}^{2} \mathcal{I}_{g_{2}}^{2} \mathcal{I}_{g_{3}}^{2}:+: \mathcal{I}_{g_{1}}^{2} \mathcal{I}^{2}{ }_{g_{2} \triangleleft g_{3}}:+: \mathcal{I}_{g_{1}}^{2} \mathcal{I}^{2}{ }_{g_{3} \triangleright g_{2}}: \\
& +: \mathcal{I}_{g_{2}}^{2} \mathcal{I}^{2}{ }_{g_{1} \triangleleft g_{3}}:+: \mathcal{I}_{g_{2}}^{2} \mathcal{I}^{2}{ }_{g_{3} \triangleright g_{1}}:+: \mathcal{I}_{g_{3}}^{2} \mathcal{I}^{2}{ }_{g_{1} \triangleleft g_{2}}:+: \mathcal{I}_{g_{3}}^{2} \mathcal{I}_{g_{2} \triangleright g_{1}}^{2}: \\
& +\mathcal{I}^{2}{ }_{g_{1} \triangleleft g_{3} \triangleright g_{2}}+\mathcal{I}^{2}{ }_{g_{3} \triangleright g_{1} \triangleleft g_{2}}+\mathcal{I}^{2}{ }_{g_{2} \triangleright g_{1} \triangleleft g_{3}} \\
& +\mathcal{I}^{2}{ }_{g_{2} \triangleleft g_{3} \triangleright g_{1}}+\mathcal{I}^{2}{ }_{g_{1} \triangleleft g_{2} \triangleleft g_{3}}+\mathcal{I}^{2}{ }_{g_{3} \triangleright g_{2} \triangleright g_{1}} \\
& +\operatorname{tr}_{\triangleright}\left(g_{1} \triangleleft g_{2}\right) \mathcal{I}_{g_{3}}^{2}+\operatorname{tr}_{\triangleright}\left(g_{2} \triangleleft g_{3}\right) \mathcal{I}_{g_{1}}^{2}+\operatorname{tr}_{\triangleright}\left(g_{1} \triangleleft g_{3}\right) \mathcal{I}_{g_{2}}^{2} \\
& +\operatorname{tr}_{\triangleright}\left(g_{1} \triangleleft g_{2} \triangleleft g_{3}\right) \mathcal{I}^{0}+\operatorname{tr}_{\triangleleft}\left(g_{3} \triangleright g_{2} \triangleright g_{1}\right) \mathcal{I}^{0} .
\end{aligned}
$$

Analogously, Eq. (6.21) allows us to derive in Section 6.4 the explicit formula for the product of any number of bilinear currents $\mathcal{I}_{g_{1}}^{2} \ldots \mathcal{I}_{g_{n}}^{2}$.

\subsection{Star-product interpretation}

Current equation (2.22) is mapped to a first-order PDE system by the half-Fourier transform [28]. A similar transformation is known to map the convolution product to Weyl-Moyal star product (see, e.g., [39]).

Indeed, half-Fourier transform can be introduced as follows

$$
\begin{aligned}
\widetilde{g}(W, V) & =(2 \pi)^{-M / 2} \int d^{M} U \exp \left(i W_{C} U^{C}\right) g(V-U, V+U), \\
g(V-U, V+U) & =(2 \pi)^{-M / 2} \int d^{M} W \exp \left(-i W_{C} U^{C}\right) \widetilde{g}(W, V),
\end{aligned}
$$

or, alternatively,

$$
\begin{aligned}
\ddot{g}(U, W) & =(2 \pi)^{-M / 2} \int d^{M} V \exp \left(i W_{C} V^{C}\right) g(V-U, V+U), \\
g(V-U, V+U) & =(2 \pi)^{-M / 2} \int d^{M} W \exp \left(-i W_{C} V^{C}\right) \ddot{g}(U, W) .
\end{aligned}
$$


Note that, for functions of definite symmetry $G(U, V)=(-1)^{\pi} G(V, U)$,

$$
\widetilde{G}(W, V)=(-1)^{\pi} \ddot{G}(W, V) .
$$

Direct substitution of (6.23) into 6.8) gives

$$
\left(\widetilde{g \triangleleft g^{\prime}}\right)_{a b}^{m n}(W, V)=2^{1+3 M / 2} \pi^{M / 2} \delta_{k j}\left(\widetilde{g}_{a-}^{m, k} * \widetilde{g}_{+b}^{j, n}\right)(W, V),
$$

where

$F * G(x, y)=(2 \pi)^{-2 M} \int d s d t d u d v \exp \left[i\left(u_{C} v^{C}-t_{C} s^{C}\right)\right] F(x+t, y+v) G(x+u, y+s)$,

which is equivalent to the star product (5.25). In these terms, (6.11) reads as

$$
t r_{\triangleleft}(g)=(2 \pi)^{M / 2} \delta_{n m} \tilde{g}_{+-}^{n m}(0,0), \quad \operatorname{tr}_{\triangleright}(g)=(2 \pi)^{M / 2} \delta_{n m} \ddot{g}_{-+}^{n m}(0,0) .
$$

Thus, the half-Fourier transform relates the formalism of convolution product convenient for the analysis of boundary currents to the star-product formalism familiar for the bulk analysis of HS theories [20]. In fact, in the framework of bulk HS gauge theory, the half-Fourier transform also plays crucial role, relating adjoint and twisted adjoint representations of HS algebra. In the bulk formalism, various (half-)Fourier transforms are naturally represented by the Klein operators (see, e.g., [10]).

\subsection{Butterfly algebras}

For any associative algebra $A_{\star}$, butterfly algebra $A_{B}$ is the unital associative algebra spanned by elements $g_{j_{1}, \ldots, j_{k}} \in A_{\star}$ endowed with a set of integers $j_{1}, \ldots, j_{k}$. Given three elements $B_{+}, B_{-}, B_{0} \in A_{\star}$, the butterfly product law $\stackrel{B}{\bowtie}$ is defined as follows

$$
g_{j_{1}, \ldots, j_{k}} \bowtie f_{i_{1}, \ldots, i_{m}}= \begin{cases}g_{j_{1}, \ldots, j_{k}} \star B_{-} \star f_{i_{1}, \ldots, i_{m}} & \text { if } j_{k}<i_{1}, \\ g_{j_{1}, \ldots, j_{k}} \star B_{+} \star f_{i_{1}, \ldots, i_{m}} & \text { if } j_{k}>i_{1}, \\ g_{j_{1}, \ldots, j_{k}} \star B_{0} \star f_{i_{1}, \ldots, i_{m}} & \text { if } j_{k}=i_{1} .\end{cases}
$$

$\aleph_{\bowtie}^{B}$ is associative by virtue of associativity of $\star$. Unit element $e_{B}$ of $A_{B}$ is identified with $g_{j_{1}, \ldots, j_{k}}$ with the empty set of indices, i.e., $k=0$. Trace $t r_{\star}$ of $A_{\star}$ induces a trace of $A_{B}$

$$
\begin{aligned}
& \operatorname{tr}_{\bowtie}\left(g_{j_{1}, \ldots, j_{k}}\right)= \begin{cases}\operatorname{tr}_{\star}\left(B_{+} \star g_{j_{1}, \ldots, j_{k}}\right) & \text { if } j_{1}<j_{k}, \\
\operatorname{tr}_{\star}\left(B_{-} \star g_{j_{1}, \ldots, j_{k}}\right) & \text { if } j_{1}>j_{k}, \\
\operatorname{tr}_{\star}\left(B_{0} \star g_{j_{1}, \ldots, j_{k}}\right) & \text { if } j_{1}=j_{k} \text { or } k \leq 1,\end{cases} \\
& \operatorname{tr}_{\triangleright}\left(g_{j_{1}, \ldots, j_{k}} \bowtie f_{i_{1}, \ldots, i_{m}}\right)=\operatorname{tr}_{\bowtie}\left(f_{i_{1}, \ldots, i_{m}} \stackrel{B}{\bowtie} g_{j_{1}, \ldots, j_{k}}\right) .
\end{aligned}
$$


Clearly, products $\stackrel{B_{1}}{\bowtie}$ and $\stackrel{B_{2}}{\bowtie}$ with any two sets $B_{1}=\left(B_{1+}, B_{1-}, B_{10}\right)$ and $B_{2}=\left(B_{2+}, B_{2-}, B_{20}\right)$ of elements of $A_{\star}$ are mutually associative and

$$
\operatorname{tr}_{\bowtie}\left(g \stackrel{B_{2}}{\bowtie} f\right)=\operatorname{tr}_{\underset{B_{2}}{\bowtie}}\left(f \stackrel{B_{1}}{\bowtie} g\right)
$$

As shown below, multiple products of $J^{2 n}$ currents can be described by the butterfly algebra $A_{\Perp}$ with

$$
\unrhd=\stackrel{B}{\bowtie}, \quad B_{0}=B_{+}=-\Pi_{+}, B_{-}=\Pi_{-},
$$

where $\Pi_{ \pm}$are defined so that

$$
\Pi_{+}+\Pi_{-}=e_{\star}, \quad f \triangleright g=-f \star \Pi_{+} \star g, \quad f \triangleleft g=f \star \Pi_{-} \star g .
$$

Note that, as shown in [16], $\Pi_{ \pm}$are projectors, i.e., $\Pi_{ \pm} \star \Pi_{ \pm}=\Pi_{ \pm}$.

We will also use the following products

$$
\stackrel{\triangleleft}{\bowtie}=\stackrel{B}{\bowtie}: \quad B_{0}=B_{ \pm}=\Pi_{-}, \quad \stackrel{\triangleright}{\bowtie}=\stackrel{B}{\bowtie}: \quad B_{0}=B_{ \pm}=-\Pi_{+}, \quad \bar{\bowtie}=: \quad B_{+}=B_{0}=0, B_{-}=e_{\star},
$$

where $e_{\star}$ is the unit element of $A_{\star}$. Instead of the product $\stackrel{B}{\bowtie}$ with $B_{0}=B_{ \pm}=e_{\star}$ we will use notation $\star$. Note that, by (6.34),

$$
\stackrel{\bowtie}{\bowtie}=\star, \quad \bar{\bowtie}=\bowtie-\bowtie .
$$

For any $B, A_{\triangleright}$ contains ideals $I_{\triangleright}^{k} \subset A_{B_{\triangleright}}$ spanned by such its elements, that contain at least $k$ coinciding successive indices $j_{n}=j_{n+1}=j_{n+2}=\ldots=j_{n+k-1}$. Algebras $A_{\triangleright}^{k}=A_{\triangleright} / I_{\triangleright}^{k+1}$ are spanned by elements that do not contain more than $k$ coinciding successive indices.

It turns out that multiple current products are most compactly described by the butterfly algebra $A_{\bowtie} \sim A_{\bowtie} / I_{\bowtie}^{2}$ spanned by $g_{a b}^{n m}{ }_{j_{1}, \ldots, j_{k}}\left(W_{1}, W_{2}\right)$ endowed with ordered sets of integers $j_{1}, \ldots, j_{k}$ such that $j_{n} \neq j_{n+1} \forall n$. The butterfly product law is

$$
g_{j_{1}, \ldots, j_{k}} \bowtie g_{i_{1}, \ldots, i_{m}}=\theta\left(i_{1}-j_{k}\right) g_{j_{1}, \ldots, j_{k}} \triangleleft g_{i_{1}, \ldots, i_{m}}+\theta\left(j_{k}-i_{1}\right) g_{j_{1}, \ldots, j_{k}} \triangleright g_{i_{1}, \ldots, i_{m}},
$$

where

$$
\theta(n)=1 \quad \text { at } n>0, \quad \theta(n)=0 \quad \text { at } n \leq 0 .
$$

$A_{\bowtie}$ possesses a trace

$$
\operatorname{tr} r_{\bowtie}\left(g_{j_{1}, \ldots, j_{k}}\right)=\theta\left(j_{k}-j_{1}\right) \operatorname{tr} r_{\triangleright}\left(g_{j_{1}, \ldots, j_{k}}\right)+\theta\left(j_{1}-j_{k}\right) \operatorname{tr} r_{\triangleleft}\left(g_{j_{1}, \ldots, j_{k}}\right) .
$$

A useful property is that, for $j_{m}=\max \left(j_{1}, \ldots, j_{k}\right)$,

$$
\operatorname{tr}_{\bowtie}\left(g_{j_{1}} \ldots \bowtie g_{j_{k}}\right)=\operatorname{tr}_{\triangleright}\left(g_{j_{m+1}} \bowtie \ldots \bowtie g_{j_{k}} \bowtie g_{j_{1}} \bowtie \ldots \bowtie g_{j_{m-1}} \triangleleft g_{j_{m}}\right) .
$$




\subsection{Butterfly formulae for multiple products and $n$-point functions}

In terms of butterfly algebra $A_{\bowtie}$, operator products $(6.7)$ and (6.22) can be rewritten as

$$
\begin{aligned}
\mathcal{I}_{g_{1}}^{2} \mathcal{I}_{g_{2}}^{2} & =\sum_{i_{j}=1,2 ; i_{1} \neq i_{2}}\left\{\frac{1}{2 !}: \mathcal{I}_{g_{i_{1}}}^{2} \mathcal{I}_{g_{i_{2}}}^{2}:+\mathcal{I}_{g_{i_{1}} \bowtie g_{i_{2}}}^{2}+\frac{1}{2} \operatorname{tr} \bowtie\left(g_{i_{1}} \bowtie g_{i_{2}}\right) \mathcal{I}^{0}\right\}, \\
\mathcal{I}_{g_{1}}^{2} \mathcal{I}_{g_{2}}^{2} \mathcal{I}_{g_{3}}^{2}= & \sum_{\substack{i_{j}=1,2,3 \\
i_{1} \neq i_{2} \neq i_{3} \neq i_{1}}}\left\{\frac{1}{3 !}: \mathcal{I}_{g_{i_{1}}}^{2} \mathcal{I}_{g_{i_{2}}}^{2} \mathcal{I}_{g_{i_{3}}}^{2}:+:\left(\mathcal{I}_{g_{i_{1}} \bowtie g_{i_{2}}}^{2}+\frac{1}{2} \operatorname{tr} r_{\bowtie}\left(g_{i_{1}} \bowtie g_{i_{2}}\right) \mathcal{I}^{0}\right) \mathcal{I}_{g_{i_{3}}}^{2}:\right. \\
& \left.+\mathcal{I}_{g_{i_{1}} \bowtie g_{i_{2}} \bowtie g_{i_{3}}}^{2}+\frac{1}{3} \operatorname{tr} r_{\bowtie}\left(g_{i_{1}} \bowtie g_{i_{2}} \bowtie g_{i_{3}}\right) \mathcal{I}^{0}\right\} .
\end{aligned}
$$

As shown below, these formulae admit a natural generalization to the operator product of any number of multilinear currents $\mathcal{I}_{g}^{2 n}=: \underbrace{\mathcal{I}_{g}^{2} \cdots \mathcal{I}_{g}^{2}}_{n}$ :

$$
\mathcal{I}_{g_{1}}^{2 n_{1}} \ldots \mathcal{I}_{g_{k}}^{2 n_{k}}=\left.\left(\frac{\partial^{n_{1}}}{\left(\partial \mu^{1}\right)^{n_{1}}} \ldots \frac{\partial^{n_{k}}}{\left(\partial \mu^{k}\right)^{n_{k}}} E(\mathcal{V}(\mu))\right)\right|_{\mu=0}
$$

where

$$
\begin{gathered}
\mathcal{V}(\mu)=\sum_{j=1}^{\infty} \mu^{j} g_{j}, \\
E(\mathcal{V}(\mu))=\exp \left(-\operatorname{tr}_{\bowtie}\left(\ln _{\bowtie}\left(I d_{\bowtie}-\mathcal{V}(\mu)\right)\right) \exp _{\times}\left(\mathcal{G}(\mathcal{V}(\mu)) \bowtie\left(I d_{\bowtie}-\mathcal{G}(\mathcal{V}(\mu))\right)_{\bowtie}^{-1}\right),\right. \\
\mathcal{G}(g)=\mathcal{I}_{g}^{2}, \quad \mathcal{G}(g) \bowtie \mathcal{G}\left(g^{\prime}\right)=\mathcal{G}\left(g \bowtie g^{\prime}\right), \\
\ln _{\bowtie}\left(I d_{\bowtie}-\mathcal{V}(\mu)\right)=-\sum_{k=1}^{\infty} \frac{1}{k} \underbrace{\mathcal{V}(\mu) \bowtie \bowtie \mathcal{V}(\mu)}_{k}, \\
\exp _{\times}(A)=\sum_{n=0}^{\infty} \frac{1}{n !} \underbrace{A \times \ldots \times A}_{n}, \quad \underbrace{A \times \ldots \times A}_{0}=I d,
\end{gathered}
$$

where the commutative product $\times$ encodes the normal ordering

$$
\mathcal{I}_{g}^{2 n} \times \mathcal{I}_{f}^{2 m} \sim: \mathcal{I}_{g}^{2 n} \mathcal{I}_{f}^{2 m}:
$$

Using that $g_{i} \bowtie g_{i}=0$ and $\operatorname{tr}_{\bowtie}\left(g_{i}\right)=0$ by virtue of (6.37) and (6.39), Eq. (6.43) at $k=1$ implies that $\exp _{\times}(\mathcal{G}(\mu g))$ is the generating function for $2 n$-linear currents via

$$
\mathcal{I}_{g}^{2 n}=\left.\frac{\partial^{n}}{(\partial \mu)^{n}} \exp _{\times}(\mathcal{G}(\mu g))\right|_{\mu=0}
$$

Hence, Eqs. (6.43), (6.45) express the operator product of any number of multilinear currents via a linear combination of multilinear currents with composite arguments like in Eqs. (6.41), (6.42). 
Since $\times$ is associative and commutative, it is sometimes convenient to identify $\times$ with "usual" product. In this notation, formula (6.45) takes the form

$$
E(\mathcal{V}(\mu))=\operatorname{det}_{\bowtie}^{-1}\left(I d_{\bowtie}-\mathcal{V}(\mu)\right) \exp \left(\mathcal{G}(\mathcal{V}(\mu)) \bowtie\left(I d_{\bowtie}-\mathcal{G}(\mathcal{V}(\mu))\right)_{\bowtie}^{-1}\right),
$$

where

$$
\operatorname{det}_{\bowtie}\left(I d_{\bowtie}-\mathcal{V}(\mu)\right)=\exp \operatorname{tr}{ }_{\bowtie}\left(\ln _{\bowtie}\left(I d_{\bowtie}-\mathcal{V}(\mu)\right)\right) .
$$

By (6.45), the correlator of multilinear currents

$$
\Phi^{n_{1} \ldots n_{k}}\left(g_{1}, \ldots, g_{k}\right)=\left\langle\mathcal{I}_{g_{1}}^{2 n_{1}} \ldots \mathcal{I}_{g_{k}}^{2 n_{k}}\right\rangle
$$

is represented by the coefficient in front of the central element in the multiple product of multilinear currents, i.e.,

$$
\Phi^{n_{1} \ldots n_{k}}\left(g_{1}, \ldots g_{k}\right)=\left.\left(\frac{\partial^{n_{1}}}{\left(\partial \mu^{1}\right)^{n_{1}}} \ldots \frac{\partial^{n_{k}}}{\left(\partial \mu^{k}\right)^{n_{k}}} \operatorname{det}_{\bowtie}^{-1}\left|I d_{\bowtie}-\mathcal{V}(\mu)\right|\right)\right|_{\mu=0} .
$$

We call Eqs. 6.43) and (6.54) butterfly formulae. To obtain operator product and $n$-point equations for bilinear currents it remains to set $n_{1}=\ldots=n_{k}=1$.

Note that, as expected for Wightman functions, the result is not symmetric with respect to permutations of indices $1,2, \ldots k$ because butterfly product $\bowtie$ is sensitive to the ordering.

The direct proof of Eq. (6.43) is quite simple. As an illustration let us prove (6.43) for the product of bilinear currents. From (6.21) it follows

$$
\exp _{\times}\left(\mathcal{I}_{g}^{2}\right) \mathcal{I}_{f}^{2}=\exp _{\times}\left(\mathcal{I}_{g}^{2}\right) \times\left(\mathcal{I}_{f}^{2}+\mathcal{I}_{f \triangleright g+g \triangleleft f}^{2}+\mathcal{I}_{g \triangleleft f \triangleright g}^{2}+t r_{\triangleright}(g \triangleleft f) \mathcal{I}^{0}\right) .
$$

Substitution of $g=\sum_{k=1}^{\infty} \mathcal{V}_{k}(\mu)$ with $\mathcal{V}_{k}(\mu)=\underbrace{\mathcal{V}(\mu) \bowtie \ldots \bowtie \mathcal{V}(\mu)}_{k}$ into (6.55) gives

$$
\begin{gathered}
E(\mathcal{V}) \mathcal{I}_{f}^{2}=E(\mathcal{V}) \times\left(\mathcal{I}_{f}^{2}+\mathcal{I}_{f \triangleright \sum_{k=1}^{\infty} \mathcal{V}_{k}+\sum_{k=1}^{\infty} \mathcal{V}_{k} \triangleleft f}^{2}+\mathcal{I}_{\sum_{k=1}^{\infty}}^{\infty} \mathcal{V}_{k} \triangleleft f \triangleright \sum_{n=1}^{\infty} \mathcal{V}_{n}+\operatorname{tr}{ }_{\triangleright}\left(\sum_{k=1}^{\infty} \mathcal{V}_{k} \triangleleft f\right) \mathcal{I}^{0}\right) \\
=E(\mathcal{V}) \times\left(\mathcal{I}_{f}^{2}+\sum_{n \geq 1} \sum_{k=0}^{n} \mathcal{I}_{\mathcal{V}_{k} \triangleleft f \triangleright \mathcal{V}_{n-k}}^{2}+\operatorname{tr}_{\triangleright}\left(\sum_{k=1}^{\infty} \mathcal{V}_{k} \triangleleft f\right) \mathcal{I}^{0}\right) .
\end{gathered}
$$

On the other hand, direct differentiation gives

$$
\left.\frac{\partial}{\partial \mu^{i}} E(\mathcal{V}(\mu))\right|_{\mu^{i}=0}=E(\mathcal{V}(\mu)) \times\left.\left(\mathcal{I}_{g_{i}}^{2}+\sum_{k>1} \operatorname{tr}_{\bowtie}\left(\mathcal{V}_{k}(\mu) \bowtie g_{i}\right) \mathcal{I}^{0}+\sum_{n \geq 1} \sum_{k=0}^{n} \mathcal{I}_{\mathcal{V}_{k}(\mu) \bowtie g_{i} \bowtie \mathcal{V}_{n-k}(\mu)}^{2}\right)\right|_{\mu^{i}=0} .
$$

Let $i_{\max }$ be the maximal label among nonzero $\mu^{j}$. Comparison of $(6.56)$ with $f=g_{i_{\max }}$ and 6.57) at $i=i_{\max }$ gives

$$
\left.\frac{\partial}{\partial \mu^{i_{\max }}} E(\mathcal{V}(\mu))\right|_{\mu^{i_{\max }=0}}=E(\breve{\mathcal{V}}(\mu)) \mathcal{I}_{g_{i_{\max }}^{2}}, \quad \breve{\mathcal{V}}(\mu)=\left.\mathcal{V}(\mu)\right|_{\mu^{i_{\max }=0}}, \quad \breve{\mathcal{G}}(\mathcal{V}(\mu))=\left.\mathcal{G}(\mathcal{V}(\mu))\right|_{\mu^{i_{\max }=0}}
$$


because in this case $\breve{\mathcal{V}}_{k} \triangleleft f \triangleright \breve{\mathcal{V}}_{n-k}=\breve{\mathcal{V}}_{k} \bowtie f \bowtie \breve{V}_{n-k}$ and $\operatorname{tr}_{\triangleright}\left(\sum_{k=1}^{\infty} \breve{\mathcal{V}}_{k} \triangleleft f\right)=\operatorname{tr}{ }_{\bowtie}\left(\breve{\mathcal{V}}_{k}(\mu) \bowtie f\right)$. The inductional proof of $(6.43)$ is completed by observing that $\left.\partial_{\mu_{i}} E(\mathcal{V}(\mu))\right|_{\mu=0}=\mathcal{I}_{g_{i}}^{2}$. Since bilinear currents generate the full operator algebra this proves butterfly formula. An alternative derivation from the multiparticle algebra is presented in the next section.

Butterfly formulae encode information on all $n$-point functions and current operator algebra in an amazingly compact and efficient way as demonstrated in the sequel by practical computations. Concise and elegant form of butterfly formulae suggests that they should have some simple origin in a more general setup which is believed to result from a multiparticle HS theory. Appearance of the butterfly determinant indicates that it may admit some Gaussian (one-loop) representation. It is also worth to mention that the sum of all butterfly powers in the exponential in (6.45) describes all connected operator products.

\section{Butterfly formulae from multiparticle algebra}

\subsection{Multiple operator products from multiparticle algebra}

In [16] it was shown that the generating function for $2 n$-currents $\mathcal{I}_{g}^{2 n}(6.50)$, which are order- $n$ homogeneous functionals of $g$, has the form

$$
\begin{gathered}
\widetilde{G}_{g}=\operatorname{det}_{\star}^{-1}\left|e_{\star}-\Pi_{+} \star g\right| \exp \left(g \star\left(e_{\star}-\Pi_{+} \star g\right)_{\star}^{-1}\right), \\
\left(e_{\star}-A\right)_{\star}^{-1}=e_{\star}+A+\ldots+\underbrace{A \star A \star \ldots \star A}_{n}+\ldots .
\end{gathered}
$$

Note that in [16 notation $\nu$ was used instead of $g$ and the preexponential factor had power $\mathcal{N}$. The difference is that star product of 16] does not contain color indices, hence corresponding to the case of $\mathcal{N}=1$, while in this paper star product includes matrix multiplication over color indices. As a result, the trace of this paper is $\mathcal{N}$ times larger than that of [16], that, in accordance with (6.52), brings a power of $\mathcal{N}$ to all determinants. Taking this into account, the associative product, that reproduces the operator algebra of $A$-currents, is [16]

$$
\widetilde{G}_{g_{1}} \diamond \widetilde{G}_{g_{2}}=\frac{1}{\operatorname{det}_{\star}\left|\left(e_{\star}+\Pi_{+} \star \Upsilon_{2}\right)\right| \operatorname{det}_{\star}\left|e_{\star}-\Pi_{+} \star g_{1}\right| \operatorname{det}_{\star}\left|e_{\star}-\Pi_{+} \star g_{2}\right|} \widetilde{G}_{\mathbf{g}},
$$

where

$$
\begin{gathered}
\mathbf{g}=\left(\Upsilon_{2} \star\left(e_{\star}+\Pi_{+} \star \Upsilon_{2}\right)_{\star}^{-1}\right), \\
\Upsilon_{2}=\left(g_{1} \star\left(e_{\star}-\Pi_{+} \star g_{1}\right)_{\star}^{-1}+e_{\star}\right) \star\left(g_{2} \star\left(e_{\star}-\Pi_{+} \star g_{2}\right)_{\star}^{-1}+e_{\star}\right)-e_{\star} .
\end{gathered}
$$

By virtue of Eqs. (6.34), (6.37), in terms of butterfly product $\bowtie, \mathbf{g}(7.4)$ reads as

$$
\mathbf{g}=\left(e_{\bowtie}+g_{1}\right) \bowtie\left(e_{\bowtie}-g_{2} \bowtie g_{1}\right)_{\bowtie}^{-1} \bowtie g_{2}+\left(e_{\bowtie}+g_{2}\right) \bowtie\left(e_{\bowtie}-g_{1} \bowtie g_{2}\right)_{\bowtie}^{-1} \bowtie g_{1} .
$$

Using that the maps $f(g)=g \star\left(e_{\star}-\Pi_{+} g\right)_{\star}^{-1}$ and $f^{-1}(g)=g \star\left(e_{\star}+\Pi_{+} g\right)_{\star}^{-1}$ are mutually inverse, i.e., $f^{-1}(f(g))=g$, it is easy to obtain the composition law for the product of any

number $n$ of generating functions that encodes the operator product of any number of operators

$$
\widetilde{G}_{g_{1}} \diamond \ldots \diamond \widetilde{G}_{g_{n}}=\frac{\tilde{\eta}\left(g_{1}\right) \ldots \tilde{\eta}\left(g_{n}\right)}{\tilde{\eta}\left(\Upsilon_{n} \star\left(e_{\star}+\Pi_{+} \star \Upsilon_{n}\right)_{\star}^{-1}\right)} \widetilde{G}_{\mathbf{g}}
$$


where

$$
\begin{array}{rlrl}
\tilde{\eta}(A) & =\exp \left[-t r_{\star}\left(\log _{\star}\left(e_{\star}-\Pi_{+} \star A\right)\right)\right], & & \mathbf{g}=\left(\Upsilon_{n} \star\left(e_{\star}+\Pi_{+} \star \Upsilon_{n}\right)_{\star}^{-1}\right), \\
\Upsilon_{n}=\left(\tilde{g}_{1}+e_{\star}\right) \star \ldots \star\left(\tilde{g}_{n}+e_{\star}\right)-e_{\star}, & \tilde{g}_{i}=g_{i} \star\left(e_{\star}-\Pi_{+} \star g_{i}\right)_{\star}^{-1} .
\end{array}
$$

In these terms, the operator product $\mathcal{I}_{g_{1}}^{2} \ldots \mathcal{I}_{g_{n}}^{2}$ is described by the part of $(7.7)$ polylinear in $g_{1}, \ldots, g_{n}$. OPE of multilinear currents $\mathcal{I}_{g_{i}}^{2 n_{i}}$ are associated with the terms of order $n_{i}$ in $g_{i}$.

To obtain from here butterfly formulae it is convenient to use notations (6.35), rewriting (7.7) in the form

$$
\widetilde{G}_{\tilde{g}_{1}} \diamond \ldots \diamond \widetilde{G}_{\tilde{g}_{n}}=\exp \left[\sum_{m \leq n} \operatorname{tr} \underset{\triangleright}{ }\left(\log _{\triangleright}\left(e_{\triangleright}+\tilde{g}_{m}\right)\right)+\operatorname{tr} \underset{\bowtie}{ }\left(\log _{\triangleright}\left(e_{\triangleright}-\Upsilon_{n}\right)\right)\right] \widetilde{G}_{\mathbf{g}}
$$

with

$$
\mathbf{g}=\Upsilon_{n} \stackrel{\triangleright}{\bowtie}\left(e_{\triangleright}-\Upsilon_{n}\right)_{\triangleright}^{-1} .
$$

$\Upsilon_{n}(7.9)$ can be understood as

$$
\Upsilon_{n}=\left.\Upsilon\right|_{g_{i>n}=0}
$$

where

$$
\Upsilon=\sum_{\substack{k=1 \\ i_{1}<\ldots<i_{k}}}^{\infty} \tilde{g}_{i_{1}} \star \ldots \star \tilde{g}_{i_{k}} \quad \text { with } \quad \tilde{g}_{i}=\sum_{k \geq 0} \underbrace{g_{i} \bowtie \triangleright^{\triangleright} \ldots g_{i}}_{k} .
$$

Let

$$
\mathcal{S}=\sum_{i=1}^{\infty} \tilde{g}_{i}
$$

Butterfly formulae follow from $(7.10)$ by virtue of the following fundamental relations proven in Section 7.2

$$
\begin{aligned}
& \mathbf{g}=\Upsilon \stackrel{\triangleright}{\bowtie}\left(e_{\triangleright}-\Upsilon\right)_{\triangleright}^{-1}=\left(e_{\unrhd}-\mathcal{S}\right)_{\unrhd}^{-1} \unrhd \mathcal{S}, \\
& \operatorname{tr} r_{\bowtie}\left(\log _{\triangleright}\left(e_{\triangleright}-\Upsilon\right)\right)=\operatorname{tr} r_{\bowtie}\left(\log _{\unrhd}\left(e_{\bowtie}-\mathcal{S}\right)\right) .
\end{aligned}
$$

By virtue of (7.15), (7.16), Eqs. (7.10), (7.11) give the generating formula (6.43) with

$$
\begin{aligned}
& E(\widetilde{\mathcal{V}}(\mu))=\exp \left(-\operatorname{tr}_{\unrhd}\left(\ln _{\unrhd}\left(I d_{\bowtie}-\widetilde{\mathcal{V}}(\mu)\right)\right)\right) \exp _{\times}\left(\mathcal{G}(\widetilde{\mathcal{V}}(\mu)) \unrhd\left(I d_{\bowtie}-\mathcal{G}(\widetilde{\mathcal{V}}(\mu))\right)_{\unrhd}^{-1}\right), \\
& \widetilde{\mathcal{V}}(\mu)=\sum_{j=1}^{\infty} \tilde{g}_{j}\left(\mu^{j}\right), \quad \tilde{g}_{i}\left(\mu^{i}\right)=\mu^{i} g_{i} \unrhd\left(e_{凶}-\mu^{i} g_{i}\right)_{\unrhd}^{-1}, \\
& \mathcal{G}(\widetilde{\mathcal{V}}(\mu)) \bowtie \mathcal{G}\left(\widetilde{\mathcal{V}}^{\prime}(\mu)\right)=\mathcal{G}\left(\widetilde{\mathcal{V}}(\mu) \bowtie \widetilde{\mathcal{V}}^{\prime}(\mu)\right) .
\end{aligned}
$$

The remarkable property of equation (7.17) is that multiple $₫$ products of $g_{i}$ in (7.18) cancel against those resulting from the butterfly product $₫$ in (7.17) in such a way that no term containing $g_{j} \bowtie g_{j}$ survives. This result is anticipated because the terms with the same 
$g_{i}$ are associated with some $\mathcal{I}_{g_{i}}^{2 n}$ which is normal-ordered, and hence never undergo a Wick contraction giving rise to $\bowtie$ products of $g_{i}$. The simplest way to see how this cancellation occurs is to use formula (7.6) which shows that this is indeed true for the product of a pair of multilinear currents. By induction it remains true for the product of any number of multiparticle currents.

Effectively, this allows us to replace $\tilde{g}_{i}(7.18)$ by $g_{i}$ and the product $₫$ by $\bowtie$ in $(7.17)$ to obtain formula (6.45).

\subsection{Proof of fundamental relations}

The proof of (7.15) and (7.16) is based on the mutual associativity of the involved butterfly products. Let us first prove relation (7.15).

I. Using that $\bar{\bowtie}=(\bowtie-\bowtie)$, Eq. (7.15) can be equivalently rewritten as

$$
\Upsilon=\mathcal{S}+\mathcal{S} \bar{\bowtie} \Upsilon
$$

With the help of 6.35$)$ we obtain

$$
\mathcal{S} \bowtie \sum_{\substack{1 \leq k \leq n \\ i_{1}<\cdots<i_{k} \leq n}} \tilde{g}_{i_{1}} \star \ldots \star \tilde{g}_{i_{k}}=\sum_{\substack{2 \leq k \leq n \\ i_{1}<\ldots<i_{k} \leq n}} \tilde{g}_{i_{1}} \star \ldots \star \tilde{g}_{i_{k}},
$$

which by virtue of (7.13) gives (7.20), thus proving (7.15).

Note that the identity (7.20) can be equivalently written in the form

$$
\left(e_{\bar{\bowtie}}-\mathcal{S}\right) \bar{\bowtie}\left(e_{\bar{\bowtie}}+\Upsilon\right)=e_{\bar{\bowtie}} .
$$

From where it follows in particular that $\mathcal{S} \bowtie \Upsilon=\Upsilon \bar{凶} \mathcal{S}$.

II. Let us first prove the determinant relation (7.16) directly by checking its variation with respect to $g_{i}$. Practically, it is convenient to proceed by induction first checking that the variation $\delta_{1}$ with respect to $\delta g_{1}$ gives identity

$$
\delta_{1} \operatorname{tr} \underset{\bowtie}{ }\left(\log _{\triangleright}\left(e_{\triangleright}-\Upsilon\right)\right)=\delta_{1} t r_{\unrhd}\left(\log _{\unrhd}\left(e_{\bowtie}-\mathcal{S}\right)\right) .
$$

After this is checked, one can set $g_{1}=0$ and prove analogously that the variation with respect to $g_{2}$ gives identity, etc.

By virtue of (7.15), (7.23) is equivalent to

$$
\delta_{1} \operatorname{tr} \underset{\bowtie}{ }\left(\log _{\triangleright}\left(e_{\triangleright}-\Upsilon\right)\right)=-t r_{\bowtie}\left(\delta_{1}(\mathcal{S})+\delta_{1}(\mathcal{S}) \unrhd \Upsilon \bowtie\left(e_{\triangleright}-\Upsilon\right)_{\triangleright}^{-1}\right) .
$$

On the other hand,

$$
\delta_{1} \operatorname{tr} \underset{\triangleright}{ }\left(\log _{\triangleright}\left(e_{\triangleright}-\Upsilon\right)\right)=-t r_{\triangleright}\left(\delta \tilde{g}_{1} \star\left(\Upsilon^{\prime}+e_{\star}\right) \stackrel{\triangleright}{\triangleright}\left(e_{\triangleright}-\Upsilon\right)_{\triangleright}^{-1}\right),
$$


where

$$
\Upsilon^{\prime}=\left(\tilde{g}_{2}+e_{\star}\right) \star \ldots \star\left(\tilde{g}_{n}+e_{\star}\right)-e_{\star} .
$$

By virtue of (6.31) and (7.25)

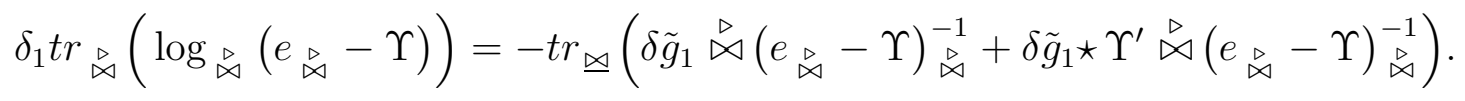

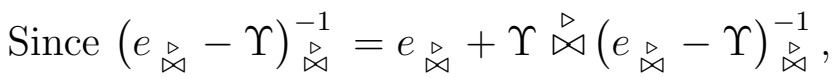

$$
\delta_{1} t r_{\triangleright}\left(\log _{\triangleright}\left(e_{\triangleright}-\Upsilon\right)\right)=-t r_{\unrhd}\left(\delta \tilde{g}_{1}+\delta \tilde{g}_{1}\left(\star \Upsilon^{\prime}+\stackrel{\triangleright}{\bowtie}\right) \stackrel{\triangleright}{\bowtie}\left(e_{\triangleright}-\Upsilon\right)_{\triangleright}^{-1}\right) .
$$

By virtue of (6.36), this proves (7.24) using that $\delta_{1}(\mathcal{S})=\delta \tilde{g}_{1}$.

III. Determinant relation (7.16) is a particular case of a general relation not referring to the detailed structure of the operators $\Upsilon$ and $\mathcal{S}$. Namely, let $A$ be a biassociative algebra with the mutually associative product laws $\bullet$ and $\diamond$ and respective traces obeying

$$
\operatorname{tr}_{\diamond}(a \bullet b)=\operatorname{tr}_{\bullet}(b \diamond a), \quad \forall a, b \in A .
$$

Let

$$
\diamond=\bullet-\circ .
$$

Then

$$
t_{\bullet}\left(\log _{\bullet}\left(e_{\bullet}+a \diamond\left(e_{\diamond}-a\right)_{\diamond}^{-1}\right)-t r_{\diamond}\left(\log _{\diamond}\left(e_{\diamond}+a\right)\right)+t r_{\diamond}\left(\log _{\diamond}\left(e_{\diamond}-a\right)\right)=0,\right.
$$

or, equivalently,

$$
\operatorname{det}_{\bullet}\left(e_{\bullet}+a \diamond\left(e_{\diamond}-a\right)_{\diamond}^{-1}\right) \operatorname{det}_{\diamond}\left(e_{\diamond}-a\right)=\operatorname{det}_{\diamond}\left(e_{\diamond}+a\right) .
$$

This gives (7.16) upon identification of $\bowtie, \bowtie$ and $\bar{\bowtie}$ with $\bullet, \circ$ and $\diamond$, respectively, taking into account that $\operatorname{det}_{\diamond}\left(e_{\diamond}-a\right)=1$ because

$$
\operatorname{tr}_{\bar{\bowtie}} \underbrace{(\mathcal{S} \bowtie \ldots \bar{\Phi} \mathcal{S})}_{n}=0 \text { at } n>0 .
$$

To prove (7.31) it is convenient to introduce the product

$$
\stackrel{t}{\circ}=\circ+t \diamond, \quad t \in[0,1],
$$

obeying

For any $a_{0} \in A$,

$$
\frac{\partial}{\partial t} \stackrel{t}{\circ}=\diamond, \quad \stackrel{0}{\circ}=\circ, \quad \stackrel{1}{\circ}=\bullet .
$$

$$
a_{t}=a_{0} \diamond\left(e_{\diamond}-t a_{0}\right)_{\diamond}^{-1}
$$

obeys

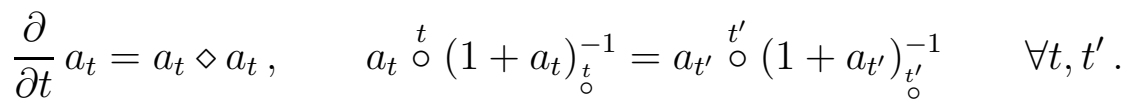

From (7.29), (7.35) and (7.37) it follows that

$$
\frac{\partial}{\partial t} \operatorname{tr}_{\mathrm{o}}\left(\log _{\mathrm{o}}\left(e_{\mathrm{o}}+a_{t}\right)\right)=t r_{\diamond}\left(a_{t}\right) \text {. }
$$

With the help of (7.36), integration of this relation gives (7.31). 


\section{Space-time current operator algebra}

Once current operator algebra in the twistor space is known, it is straightforward to extend it to space-time. Indeed, unfolded form of the current equation (2.17) uniquely determines dependence on space-time coordinates in terms of twistor coordinates by virtue of (2.22) or (3.14). It is important that the latter formulae hold for general conserved currents, i.e., not necessarily built from free fields. This is because the unfolded current equation holds independently of the origin of conformal conserved currents.

More generally, this implies that, in the framework of unfolded formulation, operator algebra is fully determined by the associative operator algebra of functions of generalized twistor variables of the system in question. Analysis and classification of twistor algebras is much simpler than that of space-time operator algebras. As shown in the previous section, for free massless fields, this algebra is based on the star-product HS algebra. More precisely, twistor operator algebra is the universal enveloping algebra of the HS algebra (for more detail see [16]). This simple observation clarifies in particular the difference between $2 d$ and $3 d$ conformal theories pointed out in [22, 23]: $3 d$ conformal HS algebras admit no deformation, while $2 d$ conformal HS algebras belong to a parametric family of algebras described in particular in [41] (and references therein).

\subsection{A-currents}

Space-time operator algebra is the algebra of currents $\mathrm{J}_{\gamma}\left(Y_{1}, Y_{2} \mid X\right)(4.27)$ at $Y_{1}=Y_{2}=0$ with parameters $\gamma=\gamma_{a b}^{m n}(\mathcal{A})$, that depend on $\mathcal{A}_{ \pm}(2.29$ ) and obey (4.32),

$$
\mathrm{J}_{\gamma}(X)=\left.\mathrm{J}_{\gamma}\left(Y_{1}, Y_{2} \mid X\right)\right|_{Y_{1,2}=0} .
$$

It should be stressed that setting to zero $Y$-variables does not imply any loss of generality since the dependence of currents on twistor variables is fully accounted by the freedom in the parameters $\gamma$. To compute operator algebra for all conformal currents and their descendants it suffices to consider parameters $\gamma_{a b}^{m n}\left(\mathcal{A}_{+}\left(Y_{1} \mid X\right), \mathcal{A}_{-}\left(Y_{2} \mid X\right)\right)$ that only depend on the operators $\mathcal{A}_{ \pm C}(2.29)$ which represent $Y$-derivatives. However, in most of our consideration, we keep the dependence on the $X$-dependent operators $\mathcal{A}_{ \pm}^{C}$ since this does not affect the analysis too much.

A useful trick is to interpret $\mathrm{J}_{\gamma}\left(Y_{1}, Y_{2} \mid X\right)$ (4.27) as the current $\mathcal{I}_{g}^{2}$ (6.1) with a function $g(W)=\mathfrak{g}\left(Y_{1}, Y_{2}, X ; \gamma \mid W\right)$ that depends on $Y_{i}, X$ and $\gamma$ as parameters

$$
\mathrm{J}_{\gamma}\left(Y_{1}, Y_{2} \mid X\right)=\mathcal{I}_{\mathfrak{g}\left(Y_{1}, Y_{2}, X ; \gamma\right)}^{2}
$$

To find $\mathfrak{g}\left(Y_{1}, Y_{2}, X ; \gamma\right)$ we observe that, due to evolution formula (3.14) along with the following identity for $C_{m}^{a}(Y \mid X)$ of definite parity

$$
\int d^{M} W \mathcal{D}_{a}(W-Y \mid X) C_{m}^{a}(W \mid 0)=(-1)^{\mathfrak{p}_{m}} \int d^{M} W \mathcal{D}_{a}(W+Y \mid X) C_{m}^{a}(W \mid 0)
$$


(no summation over $a$ ), $\mathrm{J}_{\gamma}(4.28)$ acquires the form

$\mathrm{J}_{\gamma}\left(Y_{1}, Y_{2} \mid X\right)=-\sum_{m, n, a, b} \gamma_{a b}^{m n}\left(\mathcal{A}_{+}\left(Y_{1} \mid X\right), \mathcal{A}_{-}\left(Y_{2} \mid X\right)\right) \int d W_{1,2} \mathfrak{D}_{a b}^{\mathfrak{p}_{m} \mathfrak{p}_{n}}\left(Y_{1}, Y_{2}, X \mid W_{1}, W_{2}\right) \hat{\mathrm{T}}_{m n}^{a b}\left(W_{1}, W_{2} \mid 0\right)$

with $a, b=+,-, \hat{\mathrm{T}}_{j k}^{a b}(4.8$,

$$
\mathfrak{D}_{a b}^{\mathfrak{p}_{m} \mathfrak{p}_{n}}\left(Y_{1}, Y_{2}, X \mid W_{1}, W_{2}\right)=\left(\kappa_{1}^{a}\right)^{\mathfrak{p}_{m}}\left(\kappa_{2}^{b}\right)^{\mathfrak{p}_{n}} \mathcal{D}_{a}^{\mathfrak{p}_{m}}\left(W_{1}, \kappa_{1}^{a} Y_{1} \mid-X\right) \mathcal{D}_{b}^{\mathfrak{p}_{n}}\left(W_{2}, \kappa_{2}^{b} Y_{2} \mid-X\right),
$$

$\kappa_{j}^{a}(4.9)$ and

$$
\mathcal{D}_{a}^{\mathfrak{p}_{m}}(W, Y \mid X)=\mathcal{D}_{a}^{\mathfrak{p}_{m}}(Y, W \mid X)=\frac{1}{2}\left(\mathcal{D}_{a}(W-Y \mid X)+(-1)^{\mathfrak{p}_{m}} \mathcal{D}_{a}(W+Y \mid X)\right) .
$$

By virtue of (3.10), (3.15) and (3.17), for non-degenerate $X$,

$$
\mathcal{D}_{-}^{\mathfrak{p}_{k}}(W, Y \mid X)=\mathcal{D}_{-}(X) \mathcal{E}^{\mathfrak{p}_{k}}(W, Y \mid X), \quad \mathcal{D}_{+}^{\mathfrak{p}_{k}}(i W, i Y \mid X)=\mathcal{D}_{+}(X) \mathcal{E}^{\mathfrak{p}_{k}}(W, Y \mid X)
$$

where

$$
\begin{gathered}
\mathcal{D}_{ \pm}(X)=-\frac{i}{2^{M} \pi^{\frac{M}{2}}} \exp \left( \pm \frac{i \pi I_{X}}{4}\right) \frac{1}{\sqrt{|\operatorname{det}(X)|}} \\
\mathcal{E}^{0}(W, Y \mid X)=\exp \left(-\frac{i}{4} X_{A B}^{-1}(W W+Y Y)^{A B}\right) \cos \left(\frac{1}{2} X_{A B}^{-1} W^{A} Y^{B}\right)
\end{gathered}
$$

and

$$
\mathcal{E}^{1}(W, Y \mid X)=i \exp \left(-\frac{i}{4} X_{A B}^{-1}(W W+Y Y)^{A B}\right) \sin \left(\frac{1}{2} X_{A B}^{-1} W^{A} Y^{B}\right) .
$$

$\mu$-symmetrization of (8.4) with $\mu(6.3)$ gives

$$
\mathrm{J}_{\gamma}\left(Y_{1}, Y_{2} \mid X\right)=-\sum_{a, b} \int d W_{1} d W_{2} \mathfrak{g}_{a b}^{m n}\left(Y_{1}, Y_{2}, X ; \gamma \mid W_{1}, W_{2}\right) \hat{\mathrm{T}}_{m n}^{a b}\left(W_{1}, W_{2} \mid 0\right)
$$

where

$\mathfrak{g}_{a b}^{m n}\left(Y_{1}, Y_{2}, X ; \gamma \mid W_{1}, W_{2}\right)=-\frac{1}{2}(1+\mu)\left(\gamma_{a b}^{m n}\left(\mathcal{A}_{+}\left(Y_{1} \mid X\right), \mathcal{A}_{-}\left(Y_{2} \mid X\right)\right) \mathfrak{D}_{a b}^{\mathfrak{p}_{m} \mathfrak{p}_{n}}\left(Y_{1}, Y_{2}, X \mid W_{1}, W_{2}\right)\right)$

that by construction satisfies (6.2) as well as (6.4) by virtue of (4.32).

Using butterfly formula (6.43), Eq. (8.2) determines the operator product of any number of multilinear space-time currents $\mathrm{J}_{\gamma}^{2 k}=: \underbrace{\mathrm{J}_{\gamma} \ldots \mathrm{J}_{\gamma}}_{k}$ :

$$
\mathrm{J}_{\gamma_{1}}^{2 k_{1}}\left(Y_{1}^{1}, Y_{2}^{1} \mid X^{1}\right) \ldots \mathrm{J}_{\gamma_{n}}^{2 k_{n}}\left(Y_{1}^{n}, Y_{2}^{n} \mid X^{n}\right)=\left.\left(\frac{\partial^{k_{1}}}{\left(\partial \mu^{1}\right)^{k_{1}}} \ldots \frac{\partial^{k_{n}}}{\left(\partial \mu^{n}\right)^{k_{n}}} E(\mathfrak{V}(\mu))\right)\right|_{\mu=0}
$$


where

$$
\begin{gathered}
\mathfrak{V}(\mu)=\sum_{j=1}^{\infty} \mu^{j} g_{j}, \quad g_{j}=\mathfrak{g}\left(Y_{1}^{j}, Y_{2}^{j}, X^{j} ; \gamma_{j}\right), \\
E(\mathfrak{V}(\mu))=\exp \left[-t r_{\bowtie}\left(\ln _{\bowtie}\left(I d_{\bowtie}-\mathfrak{V}(\mu)\right)\right] \exp _{\times}\left(\mathcal{G}(\mathfrak{V}(\mu)) \bowtie\left(I d_{\bowtie}-\mathcal{G}(\mathfrak{V}(\mu))\right)_{\bowtie}^{-1}\right)\right.
\end{gathered}
$$

with $\mathcal{G}(g)=\mathcal{I}_{g}^{2}$. The product of bilinear currents is obtained at $k_{1}=\ldots=k_{n}=1$.

Operator product of space-time multilinear currents results from (8.13) at $Y=0$

$$
\mathrm{J}_{\gamma_{1}}^{2 k_{1}}\left(X^{1}\right) \ldots \mathrm{J}_{\gamma_{n}}^{2 k_{n}}\left(X^{n}\right)=\left.\mathrm{J}_{\gamma_{1}}^{2 k_{1}}\left(Y_{1}^{1}, Y_{2}^{1} \mid X^{1}\right) \ldots \mathrm{J}_{\gamma_{n}}^{2 k_{n}}\left(Y_{1}^{n}, Y_{2}^{n} \mid X^{n}\right)\right|_{Y=0}
$$

In this case, formulae are simplified further due to simplification of $\left.\mathfrak{g}_{a b}^{m n}\left(Y_{1}, Y_{2}, X ; \gamma \mid W_{1}, W_{2}\right)\right|_{Y=0}$ on the r.h.s. of (8.13), that only depends on $\rho$-invariant parameters $\gamma_{a b}^{+m n}$, i.e.,

$$
\mathfrak{g}_{a b}^{0 m n}\left(X ; \gamma \mid W_{1}, W_{2}\right):=\left.\mathfrak{g}_{a b}^{m n}\left(Y_{1}, Y_{2}, X ; \gamma \mid W_{1}, W_{2}\right)\right|_{Y=0}=\left.\mathfrak{g}_{a b}^{m n}\left(Y_{1}, Y_{2}, X ; \gamma^{+} \mid W_{1}, W_{2}\right)\right|_{Y=0} .
$$

In fact, this is anticipated as a consequence of (4.14). More formally, we observe that, by virtue of (4.11),

$$
\left(\kappa_{1}^{a}\right)^{\mathfrak{p}_{m}} \mathcal{D}_{a}^{\mathfrak{p}_{m}}\left(W, \kappa_{1}^{a} Y \mid X\right)=(i)^{\mathfrak{p}_{m}}\left(\kappa_{2}^{a}\right)^{\mathfrak{p}_{m}} \mathcal{D}_{a}^{\mathfrak{p}_{m}}\left(W, i \kappa_{2}^{a} Y \mid X\right) .
$$

Hence

$$
\mathfrak{D}_{a b}^{\mathfrak{p}_{m} \mathfrak{p}_{n}}\left(Y_{1}, Y_{2}, X \mid W_{1}, W_{2}\right)=i^{\mathfrak{p}_{m}+\mathfrak{p}_{n}} \mathfrak{D}_{b a}^{\mathfrak{p}_{n} \mathfrak{p}_{m}}\left(i Y_{2}, i Y_{1}, X \mid W_{2}, W_{1}\right)
$$

and

$$
\mu\left(\mathfrak{D}_{a b}^{\mathfrak{p}_{m} \mathfrak{p}_{n}}\left(Y_{1}, Y_{2}, X \mid W_{1}, W_{2}\right)\right)=i^{\mathfrak{p}_{m, n}} \mathfrak{D}_{b a}^{\mathfrak{p}_{n} \mathfrak{p}_{m}}\left(i Y_{2}, i Y_{1}, X \mid W_{1}, W_{2}\right) .
$$

Since, by virtue of (2.30), $\gamma^{ \pm}$(4.31) satisfy

$$
\gamma_{a b}^{ \pm m n}\left(\mathcal{A}_{+}\left(Y_{1} \mid X\right), \mathcal{A}_{-}\left(Y_{2} \mid X\right)\right)= \pm(-i)^{\mathfrak{p}_{m}+\mathfrak{p}_{n}} \gamma_{b a}^{ \pm n m}\left(\mathcal{A}_{+}\left(i Y_{2} \mid X\right), \mathcal{A}_{-}\left(i Y_{1} \mid X\right)\right)
$$

Eq. (8.12) can be written in the form

$$
\begin{array}{r}
\mathfrak{g}_{a b}^{m n}\left(Y_{1}, Y_{2}, X ; \gamma \mid W_{1}, W_{2}\right)=-\frac{1}{2}\left\{\left(\gamma_{a b}^{+m n}+\gamma_{a b}^{-m n}\right)\left(\mathcal{A}_{+}\left(Y_{1} \mid X\right), \mathcal{A}_{-}\left(Y_{2} \mid X\right)\right) \mathfrak{D}_{a b}^{\mathfrak{p}_{m} \mathfrak{p}_{n}}\left(Y_{1}, Y_{2}, X \mid W_{1}, W_{2}\right)\right. \\
\left.+\left(\gamma_{a b}^{+m n}-\gamma_{a b}^{-m n}\right)\left(\mathcal{A}_{+}\left(i Y_{2} \mid X\right), \mathcal{A}_{-}\left(i Y_{1} \mid X\right)\right) \mathfrak{D}_{a b}^{\mathfrak{p}_{m} \mathfrak{p}_{n}}\left(i Y_{2}, i Y_{1}, X \mid W_{1}, W_{2}\right)\right\} .
\end{array}
$$

To prove (8.12) it remains to observe that $\gamma^{-}$-dependent terms cancel at $Y_{1,2}=0$.

Since, by virtue of $(6.8),(6.9)$, butterfly product $\mathcal{G}(\mathfrak{g}) \bowtie \mathcal{G}\left(\mathfrak{g}^{\prime}\right)(6.46)$ deals with integrations of $\mathfrak{g}, \mathfrak{g}^{\prime}$ over variables $W$, that does not involve variables $Y$, Eq. (8.13) along with (8.16), (8.17) gives

$$
\mathrm{J}_{\gamma_{1}}^{2 k_{1}}\left(X^{1}\right) \ldots \mathrm{J}_{\gamma_{n}}^{2 k_{n}}\left(X^{n}\right)=\left.\left(\frac{\partial^{k_{1}}}{\left(\partial \mu^{1}\right)^{k_{1}}} \ldots \frac{\partial^{k_{n}}}{\left(\partial \mu^{n}\right)^{k_{n}}} E\left(\mathfrak{V}^{0}(\mu)\right)\right)\right|_{\mu=0}
$$

where

$$
\mathfrak{V}^{0}(\mu)=\sum_{j=1}^{\infty} \mu^{j} g_{j}^{0}, \quad g_{j}^{0}=\mathfrak{g}^{0}\left(X^{j} ; \gamma_{j}\right)
$$




$$
\mathfrak{g}_{a b}^{0 m n}\left(X ; \gamma \mid W_{1}, W_{2}\right)=-\left.\gamma^{+m n}{ }_{a b}^{m n}\left(\mathcal{A}_{+}\left(Y_{1} \mid X\right), \mathcal{A}_{-}\left(Y_{2} \mid X\right)\right) \mathfrak{D}_{a b}^{\mathfrak{p}_{m} \mathfrak{p}_{n}}\left(Y_{1}, Y_{2}, X \mid W_{1}, W_{2}\right)\right|_{Y=0} .
$$

According to (6.37)

$$
\mathfrak{g}^{0}\left(X^{i} ; \gamma_{i}\right) \bowtie \mathfrak{g}^{0}\left(X^{j} ; \gamma_{j}\right)=\theta(j-i) \mathfrak{g}^{0}\left(X^{i} ; \gamma_{i}\right) \triangleleft \mathfrak{g}^{0}\left(X^{j} ; \gamma_{j}\right)+\theta(i-j) \mathfrak{g}^{0}\left(X^{i} ; \gamma_{i}\right) \triangleright \mathfrak{g}^{0}\left(X^{j} ; \gamma_{j}\right)
$$

where Eqs. (6.8), (6.9), (8.5) and (8.25) along with the following consequence of Eq. (3.16)

$$
\int d^{M} p \mathcal{D}_{\mp}^{\mathfrak{p}_{k}}(p, Y \mid X) \mathcal{D}_{ \pm}^{\mathfrak{p}_{l}}\left(p, Y^{\prime} \mid X^{\prime}\right)=-i \delta^{\mathfrak{p}_{k} \mathfrak{p}_{l}} \mathcal{D}_{\mp}^{\mathfrak{p}_{k}}\left(Y, Y^{\prime} \mid X-X^{\prime}\right)
$$

give

$$
\begin{aligned}
& \left(\mathfrak{g}^{0}\left(X^{q} ; \gamma_{q}\right) \triangleleft \mathfrak{g}^{0}\left(X^{p} ; \gamma_{p}\right)\right)_{a d}^{m l}\left(W_{1}, W_{2}\right) \\
= & -2 i \delta_{n k}\left(\left(\gamma_{q}^{+}\right)_{a-}^{m n}\left(\mathcal{A}_{+}\left(Y_{1}^{q} \mid X^{q}\right), \mathcal{A}_{-}\left(Y_{2}^{q} \mid X^{q}\right)\right)\left(\gamma_{p}^{+}\right)_{+d}^{k l}\left(\mathcal{A}_{+}\left(Y_{1}^{p} \mid X^{p}\right), \mathcal{A}_{-}\left(Y_{2}^{p} \mid X^{p}\right)\right)\right. \\
& \left.\delta^{\mathfrak{p}_{q}^{2} \mathfrak{p}_{p}^{1}} \mathfrak{D}_{a d}^{\mathfrak{p}_{m} \mathfrak{p}_{l}}\left(Y_{1}^{q}, Y_{2}^{p}, X^{q}, X^{p} \mid W_{1}, W_{2}\right) \mathcal{D}_{-}^{\mathfrak{p}_{q}^{2}}\left(Y_{2}^{q}, Y_{1}^{p} \mid X^{p}-X^{q}\right)\right)\left.\right|_{Y=0}, \\
=\quad & -2 i \delta_{n k}\left(\left(\gamma_{q}^{+}\right)_{a+}^{m n}\left(\mathcal{A}_{+}\left(Y_{1}^{q} \mid X^{q}\right), \mathcal{A}_{-}\left(Y_{2}^{q} \mid X^{q}\right)\right)\left(\gamma_{p}^{+}\right)_{-d}^{k l}\left(\mathcal{A}_{+}\left(Y_{1}^{p} \mid X^{p}\right), \mathcal{A}_{-}\left(Y_{2}^{p} \mid X^{p}\right)\right)\right. \\
& \left.\delta^{\mathfrak{p}_{q}^{2} \mathfrak{p}_{p}^{1}} \mathfrak{D}_{a d}^{\mathfrak{p}_{m} \mathfrak{p}_{l}}\left(Y_{1}^{q}, Y_{2}^{p}, X^{q}, X^{p} \mid W_{1}, W_{2}\right) \mathcal{D}_{+}^{\mathfrak{p}_{q}^{2}}\left(i Y_{2}^{q}, i Y_{1}^{p} \mid X^{p}-X^{q}\right)\right)\left.\right|_{Y=0},
\end{aligned}
$$

where

$$
\mathfrak{D}_{a b}^{\mathfrak{p}_{m} \mathfrak{p}_{n}}\left(Y_{1}, Y_{2}, X_{1}, X_{2} \mid W_{1}, W_{2}\right)=\left(\kappa_{1}^{a}\right)^{\mathfrak{p}_{m}}\left(\kappa_{2}^{b}\right)^{\mathfrak{p}_{n}} \mathcal{D}_{a}^{\mathfrak{p}_{m}}\left(W_{1}, \kappa_{1}^{a} Y_{1} \mid-X_{1}\right) \mathcal{D}_{b}^{\mathfrak{p}_{n}}\left(W_{2}, \kappa_{2}^{b} Y_{2} \mid-X_{2}\right)
$$

and we assume that $\gamma_{j}=\gamma_{j} \underset{a_{j} b_{j}}{m_{j} n_{j}}$ has definite parities with respect to the first and second arguments

$$
\mathfrak{p}_{j}^{1}:=\mathfrak{p}_{m_{j}}, \quad \mathfrak{p}_{j}^{2}:=\mathfrak{p}_{n_{j}} \quad \forall m_{j}, n_{j} .
$$

Recall, that additional factors of $i$ in $\mathcal{D}_{ \pm}^{\mathfrak{p}_{k}}$ of (8.29) result from Eqs. (4.9) and (8.5).

To obtain explicit formulae for $g_{1}^{0} \bowtie \ldots \bowtie g_{n}^{0}$ and $\operatorname{tr}_{\bowtie}\left(g_{1}^{0} \bowtie \ldots \bowtie g_{n}^{0}\right)$ with $g_{j}^{0}$ (8.24), we introduce butterfly product of parameters $\gamma_{i}=\gamma_{i}\left(\mathcal{A}_{+}\left(Y_{1}^{i} \mid X^{i}\right), \mathcal{A}_{-}\left(Y_{2}^{i} \mid X^{i}\right)\right)$ as follows

$$
\left(\gamma_{i, j}\right)_{a d}^{m l}=\left(\gamma_{i} \bowtie \gamma_{j}\right)_{a d}^{m l}=\delta_{n k}\left\{\theta(j-i) \tau^{b c}+\theta(i-j) \tau^{c b}\right\}\left(\gamma_{i}\right)_{a b}^{m n}\left(\gamma_{j}\right)_{c d}^{k l}
$$

and, inductively, $\gamma_{j_{1}, \ldots, j_{p}}=\gamma_{j_{1}} \bowtie \bowtie \gamma_{j_{p}}$,

$$
\left(\gamma_{i_{1}, \ldots, i_{k}} \bowtie \gamma_{j_{1}, \ldots, j_{p}}\right)_{a d}^{m l}=\delta_{n p}\left\{\theta\left(j_{1}-i_{k}\right) \tau^{b c}+\theta\left(i_{k}-j_{1}\right) \tau^{c b}\right\}\left(\gamma_{i_{1}, \ldots, i_{k}}\right)_{a b}^{m n}\left(\gamma_{j_{1}, \ldots, j_{p}}\right)_{c d}^{p l},
$$

where the arguments of $\gamma_{j}\left(\mathcal{A}_{+}\left(Y_{1}^{j} \mid X^{j}\right), \mathcal{A}_{-}\left(Y_{2}^{j} \mid X^{j}\right)\right)$ are omitted for simplicity. The trace is defined analogously

$$
\operatorname{tr}_{\bowtie}\left(\gamma_{j_{1}, \ldots, j_{p}}\right)=\delta_{m k}\left\{\theta\left(j_{1}-j_{p}\right) \tau^{b a}+\theta\left(j_{p}-j_{1}\right) \tau^{a b}\right\}\left(\gamma_{j_{1}, \ldots, j_{p}}\right)_{a b}^{m k} .
$$


This gives by virtue of (8.28), (8.29)

$$
\begin{aligned}
& \left(g_{j_{1}}^{0} \bowtie \ldots \bowtie g_{j_{n}}^{0}\right)_{a d}^{m l}\left(W_{1}, W_{2} \mid X\right)=\left(\mathfrak{g}^{0}\left(X^{j_{1}} ; \gamma_{j_{1}}\right) \bowtie \ldots \bowtie \mathfrak{g}^{0}\left(X^{j_{n}} ; \gamma_{j_{n}}\right)\right)_{a d}^{m l}\left(W_{1}, W_{2}\right) \\
= & -\left.2^{n-1}\left\{\left(\gamma_{j_{1}}^{+} \bowtie \ldots \bowtie \gamma_{j_{n}}^{+}\right)_{a d}^{m l} \mathfrak{D}_{a d}^{\mathfrak{p}_{m} \mathfrak{p}_{l}}\left(Y_{1}^{j_{1}}, Y_{2}^{j_{n}}, X^{j_{1}}, X^{j_{n}} \mid W_{1}, W_{2}\right) \prod_{k=1}^{n-1} \mathbb{E}_{j_{k}, j_{k+1}}\right\}\right|_{Y=0}
\end{aligned}
$$

and, using again (3.12) and (8.27),

$$
\begin{aligned}
\operatorname{tr}_{\bowtie} & \left(g_{j_{1}}^{0} \bowtie \ldots \bowtie g_{j_{n}}^{0}\right)(X)=\operatorname{tr}_{\bowtie}\left(\mathfrak{g}^{0}\left(X^{j_{1}} ; \gamma_{j_{1}}\right) \bowtie \ldots \bowtie \mathfrak{g}^{0}\left(X^{j_{n}} ; \gamma_{j_{n}}\right)\right) \\
\quad & \left.(-1)^{\mathfrak{p}_{j_{1}}^{1}} 2^{n-1}\left\{\operatorname{tr}_{\bowtie}\left(\gamma_{j_{1}}^{+} \bowtie \ldots \bowtie \gamma_{j_{n}}^{+}\right) \mathbb{E}_{j_{n}, j_{1}} \prod_{k=1}^{n-1} \mathbb{E}_{j_{k}, j_{k+1}}\right\}\right|_{Y=0},
\end{aligned}
$$

where, according to (8.8), (8.9) and (8.10),

$$
\begin{gathered}
\mathbb{E}_{j, k}=\delta^{\mathfrak{p}_{j}^{2} \mathfrak{p}_{k}^{1}} \frac{\mathcal{E}_{j, k}^{\mathfrak{p}_{j}^{2}}}{\mathrm{D}^{j, k}} \\
\mathrm{D}^{j, k}=(4 \pi)^{\frac{M}{2}} \exp \left(\operatorname{sign}(k-j) \frac{i \pi I_{X^{k}-X^{j}}}{4}\right) \sqrt{\left|\operatorname{det}\left(X^{k}-X^{j}\right)\right|} \\
\mathcal{E}_{j, k}^{0}=\exp \left(-\frac{i}{4}\left(X^{k}-X^{j}\right)_{A B}^{-1}\left(Y_{2}^{j} Y_{2}^{j}+Y_{1}^{k} Y_{1}^{k}\right)^{A B}\right) \cos \left(\frac{1}{2}\left(X^{k}-X^{j}\right)_{A B}^{-1} Y_{2}^{j A} Y_{1}^{k B}\right), \\
\mathcal{E}_{j, k}^{1}=i \exp \left(-\frac{i}{4}\left(X^{k}-X^{j}\right)_{A B}^{-1}\left(Y_{2}^{j} Y_{2}^{j}+Y_{1}^{k} Y_{1}^{k}\right)^{A B}\right) \sin \left(\frac{1}{2}\left(X^{k}-X^{j}\right)_{A B}^{-1} Y_{2}^{j A} Y_{1}^{k B}\right) .
\end{gathered}
$$

\section{$8.2 \quad F$-currents}

$F$-currents $\mathcal{J}_{\eta}(Y \mid X)$ are represented by $\mathrm{J}_{\gamma}(Y \mid X)$ at $\gamma_{a b}=\eta \forall a, b$. Space-time $F$-currents are $\mathcal{J}_{\eta}(Y \mid X)$ evaluated at $Y=0$. As explained in Section 4 , the $\rho$-odd part $\eta^{-}$(4.25) does not contribute to $\mathcal{J}_{\eta}(0,0 \mid X)$ (4.26). Hence, space-time $F$-currents are represented by $\mathcal{I}_{g}^{2}$ (6.1) with $g=g_{\left\{0, X ; \eta^{+}\right\}}$.

Naively, this conclusion disagrees with the results of Section 5, where it was shown that, for $M=2$, nonzero contribution to the charges gives $\eta^{-}$(4.25), obeying $\rho\left(\eta^{-}\right)=-\eta^{-}$. However, one should take into account that the $d X$-dependent part of the closed form (5.2) contains derivatives $\frac{\partial}{\partial U^{A}}$ equivalent to $\mathcal{B}_{A}$. Hence, the parameters of charges and currents differ by $M$ factors of $\mathcal{B}_{A}$. As a result, their $\rho$-parities differ by a factor of $(-1)^{\frac{M}{2}}$, which is -1 at $M=2$. Note that the symmetry properties of parameters match for all $M$. However, in the general case, the correspondence is less trivial because, as demonstrated in [28], physical parameters contribute to the closed form (5.2) with additional singular factors that support integration over spinor variables and compensate the mismatch in $\rho$-parity. For example, in the $4 d$ case of $M=4$, the symmetry parameter contributes to the physical charge with the singular factor bilinear in twistor variables, which just brings in a factor of -1 . In the twistor sector, necessary sign factors result from the appropriate choice of integration contours in the twistor space. 
Operator product of $n$ free conformal $F$ - multilinear currents $\mathcal{J}_{\eta_{j}}^{2 k_{j}}$ with $\rho$ - invariant parameters $\eta_{j}$ is given by

$$
\mathcal{J}_{\eta_{1}}^{2 k_{1}}\left(X^{1}\right) \ldots \mathcal{J}_{\eta_{n}}^{2 k_{n}}\left(X^{n}\right)=\left.\left(\frac{\partial^{k_{1}}}{\left(\partial \mu^{1}\right)^{k_{1}}} \cdots \frac{\partial^{k_{n}}}{\left(\partial \mu^{n}\right)^{k_{n}}} E\left(\mathfrak{V}^{0}(\mu)\right)\right)\right|_{\mu=0}
$$

with

$$
\begin{gathered}
\mathfrak{V}^{0}(\mu)=\sum_{j=1}^{\infty} \mu^{j} g_{j}^{0}, \quad g_{j}^{0}=\mathfrak{g}^{0}\left(X^{j} ; \eta_{j}\right) \\
\mathfrak{g}_{a b}^{0 m l}\left(X ; \eta \mid W_{1}, W_{2}\right)=-\left.\eta^{m l}\left(\mathcal{A}_{+}\left(Y_{1} \mid X\right), \mathcal{A}_{-}\left(Y_{2} \mid X\right)\right) \mathfrak{D}_{a b}^{\mathfrak{p}_{m} \mathfrak{p}_{l}}\left(Y_{1}, Y_{2}, X \mid W_{1}, W_{2}\right)\right|_{Y=0} .
\end{gathered}
$$

The formula to be used below for the derivation of OPE and correlators of bilinear $F$-currents is obtained at $k_{1}=\ldots=k_{n}=1$

$$
\mathcal{J}_{\eta_{1}}\left(X^{1}\right) \ldots \mathcal{J}_{\eta_{n}}\left(X^{n}\right)=\left.\left(\frac{\partial}{\partial \mu^{1}} \ldots \frac{\partial}{\partial \mu^{n}} E\left(\mathfrak{V}^{0}(\mu)\right)\right)\right|_{\mu=0}
$$

For $F$-currents, butterfly product (8.32) amounts to the matrix product $\eta_{j} \cdot \eta_{k}$ with respect to color indices, i.e.,

$$
\left(\eta_{i} \cdot \eta_{j}\right)^{m l}=\delta_{n k}\left(\eta_{i}\right)^{m n}\left(\eta_{j}\right)^{k l}, \quad \operatorname{tr} .\left(\eta_{j_{1}} \cdot \eta_{j_{2}}\right)=\delta_{m k}\left(\eta_{j_{1}} \cdot \eta_{j_{2}}\right)^{m k}
$$

Assuming that $\eta_{j}=\eta_{j}{ }^{m_{j} n_{j}}$ has definite parities $\mathfrak{p}_{j}^{1}$ and $\mathfrak{p}_{j}^{2}$ (8.31) with respect to the first and second arguments and that $j_{k} \neq j_{m}$ for $k \neq m$, this gives

$$
\begin{aligned}
& \left(\mathfrak{g}^{0}\left(X^{j_{1}} ; \eta_{j_{1}}\right) \bowtie \ldots \bowtie \mathfrak{g}^{0}\left(X^{j_{n}} ; \eta_{j_{n}}\right)\right)_{a d}^{m l}\left(W_{1}, W_{2}\right) \\
& =-\left.2^{n-1}\left\{\left(\eta_{j_{1}} \cdot \ldots \cdot \eta_{j_{n}}\right)^{m l} \mathfrak{D}_{a d}^{\mathfrak{p}_{m} \mathfrak{p}_{l}}\left(Y_{1}^{j_{1}}, Y_{2}^{j_{n}}, X^{j_{1}}, X^{j_{n}} \mid W_{1}, W_{2}\right) \prod_{k=1}^{n-1} \mathbb{E}_{j_{k}, j_{k+1}}\right\}\right|_{Y=0}, \\
& \operatorname{tr}_{\bowtie}\left(g_{j_{1}}^{0} \bowtie \ldots \bowtie g_{j_{n}}^{0}\right)=\operatorname{tr}_{\bowtie}\left(\mathfrak{g}^{0}\left(X^{j_{1}} ; \eta_{j_{1}}\right) \bowtie \ldots \bowtie \mathfrak{g}^{0}\left(X^{j_{n}} ; \eta_{j_{n}}\right)\right) \\
& =\left.(-1)^{\mathfrak{p}_{j_{1}}^{1}} 2^{n-1}\left\{\operatorname{tr} .\left(\eta_{j_{1}} \cdot \ldots \cdot \eta_{j_{n}}\right) \mathbb{E}_{j_{n}, j_{1}} \prod_{k=1}^{n-1} \mathbb{E}_{j_{k}, j_{k+1}}\right\}\right|_{Y=0} \text {. }
\end{aligned}
$$

These formulae make the computation of OPE and correlators of conserved currents straightforward.

\section{Correlators of free conformal currents}

\subsection{Any $M$ and $3 d$}

From (8.44) it follows that the correlator of $n$ free conformal $F$ - currents $\mathcal{J}_{\eta_{j}}$ with $\rho$ - invariant parameters $\eta_{j}$ can be represented in terms of butterfly algebra $A_{\bowtie}$ as

$$
\left\langle\mathcal{J}_{\eta_{1}}\left(X^{1}\right) \ldots \mathcal{J}_{\eta_{n}}\left(X^{n}\right)\right\rangle=\sum_{\mathcal{S}_{n}} \sum_{r} \frac{1}{r !} \sum_{\substack{k_{1}, \ldots, k_{r} \geq 2 \\ k_{1}+\ldots+k_{r}=n}} \frac{1}{k_{1} \cdots k_{r}} \operatorname{tr}_{\bowtie}\left(g_{j_{1}^{1}, \ldots, j_{k_{1}}^{1}}^{0}\right) \ldots \operatorname{tr} r_{\bowtie}\left(g_{j_{1}^{r}, \ldots, j_{k_{r}}^{r}}^{0}\right),
$$


where $g_{j_{1}, \ldots, j_{k}}^{0}=g_{j_{1}}^{0} \bowtie \ldots \bowtie g_{j_{k}}^{0}$ with $g_{j}^{0}=\mathfrak{g}^{0}\left(X^{j} ; \eta_{j}\right)$ (8.43), and summation over $\mathcal{S}_{n}$ implies symmetrization over indices $j_{1}^{1}, \ldots, j_{k_{1}}^{1}, \ldots, j_{1}^{r}, \ldots, j_{k_{r}}^{r}$. (Note that the terms where at least one $k_{i}=1$ do not contribute because $\operatorname{tr}_{\bowtie}\left(g_{j_{k}}^{0}\right)=0$ in accordance with the fact that, being normally ordered, fundamental currents have zero VEVs.) Connected $n$-point functions of free $F$-currents with $\rho$ invariant parameters $\eta_{j}$ are

$$
\left\langle\mathcal{J}_{\eta_{1}}\left(X^{1}\right) \ldots \mathcal{J}_{\eta_{n}}\left(X^{n}\right)\right\rangle_{\text {con }}=\frac{1}{n} \sum_{\mathcal{S}_{n}} \operatorname{tr}_{\bowtie}\left(g_{1}^{0} \bowtie \ldots \bowtie g_{n}^{0}\right),
$$

where summation is over all permutations of $(1, \ldots, n)$.

Substitution of (8.47) into (9.1), (9.2) gives $n$-point functions of free $F$-currents. These formulae hold for any $M \geq 2$. At $M=2$, they describe $n$-point functions of $3 d$ conformal currents. For any $M$, the primary currents (2.19) are associated with the parameters

$$
\eta_{0}, \quad \eta\left(\partial_{U}\right), \quad \eta\left(\partial_{V}\right), \quad \eta^{A B} \partial_{U^{A}} \partial_{V^{B}}, \quad \text { with } \quad \eta^{A B}=-\eta^{B A} .
$$

\section{$9.24 d$}

As discussed in [4, 5], usual Minkowski space $M^{d}$ is a subspace of the matrix space $\mathcal{M}_{M}$ with appropriate $M$. Embedding of $4 d$ Minkowski space-time into $\mathcal{M}_{4}$ is most conveniently described in terms of two-component indices $\alpha, \beta=1,2$ and $\alpha^{\prime}, \beta^{\prime}=1^{\prime}, 2^{\prime}$ in place of the four-component indices $A, B \ldots$ with the convention that complex conjugation exchanges unprimed and primed indices. We use notation with $A=\left(\alpha, \alpha^{\prime}\right)$ and $Y^{A}=\left(y^{\alpha}, \bar{y}^{\alpha^{\prime}}\right)$, where $\bar{y}^{\alpha^{\prime}}=\overline{y^{\alpha}}$. Two-component indices are raised and lowered according to

$$
A^{\alpha}=\varepsilon^{\alpha \beta} A_{\beta}, \quad A_{\beta}=\varepsilon_{\alpha \beta} A^{\alpha}, \quad \varepsilon_{\alpha \beta}=-\varepsilon_{\beta \alpha}, \quad \varepsilon_{12}=1,
$$

and analogously for primed indices.

In these terms,

$$
X^{A B}=\left(\begin{array}{cc}
X^{\alpha \beta} & X^{\alpha^{\prime} \beta} \\
X^{\alpha \beta^{\prime}} & \bar{X}^{\alpha^{\prime} \beta^{\prime}}
\end{array}\right), \quad Y^{A}=\left(\begin{array}{c}
y^{\alpha} \\
\bar{y}^{\beta^{\prime}}
\end{array}\right)
$$

with $\overline{X^{\alpha \beta^{\prime}}}=X^{\beta \alpha^{\prime}}$ and $\overline{X^{\alpha \beta}}=\bar{X}^{\alpha^{\prime} \beta^{\prime}}$. For Minkowski coordinates, we also use notation $x^{\alpha \beta^{\prime}}$ instead of $X^{\alpha \beta^{\prime}}$. Minkowski time $t$ and space coordinates $x^{i}$ are

$$
X^{\alpha \beta^{\prime}}=t T^{\alpha \beta^{\prime}}+x^{i} \sigma_{i}^{\alpha \beta^{\prime}}, \quad i=1,2,3,
$$

where $T^{\alpha \beta^{\prime}}=\delta^{\alpha \beta^{\prime}}$ while $\sigma_{i}^{\alpha \beta^{\prime}}$ are Hermitian traceless Pauli matrices.

As shown in [5], since $4 d$ Minkowski space is identified with

$$
X^{A B}=\left(\begin{array}{cc}
0 & x^{\alpha^{\prime} \beta} \\
x^{\alpha \beta^{\prime}} & 0
\end{array}\right)
$$

the inertia index of $X^{A B}$ equals to \pm 4 or 0 . As a result, $s(X)=-\operatorname{det}\left(x^{\beta \alpha^{\prime}}\right)$. 
$4 d$ correlators are given by Eqs. (9.1), (8.47) with coordinates (9.6), $Y=(y, \bar{y}), \eta_{j}=$ $\eta_{j}\left(\mathcal{A}_{+}\left(Y_{1}^{j} \mid x^{j}\right), \mathcal{A}_{+}\left(Y_{2}^{j} \mid x^{j}\right)\right), \mathfrak{p}_{j}^{1,2}$ being the parity of $\eta_{j}$ with respect to $Y_{1,2}^{j}$, respectively, and

$$
\begin{gathered}
\mathbb{E}_{j, k}=\delta^{\mathfrak{p}_{j}^{2} \mathfrak{p}_{k}^{1}} \frac{e_{j, k}^{\mathfrak{p}_{j}^{2}}}{(4 \pi)^{2} \operatorname{det}\left(x^{j}-x^{k}\right)}, \\
e_{j, k}^{0}=\exp \left(-\frac{i}{2}\left(x^{k}-x^{j}\right)_{\alpha \beta^{\prime}}^{-1}\left(y_{1}^{k \alpha} \bar{y}_{1}^{k \beta^{\prime}}+y_{2}^{j \alpha} \bar{y}_{2}^{j \beta^{\prime}}\right)\right) \cos \left(\frac{1}{2}\left(x^{k}-x^{j}\right)_{\alpha \beta^{\prime}}^{-1}\left(y_{2}^{j \alpha} \bar{y}_{1}^{k \beta^{\prime}}+y_{1}^{k \alpha} \bar{y}_{2}^{j \beta^{\prime}}\right)\right), \\
e_{j, k}^{1}=i \exp \left(-\frac{i}{2}\left(x^{k}-x^{j}\right)_{\alpha \beta^{\prime}}^{-1}\left(y_{1}^{k \alpha} \bar{y}_{1}^{k \beta^{\prime}}+y_{2}^{j \alpha} \bar{y}_{2}^{j \beta^{\prime}}\right)\right) \sin \left(\frac{1}{2}\left(x^{k}-x^{j}\right)_{\alpha \beta^{\prime}}^{-1}\left(y_{2}^{j \alpha} \bar{y}_{1}^{k \beta^{\prime}}+y_{1}^{k \alpha} \bar{y}_{2}^{j \beta^{\prime}}\right)\right) .
\end{gathered}
$$

Connected $n$-point functions of free $F$-currents with $\rho$-invariant parameters $\eta_{j}$ are

$$
\left\langle\mathcal{J}_{\eta_{1}}\left(x^{1}\right) \ldots \mathcal{J}_{\eta_{n}}\left(x^{n}\right)\right\rangle_{\text {con }}=\frac{1}{n} \sum_{\mathcal{S}_{n}} \operatorname{tr}_{\bowtie}\left(g_{1, \ldots, n}^{0}\right) .
$$

To describe primary currents, that belong to the cohomology group $H^{0}\left(\sigma_{-M n k}^{2}\right)$ of $4 d$ Minkowski current equation where

$$
\sigma_{-M n k}^{2}=\frac{1}{2} d x^{\alpha \beta^{\prime}}\left(\frac{\partial}{\partial v^{\alpha}} \frac{\partial}{\partial \bar{u}^{\beta^{\prime}}}+\frac{\partial}{\partial u^{\alpha}} \frac{\partial}{\partial \bar{v}^{\beta^{\prime}}}\right)=d x^{\alpha \beta^{\prime}}\left(-\frac{\partial}{\partial y_{1}^{\alpha}} \frac{\partial}{\partial \bar{y}_{1}^{\beta^{\prime}}}+\frac{\partial}{\partial y_{2}^{\alpha}} \frac{\partial}{\partial \bar{y}_{2}^{\beta^{\prime}}}\right),
$$

following 442 it is useful to introduce $\mathfrak{s l}_{2}$ generators

$\rho_{+}=y_{1}^{\gamma} \frac{\partial}{\partial y_{2}^{\gamma}}+\bar{y}_{2}^{\gamma^{\prime}} \frac{\partial}{\partial \bar{y}_{1}^{\gamma^{\prime}}}, \quad \rho_{-}=y_{2}^{\gamma} \frac{\partial}{\partial y_{1}^{\gamma}}+\bar{y}_{1}^{\gamma^{\prime}} \frac{\partial}{\partial \bar{y}_{2}^{\gamma^{\prime}}}, \quad \rho_{0}=y_{1}^{\gamma} \frac{\partial}{\partial y_{1}^{\gamma}}+\bar{y}_{2}^{\gamma^{\prime}} \frac{\partial}{\partial \bar{y}_{2}^{\gamma^{\prime}}}-y_{2}^{\gamma} \frac{\partial}{\partial y_{2}^{\gamma}}-\bar{y}_{1}^{\gamma^{\prime}} \frac{\partial}{\partial \bar{y}_{1}^{\gamma^{\prime}}}$,

that commute to $\sigma_{-M n k}^{2}$ and act on the space of bilinear currents and, hence, on the space of parameters.

Treating $\rho_{+}$as a positive grade operator, one can find highest $\mathfrak{s l}_{2}$-vectors in $H^{0}\left(\sigma_{-M n k}^{2}\right)$ to reconstruct full $H^{0}\left(\sigma_{-M n k}^{2}\right)$ by the action of $\rho_{-}$. This gives the following set of parameters

$\eta\left(\frac{\partial}{\partial y_{1}}, \frac{\partial}{\partial y_{2}}\right), \quad \bar{\eta}\left(\frac{\partial}{\partial \bar{y}_{1}}, \frac{\partial}{\partial \bar{y}_{2}}\right), \quad \rho_{-}^{k}\left(\tilde{\eta}\left(\frac{\partial}{\partial y_{2}}, \frac{\partial}{\partial \bar{y}_{1}}\right)\right) \quad$ with $\rho_{-}(\eta)=\left[\eta, \rho_{-}\right] \quad(k=0,1, \ldots)$,

representing all primary currents. Note that here $\tilde{\eta} \rho_{-}$is replaced by $\left[\tilde{\eta}, \rho_{-}\right]$using that this does not affect the final result since $Y=0$ in (8.47).

For example, consider

$$
\tilde{\eta}^{(p, q)}\left(\frac{\partial}{\partial y_{2}}, \frac{\partial}{\partial \bar{y}_{1}}\right)=\eta^{\gamma(p) ; \gamma^{\prime}(q)} \frac{\partial}{\partial y_{2}^{\gamma_{1}}} \ldots \frac{\partial}{\partial y_{2}^{\gamma_{p}}} \frac{\partial}{\partial \bar{y}_{1}^{\gamma_{1}}} \ldots \frac{\partial}{\partial \bar{y}_{1}^{\gamma^{\prime}{ }_{p}}}
$$

Let

$$
\mathfrak{h}_{1}=\frac{1}{2}\left(y_{1}^{\gamma} \frac{\partial}{\partial y_{1}^{\gamma}}-\bar{y}_{1}^{\gamma^{\prime}} \frac{\partial}{\partial \bar{y}_{1}^{\gamma^{\prime}}}\right), \quad \mathfrak{h}_{2}=\frac{1}{2}\left(y_{2}^{\gamma} \frac{\partial}{\partial y_{2}^{\gamma}}-\bar{y}_{2}^{\gamma^{\prime}} \frac{\partial}{\partial \bar{y}_{2}^{\gamma^{\prime}}}\right)
$$


be rank-1 helicity operators in $4 d$ Minkowski space. Evidently,

$$
\mathfrak{h}_{1}(\tilde{\eta})=\frac{1}{2} q \tilde{\eta}, \quad \mathfrak{h}_{2}(\tilde{\eta})=-\frac{1}{2} p \tilde{\eta}
$$

Let $\tilde{\eta}_{k}^{(p, q)}=\frac{1}{k !} \rho^{k}\left(\tilde{\eta}^{(p, q)}\right)$. Clearly, $\tilde{\eta}_{k}^{(p, q)}=0$ for $k>(p+q)$. Since $\mathfrak{h}_{1}\left(\rho_{-}\right)=-\frac{1}{2} \rho_{-}$, $\mathfrak{h}_{2}\left(\rho_{-}\right)=\frac{1}{2} \rho_{-}$,

$$
\mathfrak{h}_{1}\left(\tilde{\eta}_{k}^{(p, q)}\right)=\frac{1}{2}(q-k) \tilde{\eta}_{k}^{(p, q)}, \quad \mathfrak{h}_{2}\left(\tilde{\eta}_{k}^{(p, q)}\right)=-\frac{1}{2}(p-k) \tilde{\eta}_{k}^{(p, q)} .
$$

In particular, for $q=p=2$, this gives

$$
\begin{aligned}
& \tilde{\eta}_{0}^{(2,2)}=\eta^{\alpha \beta ; \alpha^{\prime} \beta^{\prime}} \frac{\partial}{\partial y_{2}^{\alpha}} \frac{\partial}{\partial y_{2}^{\beta}} \frac{\partial}{\partial \bar{y}_{1}^{\alpha^{\prime}}} \frac{\partial}{\partial \bar{y}_{1}^{\beta^{\prime}}}, \\
& \tilde{\eta}_{1}^{(2,2)}=\eta^{\alpha \beta ; \alpha^{\prime} \beta^{\prime}}\left\{\frac{\partial}{\partial y_{1}^{\alpha}} \frac{\partial}{\partial y_{2}^{\beta}} \frac{\partial}{\partial \bar{y}_{1}^{\alpha^{\prime}}} \frac{\partial}{\partial \bar{y}_{1}^{\beta^{\prime}}}+\frac{\partial}{\partial y_{2}^{\alpha}} \frac{\partial}{\partial y_{2}^{\beta}} \frac{\partial}{\partial \bar{y}_{2}^{\alpha^{\prime}}} \frac{\partial}{\partial \bar{y}_{1}^{\beta^{\prime}}}\right\}, \\
& \tilde{\eta}_{2}^{(2,2)}=\eta^{\alpha \beta ; \alpha^{\prime} \beta^{\prime}}\left\{\frac{\partial}{\partial y_{1}^{\alpha}} \frac{\partial}{\partial y_{1}^{\beta}} \frac{\partial}{\partial \bar{y}_{1}^{\alpha^{\prime}}} \frac{\partial}{\partial \bar{y}_{1}^{\beta^{\prime}}}+2 \frac{\partial}{\partial y_{1}^{\alpha}} \frac{\partial}{\partial y_{2}^{\beta}} \frac{\partial}{\partial \bar{y}_{1}^{\alpha^{\prime}}} \frac{\partial}{\partial \bar{y}_{2}^{\beta^{\prime}}}+\frac{\partial}{\partial y_{2}^{\alpha}} \frac{\partial}{\partial y_{2}^{\beta}} \frac{\partial}{\partial \bar{y}_{2}^{\alpha^{\prime}}} \frac{\partial}{\partial \bar{y}_{2}^{\beta^{\prime}}}\right\}, \\
& \tilde{\eta}_{3}^{(2,2)}=\eta^{\alpha \beta ; \alpha^{\prime} \beta^{\prime}}\left\{\frac{\partial}{\partial y_{1}^{\alpha}} \frac{\partial}{\partial y_{1}^{\beta}} \frac{\partial}{\partial \bar{y}_{1}^{\alpha^{\prime}}} \frac{\partial}{\partial \bar{y}_{2}^{\beta^{\prime}}}+\frac{\partial}{\partial y_{1}^{\alpha}} \frac{\partial}{\partial y_{2}^{\beta}} \frac{\partial}{\partial \bar{y}_{2}^{\alpha^{\prime}}} \frac{\partial}{\partial \bar{y}_{2}^{\beta^{\prime}}}\right\}, \\
& \tilde{\eta}_{4}^{(2,2)}=\eta^{\alpha \beta ; \alpha^{\prime} \beta^{\prime}} \frac{\partial}{\partial y_{1}^{\alpha} \frac{\partial}{\partial y_{1}^{\beta}} \frac{\partial}{\partial \bar{y}_{2}^{\alpha^{\prime}}} \frac{\partial}{\partial \bar{y}_{2}^{\beta^{\prime}}} .}
\end{aligned}
$$

The pairs $\tilde{\eta}_{0}^{(2,2)}, \tilde{\eta}_{4}^{(2,2)}$ and $\tilde{\eta}_{1}^{(2,2)}, \tilde{\eta}_{3}^{(2,2)}$ generate the same currents modulo exchange of the fundamental fields $C_{1}$ and $C_{2}$. Indeed, in this case $\tilde{\eta}_{0}^{(2,2)}=\overline{\tilde{\eta}_{4}^{(2,2)}}, \tilde{\eta}_{1}^{(2,2)}=\overline{\tilde{\eta}_{3}^{(2,2)}}, \tilde{\eta}_{2}^{(2,2)}=\overline{\tilde{\eta}_{2}^{(2,2)}}$. As a result, we are left with three independent structures associated with $\tilde{\eta}_{0}^{(2,2)}, \tilde{\eta}_{1}^{(2,2)}$ and $\tilde{\eta}_{2}^{(2,2)}$. These correspond to three types of stress tensors

$$
\begin{aligned}
T^{(1,1)} & =\left.\left(\tilde{\eta}_{0}^{(2,2)} C_{1}\left(y_{1}, \bar{y}_{1}\right) C_{2}\left(y_{2}, \bar{y}_{2}\right)+\tilde{\eta}_{4}^{(2,2)} C_{1}\left(y_{1}, \bar{y}_{1}\right) C_{2}\left(y_{2}, \bar{y}_{2}\right)\right)\right|_{y=\bar{y}=0}, \\
T^{\left(\frac{1}{2}, \frac{1}{2}\right)} & =\left.\left(\tilde{\eta}_{1}^{(2,2)} C_{1}\left(y_{1}, \bar{y}_{1}\right) C_{2}\left(y_{2}, \bar{y}_{2}\right)+\tilde{\eta}_{3}^{(2,2)} C_{1}\left(y_{1}, \bar{y}_{1}\right) C_{2}\left(y_{2}, \bar{y}_{2}\right)\right)\right|_{y=\bar{y}=0} \\
T^{(0,0)} & =\left.\left(\tilde{\eta}_{2}^{(2,2)} C_{1}\left(y_{1}, \bar{y}_{1}\right) C_{2}\left(y_{2}, \bar{y}_{2}\right)\right)\right|_{y=\bar{y}=0},
\end{aligned}
$$

constructed, respectively, from free fields of spins $1, \frac{1}{2}$ and 0 . The respective three-point functions reproduce three known three-point functions of $4 d$ stress tensors (see, e.g., [12, 13]).

Analogously, our construction reproduces $s+1$ independent structures for three-point functions of spin $s$ conserved currents associated with free fields of spins $0,1 / 2, \ldots s / 2$.

\section{Examples}

In this section, obtained results are illustrated by the derivation of $n$-point functions in the generalized space-times with arbitrary $M$. At $M=2$, the resulting formulae give correlators of usual $3 d$ conformal currents. 


\section{$10.1 \quad$ Integer spins}

Consider bosonic $F$-currents. Let

$$
\eta_{j}^{m n}\left(\mathcal{A}_{+}\left(Y_{1} \mid X\right), \mathcal{A}_{-}\left(Y_{2} \mid X\right)\right)=E^{m n} \eta_{j}\left(\mathcal{A}_{+}\left(Y_{1} \mid X\right), \mathcal{A}_{-}\left(Y_{2} \mid X\right)\right)+i \widetilde{E}^{m n} \widetilde{\eta}_{j}\left(\mathcal{A}_{+}\left(Y_{1} \mid X\right), \mathcal{A}_{-}\left(Y_{2} \mid X\right)\right)
$$

with

$$
E^{k m}=\delta^{m k}, \quad \widetilde{E}^{k m}=-\widetilde{E}^{m k}, \quad \widetilde{E}^{m k} \widetilde{E}_{k}^{n}=-\delta^{m n}, \quad \delta_{m}^{m}=\mathcal{N}
$$

(indices are raised and lowered by the $O(\mathcal{N})$ invariant metric $\delta_{n m}$ ), and $\eta_{j}, \widetilde{\eta}_{j}$ obeying

$$
\begin{gathered}
\eta_{j}\left(\mathcal{A}_{+}\left(Y_{1} \mid X\right), \mathcal{A}_{-}\left(Y_{2} \mid X\right)\right)=\eta_{j}\left(\mathcal{A}_{+}\left(i Y_{2} \mid X\right), \mathcal{A}_{-}\left(i Y_{1} \mid X\right)\right), \\
\widetilde{\eta}_{j}\left(\mathcal{A}_{+}\left(Y_{1} \mid X\right), \mathcal{A}_{-}\left(Y_{2} \mid X\right)\right)=-\widetilde{\eta}_{j}\left(\mathcal{A}_{+}\left(i Y_{2} \mid X\right), \mathcal{A}_{-}\left(i Y_{1} \mid X\right)\right)
\end{gathered}
$$

so that, for boson currents, $\eta_{j}$ and $\widetilde{\eta}_{j}$ (10.1) are $\rho$-invariant. Note that, by (10.3), parameters

$\left(\widetilde{\eta}_{j}\right) \eta_{j}$ are (anti)symmetric in color indices, being associated with currents of (odd)even spins.

Substitution of (10.1) into (8.47) gives connected $n$-point functions (9.2)

$\left\langle\mathcal{J}_{\eta_{1}}\left(X^{1}\right) \ldots \mathcal{J}_{\eta_{n}}\left(X^{n}\right)\right\rangle_{c o n}^{b}=\left.2^{n-1} \eta_{(n)}(\mathcal{A}) \sum_{\mathcal{S}_{n}} \frac{\left(\exp i R_{(n)} \cos K_{1,2} \cdots \cos K_{n-1, n} \cos K_{n, 1}\right)(Y)}{\mathrm{D}^{1,2} \ldots \mathrm{D}^{n-1, n} \mathrm{D}^{n, 1}}\right|_{Y=0}$

for boson-boson $F$-currents and

$\left\langle\mathcal{J}_{\eta_{1}}\left(X^{1}\right) \ldots \mathcal{J}_{\eta_{n}}\left(X^{n}\right)\right\rangle_{\text {con }}^{f}=-\left.i^{n} 2^{n-1} \eta_{(n)}(\mathcal{A}) \sum_{\mathcal{S}_{n}} \frac{\left(\exp i R_{(n)} \sin K_{1,2} \cdots \sin K_{n-1, n} \sin K_{n, 1}\right)(Y)}{\mathrm{D}^{1,2} \ldots \mathrm{D}^{n-1, n} \mathrm{D}^{n, 1}}\right|_{Y=0}$

for fermion-fermion $F$-currents. Here

$$
\begin{gathered}
K_{i, j}=\frac{1}{2}\left(X^{j}-X^{i}\right)_{A B}^{-1} Y_{1}^{j A} Y_{2}^{i B} \\
R_{i, j}=\frac{1}{4}\left(X^{i}-X^{j}\right)_{A B}^{-1}\left(Y_{2}^{i A} Y_{2}^{i B}+Y_{1}^{j A} Y_{1}^{j B}\right), \quad R_{(n)}=R_{1,2}+\ldots+R_{n-1, n}+R_{n, 1}
\end{gathered}
$$

and

$$
\begin{gathered}
\eta_{(n)}(\mathcal{A})=\frac{1}{n} \operatorname{tr} .\left(\left(E \eta_{j_{1}}+i \widetilde{E} \widetilde{\eta}_{j_{1}}\right) \cdot \ldots \cdot\left(E \eta_{j_{n}}+i \widetilde{E} \widetilde{\eta}_{j_{n}}\right)\right)(\mathcal{A}) \\
=\frac{\mathcal{N}}{2 n}\left(\prod_{j=1}^{n}\left(\eta_{j}\left(a_{1}^{j}, a_{2}^{j}\right)+\widetilde{\eta}_{j}\left(a_{1}^{j}, a_{2}^{j}\right)\right)+\prod_{j=1}^{n}\left(\eta_{j}\left(a_{1}^{j}, a_{2}^{j}\right)-\widetilde{\eta}_{j}\left(a_{1}^{j}, a_{2}^{j}\right)\right)\right)
\end{gathered}
$$

with

$$
a_{1}^{j}=\mathcal{A}_{+}\left(Y_{1}^{j} \mid X^{j}\right), \quad a_{2}^{j}=\mathcal{A}_{-}\left(Y_{2}^{j} \mid X^{j}\right) .
$$


Note that (10.8) is a consequence of

$$
\operatorname{tr} .(\underbrace{\widetilde{E} \cdot \ldots \cdot \widetilde{E}}_{2 m})=(-1)^{m} \mathcal{N}, \quad \operatorname{tr} .(\underbrace{\widetilde{E} \cdot \ldots \cdot \widetilde{E}}_{2 m+1})=0 .
$$

From more general perspective, Eq. (10.8) expresses $\rho$-invariance of correlators.

Substitution of (10.4) and (10.5) into (9.1) gives $n$-point functions for $F$-currents of integer spins including all disconnected contributions. Note that formulae (10.4) and (10.5) are already projected to parameters $\eta_{j}, \tilde{\eta}_{j}$ (10.1) of definite parities with respect to the first and second arguments. This implies that the condition that parameters $\eta$ should have definite parities can be discarded. We will use this property in the sequel in practical applications of (10.4) and (10.5), only requiring $\eta_{j}, \tilde{\eta}_{j}$ to be $\rho$-invariant, i.e., to obey (10.3).

\subsection{Half-integer spins}

Consider $F$-supercurrents. To distinguish between boson and fermion color indices we denote them by Latin and Greek letters, respectively (the latter should not be confused with spinor indices). Hence, a $F$-supercurrent acquires the form

$$
\mathcal{J}_{\eta}\left(Y_{1}, Y_{2} \mid X\right)=\frac{1}{2}\left(\eta^{m \nu} \hat{\mathcal{T}}_{m \nu}\left(Y_{1}, Y_{2} \mid X\right)+\eta^{\nu m} \hat{\mathcal{T}}_{\nu m}\left(Y_{1}, Y_{2} \mid X\right)\right)
$$

where parameters are required to be $\rho$ - invariant, i.e.,

$$
\eta^{m \nu}\left(\mathcal{A}_{+}\left(Y_{1} \mid X\right), \mathcal{A}_{-}\left(Y_{2} \mid X\right)\right)=i \eta^{\nu m}\left(i \mathcal{A}_{-}\left(Y_{2} \mid X\right), i \mathcal{A}_{+}\left(Y_{1} \mid X\right)\right) .
$$

Let

$$
\eta_{j}^{m \nu}\left(\mathcal{A}_{+}\left(Y_{1} \mid X\right), \mathcal{A}_{-}\left(Y_{2} \mid X\right)\right)=E^{m \nu} \eta_{j}\left(\mathcal{A}_{+}\left(Y_{1} \mid X\right), \mathcal{A}_{-}\left(Y_{2} \mid X\right)\right)+i \widetilde{E}^{m \nu} \widetilde{\eta}_{j}\left(\mathcal{A}_{+}\left(Y_{1} \mid X\right), \mathcal{A}_{-}\left(Y_{2} \mid X\right)\right)
$$

with

$$
E^{m \nu}=E^{\nu m}, \quad \widetilde{E}^{m \nu}=-\widetilde{E}^{\nu m}, \quad \widetilde{E}^{\mu k} \widetilde{E}_{k}^{\nu}=-\delta^{\mu \nu}, \quad E^{\mu k} E_{k \mu}=\mathcal{N} .
$$

From (10.11) it follows

$$
\begin{aligned}
& \eta_{j}\left(\mathcal{A}_{+}\left(Y_{1} \mid X\right), \mathcal{A}_{-}\left(Y_{2} \mid X\right)\right)=i \eta_{j}\left(i \mathcal{A}_{-}\left(Y_{2} \mid X\right), i \mathcal{A}_{+}\left(Y_{1} \mid X\right)\right), \\
& \widetilde{\eta}_{j}\left(\mathcal{A}_{+}\left(Y_{1} \mid X\right), \mathcal{A}_{-}\left(Y_{2} \mid X\right)\right)=-i \widetilde{\eta}_{j}\left(i \mathcal{A}_{-}\left(Y_{2} \mid X\right), i \mathcal{A}_{+}\left(Y_{1} \mid X\right)\right) .
\end{aligned}
$$

For supercurrents, 8.47) is different from zero at $n=2 m$. Taking into account that parameters $\eta_{j}$ (4.22) are Grassmann odd, analogously to the case of bosonic currents, the substitution of (10.12) into (8.47) gives by virtue of (8.9), (8.10), (10.11) and (10.13)

$$
\begin{aligned}
& \left\langle\mathcal{J}_{\eta_{1}}\left(X^{1}\right) \ldots \mathcal{J}_{\eta_{2 m}}\left(X^{2 m}\right)\right\rangle_{\text {con }}=i^{m} 2^{-1} \eta_{(2 m)}(\mathcal{A}) \sum_{\mathcal{S}_{2 m}} \frac{(-1)^{\pi_{\mathcal{S}_{2 m}}}}{\mathrm{D}^{1,2} \ldots \mathrm{D}^{2 m-1,2 m} \mathrm{D}^{2 m, 1}} \\
& \left(-\cos K_{1,2} \sin K_{2,3} \ldots \cdots \cos K_{2 p-1,2 p} \sin K_{2 p, 2 p+1} \cdots \sin K_{2 m, 1}\right. \\
& \left.+\sin K_{1,2} \cos K_{2,3} \ldots \sin K_{2 p-1,2 p} \cos K_{2 p, 2 p+1} \cdots \cos K_{2 m, 1}\right)\left.(Y) \exp \left[i R_{(2 m)}(Y)\right]\right|_{Y=0}
\end{aligned}
$$


with $\mathrm{D}^{i j}$ (8.38), $K$ (10.6), $R$ (10.7) and $\eta_{(2 p)}(\mathcal{A})(10.8)\left(\pi_{\mathcal{S}_{2 m}}\right.$ is the parity of a permutation of $\mathcal{S}_{2 m}$ ). Substitution of (10.15) into (9.1) gives $n$-point functions for $F$-supercurrents of half-integer spins, including disconnected contributions.

\subsection{Correlators of primary currents of integer spins}

\subsubsection{General case}

As mentioned in Section 9.1, primary currents in the matrix space are associated with the parameters (9.3). The primaries associated with antisymmetric tensor $\eta^{A B}$ we call special. Since, at $M=2, \eta^{\alpha \beta} \sim \epsilon^{\alpha \beta}$, in that case there is a single spin zero special current that has dimension $\Delta=2$.

In the variables $U, V(2.28)$, the functions $K_{i, j}(10.6)$ and $R_{i, j}$ (10.7) have the form

$$
\begin{aligned}
K_{i, j} & =\frac{1}{2}\left(X^{j}-X^{i}\right)_{A B}^{-1}\left(U^{i A}+V^{i A}\right)\left(V^{j B}-U^{j B}\right), \\
R_{i, j} & =\frac{1}{4}\left(X^{i}-X^{j}\right)_{A B}^{-1}\left(\left(V^{i A}+U^{i A}\right)\left(V^{i B}+U^{i B}\right)+\left(V^{j A}-U^{j A}\right)\left(V^{j B}-U^{j B}\right)\right) .
\end{aligned}
$$

Setting for example in (10.1)

$$
\eta_{j}=\eta_{j}\left(\partial_{U^{j}}\right), \quad \widetilde{\eta}_{j}=\widetilde{\eta}_{j}\left(\partial_{U^{j}}\right),
$$

satisfying (10.3), we obtain that the nonzero contribution to correlators comes from

$$
\begin{aligned}
& P_{i, j}=\left.K_{i, j}\right|_{V=0}=-\frac{1}{2}\left(X^{i}-X^{j}\right)_{A B}^{-1} U^{i A} U^{j B} \\
& Q_{(p)}=\left.R_{(p)}\right|_{V=0}=Q_{1,2,3}+\ldots+Q_{p-2, p-1, p}+Q_{p-1, p, 1}+Q_{p, 1,2},
\end{aligned}
$$

where

$$
Q_{i, j, k}=\frac{1}{4}\left(\left(X^{i}-X^{j}\right)_{A B}^{-1}+\left(X^{j}-X^{k}\right)_{A B}^{-1}\right) U^{j A} U^{j B} .
$$

Substitution of (10.18) into (10.4), (10.5) gives

$$
\begin{gathered}
\left\langle\mathcal{J}_{\eta_{1}}\left(X^{1}\right) \ldots \mathcal{J}_{\eta_{n}}\left(X^{n}\right)\right\rangle_{c o n}^{b}=\left.2^{n-1} \eta_{(n)}\left(\partial_{U}\right) \sum_{\mathcal{S}_{n}} \frac{\left(\cos Q_{(n)} \cos P_{1,2} \cdots \cos P_{n-1, n} \cos P_{n, 1}\right)(U)}{\mathrm{D}^{1,2} \ldots \mathrm{D}^{n-1, n} \mathrm{D}^{n, 1}}\right|_{U=0}, \\
\left\langle\mathcal{J}_{\eta_{1}}\left(X^{1}\right) \ldots \mathcal{J}_{\eta_{2 m}}\left(X^{2 m}\right)\right\rangle_{c o n}^{f}=\left.(-1)^{m+1} 2^{2 m-1} \eta_{(2 m)}\left(\partial_{U}\right) \sum_{\mathcal{S}_{2 m}} \frac{\left(\cos Q_{(2 m)} \sin P_{1,2} \cdots \sin P_{2 m, 1}\right)(U)}{\mathrm{D}^{1,2} \ldots \mathrm{D}^{2 m-1,2 m} \mathrm{D}^{2 m, 1}}\right|_{U=0}, \\
\left\langle\mathcal{J}_{\eta_{1}}\left(X^{1}\right) \ldots \mathcal{J}_{\eta_{2 m+1}}\left(X^{2 m+1}\right)\right\rangle_{c o n}^{f}= \\
\left.(-1)^{m} 2^{2 m} \eta_{(2 m+1)}\left(\partial_{U}\right) \sum_{\mathcal{S}_{2 m+1}} \frac{\left(\sin Q_{(2 m+1)} \sin P_{1,2} \cdots \sin P_{2 m, 2 m+1} \sin P_{2 m+1,1}\right)(U)}{\mathrm{D}^{1,2} \ldots \mathrm{D}^{2 m, 2 m+1} \mathrm{D}^{2 m+1,1}}\right|_{U=0}
\end{gathered}
$$


(Recall that $\eta_{(n)}(\mathcal{A})$ is given in (10.8).) In the derivation of (10.22)-(10.24) one should take into account antisymmetry of $\left.R_{i, j}\right|_{V=0}$ and $\left.K_{i, j}\right|_{V=0}$ in $i, j$ and full symmetrization of the r.h.s. 's of (10.4) and (10.5), which effectively produces the factors of $\cos Q_{\ldots}$ or $\sin Q_{\ldots}$ via appropriate (anti)symmetrization of $\exp i R_{(n)}$ in Eqs. (10.4) and (10.5).

As anticipated, Eqs. (10.22)-(10.24) are in accordance with the results of [9, 15].

\subsubsection{Two-point function and unitarity}

For primary currents with parameters $\eta_{j}^{m n}$ of the form (10.1) with $\eta_{j}$ and $\widetilde{\eta}_{j}$ (10.18), taking into account that $Q_{(2)}=0$, we obtain from $(\overline{10.22})$ and $(\sqrt[10.23)]{)}$ in the boson-boson and fermionfermion cases, respectively,

$$
\begin{aligned}
& \left\langle\mathcal{J}_{\eta_{1}}\left(X^{1}\right) \mathcal{J}_{\eta_{2}}\left(X^{2}\right)\right\rangle^{b}=-\left.\frac{2 \mathcal{N} \exp \left(\frac{i \pi I_{X^{1}-X^{2}}}{2}\right)}{(4 \pi)^{M}\left|\operatorname{det}\left(X^{2}-X^{1}\right)\right|}\left(\eta_{1} \eta_{2}+\widetilde{\eta}_{1} \widetilde{\eta}_{2}\right)\left(\partial_{U^{1}}, \partial_{U^{2}}\right) \cos ^{2}\left(P\left(U^{1}, U^{2}\right)\right)\right|_{U^{1}=U^{2}=0}, \\
& \left\langle\mathcal{J}_{\eta_{1}}\left(X^{1}\right) \mathcal{J}_{\eta_{2}}\left(X^{2}\right)\right\rangle^{f}=\left.\frac{2 \mathcal{N} \exp \left(\frac{i \pi I_{X^{1}-X^{2}}}{2}\right)}{(4 \pi)^{M}\left|\operatorname{det}\left(X^{2}-X^{1}\right)\right|}\left(\eta_{1} \eta_{2}+\widetilde{\eta}_{1} \widetilde{\eta}_{2}\right)\left(\partial_{U^{1}}, \partial_{U^{2}}\right) \sin ^{2}\left(P\left(U^{1}, U^{2}\right)\right)\right|_{U^{1}=U^{2}=0},
\end{aligned}
$$

where

$$
P\left(U^{1}, U^{2}\right)=-\frac{1}{2}\left(X^{1}-X^{2}\right)_{A B}^{-1} U^{1 A} U^{2 B}
$$

Since at $M=2, I_{X^{1}-X^{2}}=\{-2,0,2\}$, as anticipated,

$$
\left\langle\mathcal{J}_{\eta_{1}}\left(X^{1}\right) \mathcal{J}_{\eta_{2}}\left(X^{2}\right)\right\rangle=\left\langle\mathcal{J}_{\eta_{2}}\left(X^{2}\right) \mathcal{J}_{\eta_{1}}\left(X^{1}\right)\right\rangle \quad \forall X^{1}, X^{2} ; \quad M=2 .
$$

Since the overall sign in front of the two-point function is different for $I_{X^{1}-X^{2}}=0$ and $I_{X^{1}-X^{2}} \neq$ 0 , for $M=2$ Eqs. (10.25), (10.26) can be rewritten in the form

$$
\begin{gathered}
\left\langle\mathcal{J}_{\eta_{1}}\left(X^{1}\right) \mathcal{J}_{\eta_{2}}\left(X^{2}\right)\right\rangle^{b}=-\left.\frac{2 \mathcal{N}(4 \pi)^{-2}}{\operatorname{det}\left(X^{2}-X^{1}\right)}\left(\eta_{1} \eta_{2}+\widetilde{\eta}_{1} \widetilde{\eta}_{2}\right)\left(\partial_{U^{1}}, \partial_{U^{2}}\right) \cos ^{2}\left(P\left(U^{1}, U^{2}\right)\right)\right|_{U^{1}=U^{2}=0}, \\
\left\langle\mathcal{J}_{\eta_{1}}\left(X^{1}\right) \mathcal{J}_{\eta_{2}}\left(X^{2}\right)\right\rangle^{f}=\left.\frac{2 \mathcal{N}(4 \pi)^{-2}}{\operatorname{det}\left(X^{2}-X^{1}\right)}\left(\eta_{1} \eta_{2}+\widetilde{\eta_{1}} \widetilde{\eta}_{2}\right)\left(\partial_{U^{1}}, \partial_{U^{2}}\right) \sin ^{2}\left(P\left(U^{1}, U^{2}\right)\right)\right|_{U^{1}=U^{2}=0}
\end{gathered}
$$

To find correlators of special primaries, we set $\eta_{1}\left(\partial_{Y_{1}^{1}}, \partial_{Y_{2}^{1}}\right)=\eta^{A B} \partial_{U^{A}} \partial_{V^{B}}, \eta_{2}\left(\partial_{Y_{1}^{2}}, \partial_{Y_{2}^{2}}\right)=$ $\eta^{A B} \partial_{U^{\prime} A} \partial_{V^{\prime} B}$ in (10.1) with $\eta^{A B}=-\eta^{B A}, \eta^{A B}=-\eta^{B A}(\tilde{\eta}=0)$. From (10.5), we obtain

$$
\left\langle\mathcal{J}_{\eta}(X) \mathcal{J}_{\eta^{\prime}}\left(X^{\prime}\right)\right\rangle=-\frac{\mathcal{N} \exp \left(\frac{i \pi I_{X-X^{\prime}}}{2}\right)\left(X^{\prime}-X\right)_{A B}^{-1}\left(X^{\prime}-X\right)_{C D}^{-1} \eta^{A C} \eta^{B D}}{(4 \pi)^{M}\left|\operatorname{det}\left(X^{\prime}-X\right)\right|} .
$$

At $M=2$ the special primaries describe $\Delta=2$ scalar currents with $\eta^{\alpha \beta}=\eta^{\alpha \beta}=\epsilon^{\alpha \beta}$. Since $\left(X^{\prime}-X\right)_{\alpha \beta}^{-1}\left(X^{\prime}-X\right)_{\gamma \delta}^{-1} \epsilon^{\alpha \gamma} \epsilon^{\beta \delta}=2 \operatorname{det}^{-1}\left(X^{\prime}-X\right)$, this gives

$$
\left\langle\mathcal{J}_{\eta}(X) \mathcal{J}_{\eta^{\prime}}\left(X^{\prime}\right)\right\rangle=\frac{\mathcal{N}}{8 \pi^{2} \operatorname{det}^{2}\left(X^{\prime}-X\right)} .
$$


As anticipated, the signs of two-point functions respect unitarity. Indeed, suppose that a state

$$
|A\rangle=\int_{\Sigma} \phi^{n m}(X) J_{n m}(X)|0\rangle
$$

is represented by an integral over a space-like surface $\Sigma$ with some measure $\phi^{n m}(X)$. (More precisely, $\Sigma$ is some local Cauchy bundle introduced in [5]). In particular, at $M=2$, for any space-like $\Sigma$,

$$
I_{X-X^{\prime}}=0 \quad \forall X, X^{\prime} \in \Sigma .
$$

In this case, the two-point functions (10.27) and (10.28) turn out to be positive-definite for the currents of even spins and negative-definite for the currents of odd spins. This just matches the property (4.17) that the $\rho$-invariant currents of (odd)even spins are (anti)Hermitian, implying that $\langle A \mid A\rangle>0$ for all spins. (Here it is important that the parameters $\eta_{i}^{n m}$ (10.1) are Hermitian for real $\eta$ and $\widetilde{\eta})$.

\subsubsection{Three-point function}

For primary parameters (10.1) of the form

$$
\eta_{j}^{m n}=\eta_{j}^{m n}\left(\partial_{U^{j}}\right), \quad \widetilde{\eta}_{j}^{m n}=\widetilde{\eta}_{j}^{m n}\left(\partial_{U^{j}}\right),
$$

Eqs. (10.22) and (10.24) give for boson-boson and fermion-fermion currents, respectively,

$$
\begin{aligned}
& \left\langle\mathcal{J}_{\eta_{1}}\left(X^{1}\right) \mathcal{J}_{\eta_{2}}\left(X^{2}\right) \mathcal{J}_{\eta_{3}}\left(X^{3}\right)\right\rangle^{b}=\frac{\mathcal{N}}{\pi^{3 M / 2}} \exp \left(i \frac{\pi}{4}\left(I_{X^{1}-X^{2}}+I_{X^{2}-X^{3}}+I_{X^{1}-X^{3}}\right)\right) \\
& \left|\operatorname{det}\left(X^{1}-X^{2}\right) \operatorname{det}\left(X^{1}-X^{3}\right) \operatorname{det}\left(X^{2}-X^{3}\right)\right|^{-\frac{1}{2}} \\
& \left(\eta_{1}\left(\partial_{U^{1}}\right)\left(\eta_{2}\left(\partial_{U^{2}}\right) \eta_{3}\left(\partial_{U^{3}}\right)+\widetilde{\eta}_{2}\left(\partial_{U^{2}}\right) \widetilde{\eta}_{3}\left(\partial_{U^{3}}\right)\right)+\widetilde{\eta}_{1}\left(\partial_{U^{1}}\right)\left(\widetilde{\eta}_{2}\left(\partial_{U^{2}}\right) \eta_{3}\left(\partial_{U^{3}}\right)+\eta_{2}\left(\partial_{U^{2}}\right) \widetilde{\eta}_{3}\left(\partial_{U^{3}}\right)\right)\right) \\
& \left.\cos \left(Q_{1,2,3}+Q_{2,3,1}+Q_{3,1,2}\right) \cos \left(P_{1,2}\right) \cos \left(P_{2,3}\right) \cos \left(P_{3,1}\right)\left(U^{1}, U^{2}, U^{3}\right)\right|_{U^{1}=U^{2}=U^{3}=0} \\
& \left\langle\mathcal{J}_{\eta_{1}}\left(X^{1}\right) \mathcal{J}_{\eta_{2}}\left(X^{2}\right) \mathcal{J}_{\eta_{3}}\left(X^{3}\right)\right\rangle^{f}=-\frac{\mathcal{N}}{\pi^{3 M / 2}} \exp \left(i \frac{\pi}{4}\left(I_{X^{1}-X^{2}}+I_{X^{2}-X^{3}}+I_{X^{1}-X^{3}}\right)\right) \\
& \left|\operatorname{det}\left(X^{1}-X^{2}\right) \operatorname{det}\left(X^{1}-X^{3}\right) \operatorname{det}\left(X^{2}-X^{3}\right)\right|^{-\frac{1}{2}} \\
& \left(\eta_{1}\left(\partial_{U^{1}}\right)\left(\eta_{2}\left(\partial_{U^{2}}\right) \eta_{3}\left(\partial_{U^{3}}\right)+\widetilde{\eta}_{2}\left(\partial_{U^{2}}\right) \widetilde{\eta}_{3}\left(\partial_{U^{3}}\right)\right)+\widetilde{\eta}_{1}\left(\partial_{U^{1}}\right)\left(\widetilde{\eta}_{2}\left(\partial_{U^{2}}\right) \eta_{3}\left(\partial_{U^{3}}\right)+\eta_{2}\left(\partial_{U^{2}}\right) \widetilde{\eta}_{3}\left(\partial_{U^{3}}\right)\right)\right) \\
& \left.\sin \left(Q_{1,2,3}+Q_{2,3,1}+Q_{3,1,2}\right) \sin \left(P_{1,2}\right) \sin \left(P_{2,3}\right) \sin \left(P_{3,1}\right)\left(U^{1}, U^{2}, U^{3}\right)\right|_{U^{1}=U^{2}=U^{3}=0}
\end{aligned}
$$

\footnotetext{
${ }^{1}$ Integration over a general (i.e., not necessarily space-like) surface will not lead to an integral of some expression of a definite sign since in this case $I_{X-X^{\prime}}$ will take all possible values for various $X, X^{\prime} \in \Sigma$. Nevertheless, the final result should be positive because the space of states resulting from the integration over space-like surfaces, as well as over twistor space, spans the full Hilbert space of states $H$, so that any other integration prescription will result in a vector from $H$.
} 
with

$$
P_{i, j}=-\frac{1}{2}\left(X^{i}-X^{j}\right)_{A B}^{-1} U^{i A} U^{j B}, \quad Q_{i, j, k}=\frac{1}{4}\left(\left(X^{i}-X^{j}\right)_{A B}^{-1}+\left(X^{j}-X^{k}\right)_{A B}^{-1}\right) U^{j A} U^{j B} .
$$

For $M=2$, the factor of $\exp \left(i \frac{\pi}{4}\left(I_{X^{1}-X^{2}}+I_{X^{2}-X^{3}}+I_{X^{1}-X^{3}}\right)\right)$ is sensitive to the order of $\mathcal{J}_{\eta_{1}}\left(X^{1}\right), \mathcal{J}_{\eta_{2}}\left(X^{2}\right), \mathcal{J}_{\eta_{3}}\left(X^{3}\right)$ in the correlator. However, as anticipated, for space-like separation of all three points with $I_{X^{1}-X^{2}}=I_{X^{1}-X^{3}}=I_{X^{2}-X^{3}}=0$ the sign factor is 1 independently of the order of currents.

Consider one special and two regular primary currents setting in $(10.1) \widetilde{\eta}=0, \eta_{1}\left(\partial_{Y_{1,2}^{1}}\right)=$ $\eta_{1}^{A B} \partial_{U^{1 A}} \partial_{V^{1 B}}$ with some antisymmetric $\eta_{1}^{A B}, \eta_{2}\left(\partial_{Y_{1,2}^{2}}\right)=\eta_{2}\left(\partial_{U^{2}}\right)$ and $\eta_{3}\left(\partial_{Y_{1,2}^{3}}\right)=\eta_{3}\left(\partial_{U^{3}}\right)$. From (10.5) it follows

$$
\begin{aligned}
& \left\langle\mathcal{J}_{\eta_{1}}\left(X^{1}\right) \mathcal{J}_{\eta_{2}}\left(X^{2}\right) \mathcal{J}_{\eta_{3}}\left(X^{3}\right)\right\rangle=\frac{-4 i \mathcal{N}}{(4 \pi)^{3 M / 2}} \exp \left(i \frac{\pi}{4}\left(I_{X^{1}-X^{2}}+I_{X^{2}-X^{3}}+I_{X^{1}-X^{3}}\right)\right) \\
& \left(\left|\operatorname{det}\left(X^{1}-X^{2}\right) \operatorname{det}\left(X^{1}-X^{3}\right) \operatorname{det}\left(X^{2}-X^{3}\right)\right|\right)^{-\frac{1}{2}} \eta_{1}^{A B} \eta_{2}\left(\partial_{U^{2}}\right) \eta_{3}\left(\partial_{U^{3}}\right) \\
& \left.\left(X^{1}-X^{2}\right)_{A C}^{-1}\left(X^{3}-X^{1}\right)_{B D}^{-1} U^{2 C} U^{3 D}\left(\cos \left(Q_{2,3,1}+Q_{1,2,3}\right) \sin P_{2,3}\right)\left(U^{2}, U^{3}\right)\right|_{U^{2}=U^{3}=0} .
\end{aligned}
$$

For two special and one regular currents we set in (10.1) $\tilde{\eta}=0, \eta_{j}\left(\partial_{Y_{1,2}^{j}}\right)=\eta_{j}^{A B} \partial_{U^{j} A} \partial_{V^{j} B}$, with some antisymmetric $\eta_{j}^{A B}$ with $j=1,2$ and $\eta_{3}\left(\partial_{Y_{1,2}^{3}}\right)=\eta_{3}\left(\partial_{U^{3}}\right)$. From (10.5) it follows

$$
\begin{aligned}
& \left\langle\mathcal{J}_{\eta_{1}}\left(X^{1}\right) \mathcal{J}_{\eta_{2}}\left(X^{2}\right) \mathcal{J}_{\eta_{3}}\left(X^{3}\right)\right\rangle=\frac{4 \mathcal{N}}{(4 \pi)^{3 M / 2}} \exp \left(i \frac{\pi}{4}\left(I_{X^{1}-X^{2}}+I_{X^{2}-X^{3}}+I_{X^{1}-X^{3}}\right)\right) \\
& \left(\left|\operatorname{det}\left(X^{1}-X^{2}\right) \operatorname{det}\left(X^{1}-X^{3}\right) \operatorname{det}\left(X^{2}-X^{3}\right)\right|\right)^{-\frac{1}{2}} \eta_{1}^{A D} \eta_{2}^{C B} \eta_{3}\left(\partial_{U^{3}}\right) \\
& \left.\left(X^{1}-X^{2}\right)_{A C}^{-1}\left(X^{2}-X^{3}\right)_{B F}^{-1}\left(X^{3}-X^{1}\right)_{E D}^{-1} U^{3 E} U^{3 F} \sin \left(Q_{2,3,1}\right)\left(U^{3}\right)\right|_{U^{3}=0} .
\end{aligned}
$$

\section{Conclusion}

In this paper, operator algebra of $3 d$ conserved currents is reconstructed in terms of certain associative algebra $\mathbf{M}$ of distributions $\mathfrak{g}(Y)$ in the twistor space. Remarkably, description of OPE of the infinite tower of HS currents turns out to be much simpler than for a finite set. The reason is that, as long as distributions $\mathfrak{g}(Y)$ are kept arbitrary, their products at different $Y$ are well defined even at coinciding space-time points $X$. On the other hand, to describe OPE of currents of particular spins, it is necessary to consider appropriate derivatives of $\mathfrak{g}(Y)$ at $Y=0$ leading to (derivatives of) $\delta(Y)$ at $Y=0$ in the operator product. To regularize such expressions, one has to consider currents $J(Y \mid X)$ at different $X$.

Once algebra $\mathbf{M}$ is known, it is easy to reconstruct the dependence on space-time coordinates $X$, which is completely determined by the unfolded equations in terms of $Y$-dependence. Practically, the map is given by the generalized $\mathcal{D}$ function which can be called twistor-toboundary $\mathcal{D}$-function (propagator). This step is insensitive to particular realization of conserved currents since the conservation condition for conformal currents completely determines 
twistor-to-boundary $\mathcal{D}$-function. As a result, space-time operator algebra is determined by the twistor algebra $\mathbf{M}$ which encodes full information on the dynamical origin of the system.

For currents built from free massless fields, after an appropriate half-Fourier transform, algebra $\mathbf{M}$ coincides with the universal enveloping algebra of the conformal HS algebra $[16$. This conclusion fits the analysis of [14, 15] on the bulk side, where the computation performed in terms of HS algebra was essentially based on HS symmetry, allowing the authors of [15] to find all connected $n$-point functions up to overall coefficients. Similarly, the computation of this paper, allowing to determine $n$-point functions with exact relative coefficients, is controlled by the symmetry associated with $\mathbf{M}$. Since $\mathbf{M}$ relates operator products involving different numbers of currents, it was called multiparticle algebra in [16]. (Note that it has the meaning of a multiparticle symmetry from the bulk point of view where boundary currents correspond to elementary fields.) The final result turns out to be remarkably simple both for the operator algebra and for $n$-point functions, being formulated in terms of butterfly product constructed from the star product of the HS algebra. In particular, the generating function of $n$-point functions has the suggestive form of certain determinant with respect to butterfly product.

Obtained results may have different applications. A particularly interesting direction is to analyze deformations of operator algebras to be associated with deformations of the underlying free dynamical system, i.e., interactions. For example, $A d S_{4} / C F T_{3} \mathrm{HS}$ algebras (see [38] and references therein) contain no free continuous parameters. Hence, the corresponding $3 d$ operator algebras are rigid in agreement with the conclusion of Maldacena and Zhiboedov [22]. On the other hand, $A d S_{3} / C F T_{2}$ HS algebras contain a free parameter $\nu$ [41]. Hence, in agreement with the analysis of $H S A d S_{3} / C F T_{2}$ correspondence (see [43] and references therein), the boundary algebras are not unique.

On the other hand, as explained in more detail in [16], $\mathbf{M}$ is a promising candidate for the symmetry underlying a string-like extension of HS theory, which we call multiparticle theory. Hopefully, further study along these lines may shed light on the structure of HS theory with mixed-symmetry fields and, eventually, on yet unknown HS multiparticle version of String Theory. It is tempting to speculate that the origin of butterfly formulae for the operator algebra and $n$-point functions should receive natural explanation in terms of the multiparticle theory.

Amplitudes can be associated with $n$-point functions carrying stripped indices of creation and annihilation operators. Interaction deformation of such $n$-point functions, computed in this paper for free currents, may help to clarify deeper structures underlying tremendous progress in the analysis of multiparticle processes of field theory. Parallelism with sophisticated fieldtheoretic methods due to twistor description is obvious (see, e.g., 44] and references therein).

Wightman functions of currents $\left\langle J\left(X^{1}\right) J\left(X^{2}\right) \ldots J\left(X^{n}\right)\right\rangle$ evaluated in this paper should be distinguished from chronological functions. The latter can be easily obtained from the former at least away from singularities via insertion of appropriate step-functions in time. On the other hand, Wightman functions contain additional factors, that depend on the causal relations between coordinates $X^{i}$ and originate from the accurate definition of the involved $\mathcal{D}$-functions. Both in our scheme and in that of [14, 15] $\mathcal{D}$-functions and Green functions result from Gaussian integrals in the twistor space. In our approach, signs of $\mathcal{D}$-functions are 
determined by evaluation of Gaussian integrals in the complexified Fock-Siegel space [28] which is a twistor extension of the usual Siegel space [29, 30]. It would be interesting to extend this technics to the bulk computations of [14, 15] where evaluation of Gaussians was so far a bit formal, hence leaving undetermined sign factors resulting from the square root of the determinant.

Being based on unfolded dynamics, our construction can be easily extended to a larger space where the symmetry, originally interpreted as conformal at the boundary, acts. This is achieved via extension of the unfolded current conservation equations, that still have the form of flatness conditions (2.50). Most natural option is to go to the bulk $A d S$ space. This is achieved via extension of $\mathcal{D}$-functions to the bulk by solving unfolded equations (2.1) with the initial data (3.5), that gives a twistor-to-bulk extension of the rank-two $\mathcal{D}$-function. In fact, up to details distinguishing between $\mathcal{D}$-functions and Green functions, the respective twistor-tobulk $\mathcal{D}$-functions (propagators) that indeed respect the initial data (3.5) in the twistor space are well known (see, e.g., [11, 15 and references therein). In accordance with the general analysis of [24], this simple observation uplifts the whole setting from the boundary to the bulk in a very straightforward way, making boundary and bulk computations literally equivalent. In particular, our computation reproduces the results of Didenko and Skvortsov [15] on $3 d$ connected $n$-point functions, determining all relative coefficients for different $n$ and extending them to supercurrents. This approach works equally well in higher dimensions allowing us, in particular, to evaluate $n$-point current correlators in four dimensions in Section 9.2.

As argued in [24], equivalence of the boundary and bulk pictures should hold beyond the level of free fields as well. However, apart from two specific HS models corresponding to free boundary theories, explicit relation is more involved requiring solution of nonlinear unfolded equations in the both of dual pictures. To make the $A d S_{4} / C F T_{3}$ correspondence complete at the nonlinear quantum level, one should construct a generating functional invariant under (appropriately deformed) multiparticle symmetries. As shown in [45], in unfolded dynamics approach, gauge invariance of such a functional implies that it is represented by an integral of a closed form, which is independent of local variations of the integration surface. (This is analogous to the charge $Q$ (5.1) that can be equally well evaluated via integration over twistor space or space-time.) Hence, the corresponding generating functional can be evaluated via integration over $(i)$ twistor space, $(i i)$ partially twistor space and partially boundary surface, or (iii) over bulk, giving the same result. Such an equivalence (duality) may seem obscure unless manifest equivalence of different formulations via unfolded dynamics is accounted.

\section{Acknowledgments}

The authors are grateful to V. Didenko, E. Feigin, E. Skvortsov, I.Tipunin, I. Tyutin and, especially, M. Soloviev and B. Voronov for useful discussions and to M. Gumin for comments on the original version of this paper. We thank the organizers and participants of spring 2012 ESI workshop on Higher Spin Gravity, where this work was initiated, for creation of friendly and productive atmosphere and stimulating conversations. Also we thank the Galileo Galilei Institute for Theoretical Physics for the hospitality and the INFN for partial support during the 
final stage of this work. This research was supported in part by RFBR Grant No 11-02-00814-a.

\section{References}

[1] C. Fronsdal, "Massless Particles, Ortosymplectic Symmetry and Another Type of KaluzaKlein Theory", Preprint UCLA/85/TEP/10, in Essays on Supersymmetry, Reidel, 1986 (Mathematical Physics Studies, v.8).

[2] I. Bandos and J. Lukierski, Mod. Phys. Lett. A 14 (1999) 1257, [ hep-th/9811022].

[3] I. A. Bandos, J. Lukierski and D. P. Sorokin, Phys. Rev. D 61 (2000) 045002 [arXiv:hepth/9904109].

[4] M. A. Vasiliev, Phys. Rev. D 66 (2002) 066006 [hep-th/0106149].

[5] M. A. Vasiliev," Relativity, Causality, Locality, Quantization and Duality in the $S p(2 M)$ Invariant Generalized Space-Time", Contribution to the Marinov's Memorial Volume, M.Olshanetsky and A.Vainshtein Eds, World Scientific, [hep-th/0111119].

[6] I. Bandos, X. Bekaert, J. A. de Azcarraga, D. Sorokin and M. Tsulaia, JHEP 0505 (2005) 031 [arXiv:hep-th/0501113].

[7] H. Osborn and A. C. Petkou, Annals Phys. 231 (1994) 311 [hep-th/9307010].

[8] A. C. Petkou, JHEP 0303 (2003) 049 [hep-th/0302063].

[9] S. Giombi, S. Prakash and X. Yin, JHEP 1307 (2013) 105 [arXiv:1104.4317 [hep-th]].

[10] M. S. Costa, J. Penedones, D. Poland and S. Rychkov, JHEP 1111 (2011) 071 [arXiv:1107.3554 [hep-th]].

[11] S. Giombi and X. Yin, J. Phys. A 46 (2013) 214003 [arXiv:1208.4036 [hep-th]].

[12] Y. S. Stanev, Nucl. Phys. B 865 (2012) 200 [arXiv:1206.5639 [hep-th]].

[13] A. Zhiboedov, arXiv:1206.6370 [hep-th].

[14] N. Colombo and P. Sundell, arXiv:1208.3880 [hep-th].

[15] V. E. Didenko and E. D. Skvortsov, arXiv:1210.7963 [hep-th].

[16] M. A. Vasiliev, Class. Quant. Grav. 30 (2013) 104006 [arXiv:1212.6071 [hep-th]].

[17] O. A. Gelfond and M. A. Vasiliev, Theor.Math.Phys. 145 (2005) 35 [ hep-th/0304020].

[18] N. Colombo and P. Sundell, JHEP 1111 (2011) 042 [arXiv:1012.0813 [hep-th]].

[19] I. R. Klebanov and A. M. Polyakov, Phys. Lett. B 550 (2002) 213 [arXiv:hep-th/0210114].

[20] M. A. Vasiliev, Phys. Lett. B 285 (1992) 225.

[21] E. Sezgin and P. Sundell, JHEP 0507 (2005) 044 [arXiv:hep-th/0305040]. 
[22] J. Maldacena and A. Zhiboedov, J. Phys. A 46 (2013) 214011 [arXiv:1112.1016 [hep-th]].

[23] J. Maldacena and A. Zhiboedov, Class.Quant.Grav. 30 (2013), arXiv:1204.3882 [hep-th].

[24] M. A. Vasiliev, J. Phys. A 46 (2013) 214013 [arXiv:1203.5554 [hep-th]].

[25] O. V. Shaynkman and M. A. Vasiliev, Theor. Math. Phys. 128 (2001) 1155 [Teor. Mat. Fiz. 128 (2001) 378] [hep-th/0103208].

[26] M. A. Vasiliev, Ann. Phys. (NY) 190 (1989) 59.

[27] X. Bekaert, S. Cnockaert, C. Iazeolla and M. A. Vasiliev, hep-th/0503128.

[28] O. A. Gelfond and M. A. Vasiliev, JHEP 03 (2009) 125; [arXiv:0801.2191v4 [hep-th]].

[29] C. L. Siegel, "Sympletic Geometry", Academic Press, 1964.

[30] D. Mumford, "Tata lectures on theta", Boston-Basel-Stuttgart: Birkhäuser, vol. I, 1983, vol. II, 1984.

[31] R. G. Leigh and A. C. Petkou, arXiv:1212.4421 [hep-th].

[32] A. Jevicki, K. Jin and Q. Ye, J. Phys. A 46 (2013) 214005 [arXiv:1212.5215 [hep-th]].

[33] N. M. Nikolov, Y. S. Stanev and I. T. Todorov, J. Phys. A 35 (2002) 2985 [hep-th/0110230].

[34] O. A. Gelfond, M. A. Vasiliev, arXiv:1012.3143 [hep-th]

[35] M. A. Vasiliev, Russ. Phys. J. 45 (2002) 670 (Izv. Vuzov, Fizica 45 (2002) N7 23), [hepth/0204167].

[36] M. A. Vasiliev, Fortsch. Phys. 36 (1988) 33.

[37] E. S. Fradkin and M. A. Vasiliev, Int. J. Mod. Phys. A 3 (1988) 2983.

[38] S. E. Konstein and M. A. Vasiliev, Nucl. Phys. B 331 (1990) 475.

[39] F.A. Berezin, M.A. Shubin, "Schrödinger Equation", Moscow Univercity Edition, 1983.

[40] V. E. Didenko and M. A. Vasiliev, Phys. Lett. B 682 (2009) 305 [arXiv:0906.3898 [hep-th]].

[41] S. F. Prokushkin and M. A. Vasiliev, Nucl. Phys. B 545 (1999) 385 [hep-th/9806236].

[42] O. A. Gelfond, and M. A. Vasiliev, to appear.

[43] M. R. Gaberdiel and R. Gopakumar, J. Phys. A 46 (2013) 214002 [arXiv:1207.6697 [hepth]].

[44] N. Arkani-Hamed, J. L. Bourjaily, F. Cachazo, A. B. Goncharov, A. Postnikov and J. Trnka, arXiv:1212.5605 [hep-th].

[45] M. A. Vasiliev, Int. J. Geom. Meth. Mod. Phys. 3 (2006) 37 [hep-th/0504090]. 